\title{
Review
}

\section{The Pancreatic $\beta$-Cell: The Perfect Redox System}

\author{
Petr Ježek *(1), Blanka Holendová (D), Martin Jabůrek (D), Jan Tauber, Andrea Dlasková and Lydie Plecitá-Hlavatá \\ Department of Mitochondrial Physiology, No.75, Institute of Physiology of the Czech Academy of Sciences, \\ 14220 Prague, Czech Republic; blanka.holendova@fgu.cas.cz (B.H.); martin.jaburek@fgu.cas.cz (M.J.); \\ jan.tauber@fgu.cas.cz (J.T.); andrea.dlaskova@fgu.cas.cz (A.D.); lydie.plecita@fgu.cas.cz (L.P.-H.) \\ * Correspondence: jezek@biomed.cas.cz; Tel.: +420-296442760
}

Citation: Ježek, P.; Holendová, B.; Jabůrek, M.; Tauber, J.; Dlasková, A.; Plecitá-Hlavatá, L. The Pancreatic $\beta$-Cell: The Perfect Redox System. Antioxidants 2021, 10, 197. https://doi.org/10.3390/ antiox10020197

Academic Editor: Saverio Francesco Retta

Received: 29 December 2020

Accepted: 25 January 2021

Published: 29 January 2021

Publisher's Note: MDPI stays neutral with regard to jurisdictional claims in published maps and institutional affiliations.

\section{Copyright: (c) 2021 by the authors.} Licensee MDPI, Basel, Switzerland. This article is an open access article distributed under the terms and conditions of the Creative Commons Attribution (CC BY) license (https:// creativecommons.org/licenses/by/ $4.0 /)$.

\begin{abstract}
Pancreatic $\beta$-cell insulin secretion, which responds to various secretagogues and hormonal regulations, is reviewed here, emphasizing the fundamental redox signaling by NADPH oxidase 4- (NOX4-) mediated $\mathrm{H}_{2} \mathrm{O}_{2}$ production for glucose-stimulated insulin secretion (GSIS). There is a logical summation that integrates both metabolic plus redox homeostasis because the ATP-sensitive $\mathrm{K}^{+}$channel ( $\mathrm{K}_{\text {ATP }}$ ) can only be closed when both ATP and $\mathrm{H}_{2} \mathrm{O}_{2}$ are elevated. Otherwise ATP would block $\mathrm{K}_{\text {ATP, }}$, while $\mathrm{H}_{2} \mathrm{O}_{2}$ would activate any of the redox-sensitive nonspecific calcium channels (NSCCs), such as TRPM2. Notably, a $100 \%$-closed $\mathrm{K}_{\text {ATP }}$ ensemble is insufficient to reach the $-50 \mathrm{mV}$ threshold plasma membrane depolarization required for the activation of voltage-dependent $\mathrm{Ca}^{2+}$ channels. Open synergic NSCCs or $\mathrm{Cl}^{-}$channels have to act simultaneously to reach this threshold. The resulting intermittent cytosolic $\mathrm{Ca}^{2+}$-increases lead to the pulsatile exocytosis of insulin granule vesicles (IGVs). The incretin (e.g., GLP-1) amplification of GSIS stems from receptor signaling leading to activating the phosphorylation of TRPM channels and effects on other channels to intensify integral $\mathrm{Ca}^{2+}$-influx (fortified by endoplasmic reticulum $\mathrm{Ca}^{2+}$ ). ATP plus $\mathrm{H}_{2} \mathrm{O}_{2}$ are also required for branched-chain ketoacids (BCKAs); and partly for fatty acids (FAs) to secrete insulin, while BCKA or FA $\beta$-oxidation provide redox signaling from mitochondria, which proceeds by $\mathrm{H}_{2} \mathrm{O}_{2}$ diffusion or hypothetical SH relay via peroxiredoxin "redox kiss" to target proteins.
\end{abstract}

Keywords: pancreatic $\beta$-cells; insulin secretion; redox signaling; NADPH oxidase 4; branched-chain ketoacid oxidation; fatty-acid-stimulated insulin secretion; ATP-sensitive $\mathrm{K}^{+}$channel; TRPM channels; GLP-1; GPR40

\section{Introduction \\ 1.1. Emerging Concept of Redox Signaling}

The emerging concept of redox signaling resulted from ceaseless efforts, and decades of studies of the redox homeostasis of cells and cell organelles. Among organelles, the mitochondrion combines the key roles of the metabolic and redox hub. Prototypical redox signaling is involved in mechanisms of hypoxia-inducible factor (HIF) system-initiated transcriptome reprogramming. Both cytosolic and mitochondrial redox signaling were implicated, e.g., in HIF-1 $\alpha$ stabilization, either via the oxidation of iron II to iron III of the reaction center of prolyl hydroxylase domain enzymes (PHD/EglN) [1,2] and/or their sensitive cysteines [3].

Recently, we revealed another physiological redox signaling of enormous biomedical significance: NADPH oxidase 4- (NOX4-) initiated redox signaling $\left(\mathrm{H}_{2} \mathrm{O}_{2}\right)$, which fundamentally determines the 1st phase of glucose-stimulated insulin secretion (GSIS) in pancreatic $\beta$-cells, hypothetically by a cooperative closing of the ATP-sensitive $\mathrm{K}^{+}$channel $\left(\mathrm{K}_{\mathrm{ATP}}\right)$ together with ATP elevation (Figure 1A) [4]; or alternatively (Figure 1B), we may assume that the essentially synergic channels, such as transient receptor potential melastin (TRPM) isoform 2, i.e., TRPM2, are also activated by $\mathrm{H}_{2} \mathrm{O}_{2}$ as reported [5], when the $100 \%$ $\mathrm{K}_{\mathrm{ATP}}$ ensemble is closed by the elevated ATP. 


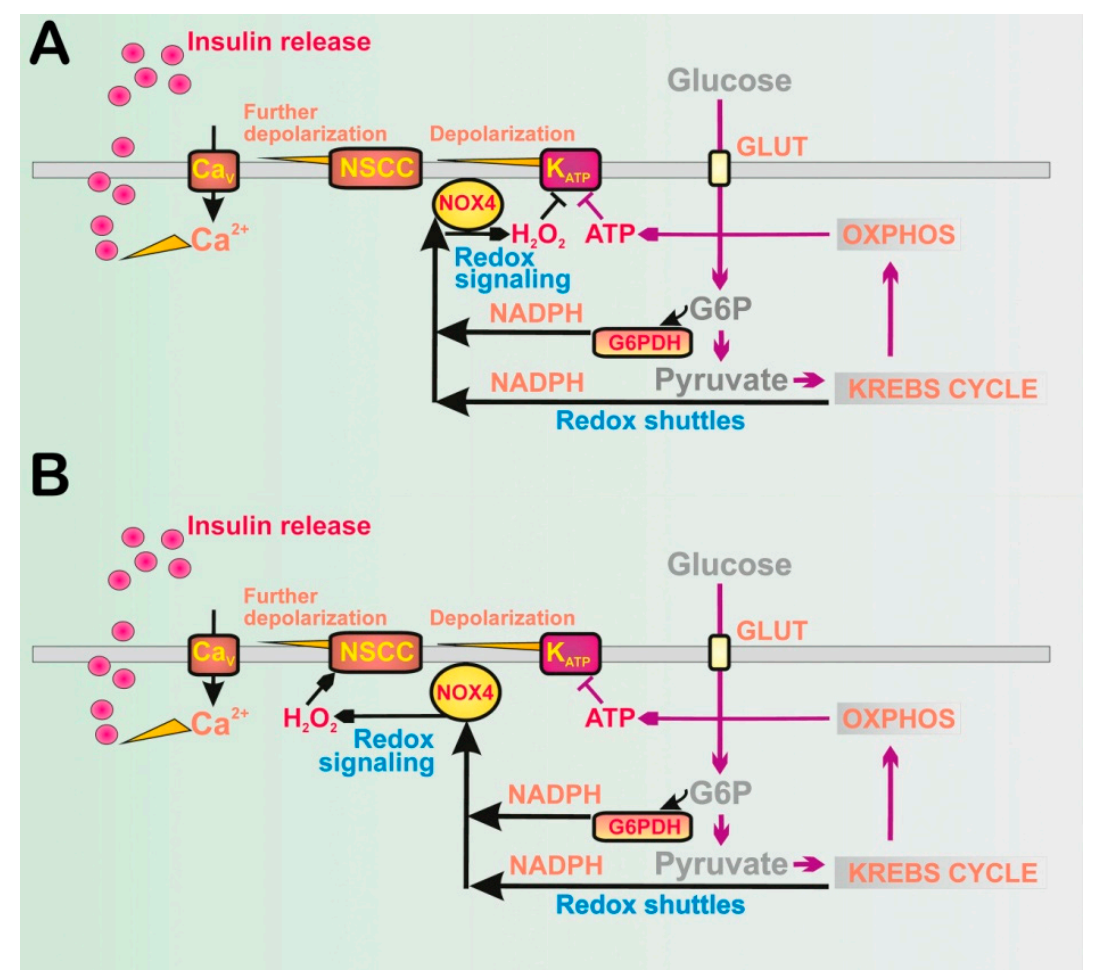

Figure 1. A new paradigm in the mechanism of glucose-stimulated insulin secretion (GSIS): Essential requirement of redox signaling. Upon glucose intake, pentose phosphate pathway (PPP) and redox shuttles supply cytosolic NADPH to increase NOX4 activity and thus elevate $\mathrm{H}_{2} \mathrm{O}_{2}$, which substantiates redox signaling. The two possible hypotheses assume two distinct targets of $\mathrm{H}_{2} \mathrm{O}_{2}$ : either that (A) $\mathrm{K}_{\text {ATP }}$ is closed exclusively when both ATP plus $\mathrm{H}_{2} \mathrm{O}_{2}$ are elevated (as supported by the patch-clamp data in Ref. [4]); or (B) that $\mathrm{K}_{\text {ATP }}$ is closed by ATP, whereas the TRPM2 channel (or other nonspecific calcium channels (NSCCs)) is activated by $\mathrm{H}_{2} \mathrm{O}_{2}$. In both cases, the depolarization shift by NSCCs is essentially required for achieving the $-50 \mathrm{mV}$ depolarization threshold for $\mathrm{Ca}_{\mathrm{V}}$ activation despite $100 \%$ of the $\mathrm{K}_{\mathrm{ATP}}$ ensemble being closed. Mechanism (A) plus additional $\mathrm{H}_{2} \mathrm{O}_{2}$ stimulation of TRPM2 is also plausible.

The inability of the closure of the entire $\mathrm{K}_{\mathrm{ATP}}$ ensemble to reach the threshold depolarization of the plasma membrane for opening voltage-gated $\mathrm{Ca}^{2+}$ channels $\left(\mathrm{Ca}_{\mathrm{V}}\right)[6]$ is discussed in this review as the key element for understanding the mechanism of GSIS. Thus synergy is required with the other channels. Moreover, the principle of a "logical summation" of metabolic plus redox stimulation will be discussed and the experimental evidence supporting it. This principle seems to be universal for any mechanism of insulin secretion dependent on $\mathrm{K}_{\mathrm{ATP}}$ and/or $\mathrm{Ca}_{\mathrm{V}}$.

For example the alternative, in this case mitochondrial redox signaling, is essentially required for insulin secretion stimulated by metabolites of branched-chain amino acids (BCAAs), i.e., BC keto acids (BCKAs), including 2-ketoisocaproate (KIC; termed also 2oxoisocaproate, OIC; leucine metabolite), 2-ketoisovalerate (KIV; valine metabolite) and 2-ketomethylvalerate (KMV; isoleucine metabolite). Evidence for this mechanism stems from the effects of mitochondrial matrix-targeted antioxidant SkQ1, which does not affect GSIS in INS-1E cells, but completely inhibits insulin secretion stimulated by BCKAs, e.g., by KIC [4]. The interpretation of these results is that the NOX4 source of $\mathrm{H}_{2} \mathrm{O}_{2}$ cannot be easily inhibited by SkQ1 located within the inner phospholipid leaflet of the inner mitochondrial membrane (IMM), whereas the redox signaling originating from the mitochondrion must be blocked.

Previously, also coenzyme A-esters (CoA-esters) of fatty acids (FAs), malonyl-CoA and long-chain acyl-CoA were suggested to be coupling factors for nutrient stimulus-insulin 
secretion coupling [7]. Current complex knowledge on BCKAs and FAs in relation to insulin secretion with an emphasis on redox signaling is also reviewed here.

It has been established that the content of glutathione is relatively low in pancreatic $\beta$-cells [8-11], in contrast to the content of thioredoxins and glutaredoxins [12,13], peroxiredoxins and other proteins capable of redox relay, hence suitable for conducting and spreading redox signals $[14,15]$. From this point of view, the pancreatic $\beta$-cell appears to be a perfect redox system and ideally suited for spreading the redox signal.

Without knowing all the details of this redox system and the sensing of glucose or other secretagogues, one cannot judge their impairment during the development of type 2 diabetes. However, discussion of the resulting pathology is outside the scope of this review article, and the origin of oxidative stress in diabetic pancreatic $\beta$-cells, which has been reviewed elsewhere [16]. Neither do we discuss the relations to and effects of redox signaling on gene expression in pancreatic $\beta$-cells. The repeatable redox signaling in pancreatic $\beta$-cells, such as during GSIS, was suggested to maintain the correct expression of the insulin gene (Ins) [17]. The repeatable redox signaling could hypothetically maintain the other transcription factors or proteins specific for the optimum fitness and identity of $\beta$-cells. This vast area of knowledge is beyond the scope of this review.

\subsection{Traditional Classification of Insulin Secretion Mechanisms}

The consensus of reports on GSIS has been that it involves two classes of mechanisms or pathways [18-26]. By definition, triggering mechanism(s) were exclusively ascribed to the canonical one or those involving the $\mathrm{K}_{\mathrm{ATP}}$-channel-dependent $\mathrm{Ca}^{2+}$ influx into $\beta$-cells provided by $\mathrm{Ca} v$ opening. In turn, the metabolic amplifying mechanisms were defined as those involving mechanisms for receptor signaling that activate insulin secretion, including incretin receptor and metabotropic receptor signaling. The latter typically involves other secretagogues, such as FAs. Certain inconsistencies in such a classification are apparent. For example, upon the stimulation of insulin secretion by FAs at low, non-stimulating glucose, there are "mixed" mechanisms or pathways, which are both $\mathrm{K}_{\mathrm{ATP}}$-channel dependent and receptor dependent. The latter is also dependent (but maybe only partially) on the $\mathrm{Ca}_{\mathrm{V}}$-mediated $\mathrm{Ca}^{2+}$ influx into $\beta$-cells. It is questionable whether there are any mechanisms that do not involve the primary $\mathrm{Ca}_{\mathrm{V}}$-mediated $\mathrm{Ca}^{2+}$ influx. Speculatively, such a net amplifying mechanism should be exclusively for the docking, fusion and exocytosis of insulin granule vesicles (IGVs) independent of $\mathrm{Ca}_{V}$-triggering. Hence, the responses of the exocytotic machinery should be given by the elevating cytosolic $\mathrm{Ca}^{2+}$ concentration $\left[\mathrm{Ca}^{2+}\right]_{\mathrm{c}}$, which is not triggered by the primary $\mathrm{Ca}_{\mathrm{V}}$-mediated $\mathrm{Ca}^{2+}$ influx (as for the $\mathrm{Ca}^{2+}$ efflux from ER), but originates from signaling by protein kinase A (PKA; see Section 2.5.2), by a pathway of enhanced signaling via exchange proteins directly activated by cAMP 2 (EPAC2; see Section 2.5.3) and or G-protein-coupled receptors (GPR; see Sections 2.5.5 and 6). Nevertheless, typically information signaling induces mixed responses involving both $\mathrm{Ca}_{\mathrm{V}}$-triggering plus the facilitation of IGV exocytosis. Even if we do not know whether these two mechanism categories can exist separately, we use the term "Cav-dependent" or " $\mathrm{Ca}_{\mathrm{V}}$ - plus receptor-dependent" insulin secretion, while the latter can be subclassified, whether it includes the $\mathrm{Ca}^{2+}$-efflux from the ER or not (Figure 2). The ER-Ca ${ }^{2+}$-independent mechanisms thus originate from the direct signaling of PKA, EPAC2 and GPR, exclusively affecting targets among the IGV-exocytotic machinery. 


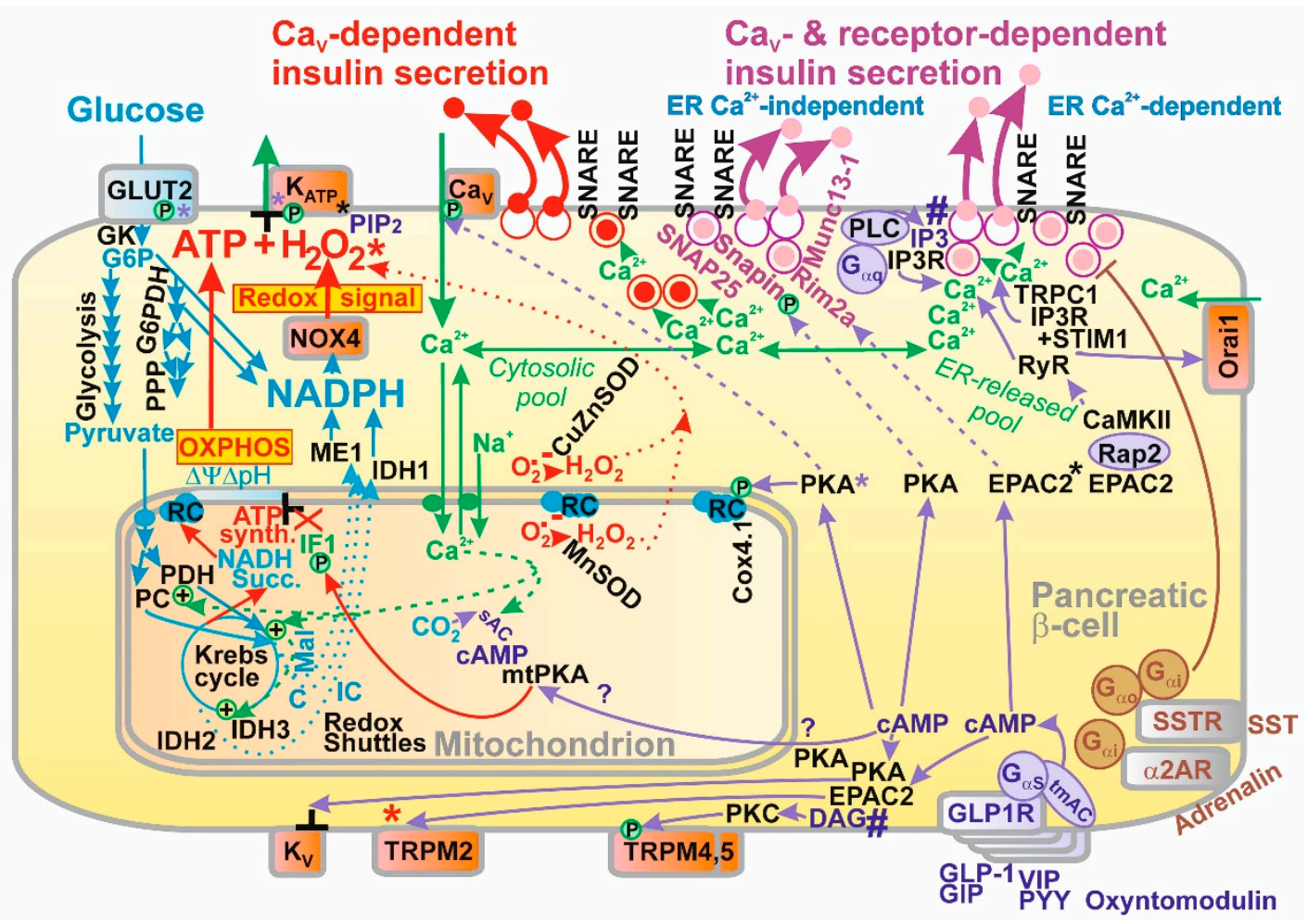

Figure 2. A new paradigm in the mechanism of GSIS in the context of the regulation of insulin secretion. Glucose transporters, GLUT2 in rodents and GLUT1 in humans, allow the equilibration of blood glucose with the cytosolic glucose concentration in pancreatic $\beta$-cells at any time. A specific hexokinase isoform, glucokinase (GK) is not feedback inhibited, which together with the absence of functional pyruvate dehydrogenase (PDH) kinases and lactate dehydrogenase, allows the entire glycolytic output to be utilized for oxidative phosphorylation (OXPHOS) and hence efficient synthesis of ATP. As a result, elevated blood glucose induces elevated ATP synthesis and respiratory chain (RC) proton pumping, thus increasing $\Delta \Psi_{\mathrm{m}}$ and respiration. Moreover, a portion of glucose-6-phosphate formed by GK enters the PPP, producing NADPH at two sites, by G6P dehydrogenase (G6PDH) and 6-phosphogluconate dehydrogenase. This elevates cytosolic NADPH and thus the NOX4 activity. Since NOX4 directly produces $\mathrm{H}_{2} \mathrm{O}_{2}$, elevated glucose evokes instant redox signaling. Hypothetically, either $\mathrm{K}_{\text {ATP }}$ is only closed when both ATP and $\mathrm{H}_{2} \mathrm{O}_{2}$ are elevated and TRPM2 can also be independently activated by $\mathrm{H}_{2} \mathrm{O}_{2}$ (red *); or ATP only closes $\mathrm{K}_{\text {ATP }}$ and $\mathrm{H}_{2} \mathrm{O}_{2}$ activates TRPM2, as one of NSCCs, which is essentially required to reach the threshold of $-50 \mathrm{mV}$ for the activation of $\mathrm{Ca}_{\mathrm{V}}$. The intermittent opening of $\mathrm{Ca}_{\mathrm{V}}$ channels followed by the opening of $\mathrm{K}_{\mathrm{V}}$ leads to repeatable action potential spikes, provoking pulsatile $\mathrm{Ca}^{2+}$ entry and oscillations in the cytosolic Ca ${ }^{2+}$ concentrations, which stimulate the exocytosis of insulin granules. There are amplifying regulation systems: (i) given by the $\mathrm{Ca}^{2+}$ import to the mitochondrial matrix, where increased $\mathrm{Ca}^{2+}$ activates several dehydrogenases, including the PDH complex; and activates the PKA-mediated phosphorylation of Cox4.1 and sAC-mtPKA-mediated phosphorylation of IF1, where the former protects the ATP inhibition of Cox4.1, and the latter releases the inhibition of the ATP-synthase and (ii) redox shuttles contribute to the elevated cytosolic NADPH. Incretins, GLP-1 or GIP, massively amplify insulin secretion via their receptors, transferring signals via the $\mathrm{G} \alpha$ s proteins, tmAC, cAMP and either the PKA or EPAC2A pathway. PKA increases the sensing range of $\mathrm{K}_{\mathrm{ATP}}$ to physiological $\mathrm{mM} \mathrm{ATP}$, inhibits $\mathrm{K}_{\mathrm{V}}$, activates $\mathrm{Ca}_{\mathrm{V}}$, GLUT2 (gray *) and snapin. EPAC2A directly activates TRPM2, essential for KATP-triggered GSIS; and activates Rim2 $\alpha$ or via Rap2-calmodulin kinase II (CaMKII) activates RyR, providing the Ca2+efflux from ER, when CaL is open, thus again amplifying IGV exocytosis. Biased GLP1R (GIPR) signaling activates PLC and either IP3-IP3R or PLC STIM1-Ora1-TRPC1 mediated Ca2+ efflux from the ER (or TRPC1 migration to the plasma membrane); or the DAG-PKC pathway phosphorylating and thus activating TRPM4 and 5, so providing another essential NSCC-mediated shift up to $-50 \mathrm{mV}$. Somatostatin (SST) and the andrenergic inhibitory pathway (via Gi or Go proteins) are also depicted.

Note also that the two phases of insulin secretion occur in experiments with mice or their PIs, even for FAs [27]. As such, each phase could be based on its own distinct spectrum of mechanisms, being amplified "separately" and by a slightly distinct mechanism. That is 
why in this review, we instead describe and discuss insulin secretion according to the primary secretagogue.

\section{Revisited Mechanism of Glucose-Stimulated Insulin Secretion}

2.1. Elevations of ATP Plus $\mathrm{H}_{2} \mathrm{O}_{2}$ as the Fundamental Condition for GSIS

\subsubsection{Discovery of NOX4-Mediated Redox Signaling in Pancreatic $\beta$-Cells}

Reactive oxygen species (ROS) have been implicated in insulin secretion. For example, mitochondrial ROS have been suggested to modulate insulin secretion [28], when resulting from mono-oleoyl-glycerol addition [29]. In Section 6 below, this is explained by the direct activation of GPR119 and possible fatty-acid- (FA-) stimulated insulin secretion (FASIS), while the observed ROS could originate from FA $\beta$-oxidation. Inhibition of the pentose phosphate pathway (PPP), which diminishes insulin secretion, was also reported to shift redox homeostasis [30]. Instead an unspecified link of GSIS with the externally added $\mathrm{H}_{2} \mathrm{O}_{2}$ was observed, and the effects of antioxidants at decreased glutathione by diethylmaleate in INS-1(823/13) cells [31]. The inhibition of an unidentified isoform of NADPH oxidase was also reported to attenuate GSIS when $\beta$-cells were treated with an antisense p47PHOX oligonucleotide [32] or with a nonspecific inhibitor of NADPH oxidase (DPI), which also inhibits complex I of the mitochondrial respiratory chain (RC) [33,34]. Another report employed an inhibitor of the two specific isoforms of NADPH oxidases (NOXs) [29].

Nevertheless, it was not known which NOX isoform actually participates in GSIS, or if any is involved at all. Thus, the traditional consensus on a "textbook" GSIS mechanism was maintained, which considers the ATP elevation (or elevation of the ATP/ADP ratio) to be the only requirement and a sufficient condition for triggering insulin secretion (e.g., by the $\mathrm{K}_{\text {ATP }}$ closing), with no need for any parallel redox signaling [18-21]. It had never been considered that the ATP increase by itself is insufficient for GSIS. In contrast, we demonstrated that the elevated oxidative phosphorylation (OXPHOS), leading to the increased ATP levels and ATP / ADP ratio at the peri-plasmamembrane space in the vicinity of $\mathrm{K}_{\mathrm{ATP}}$, is insufficient to initiate GSIS [4]. Additionally, it had never been considered that any redox signaling might essentially participate in GSIS.

We have finally demonstrated that NADPH oxidase isoform 4 (NOX4) is fundamentally required for GSIS [4]. In model rat pancreatic $\beta$-cells (INS-1E cells) with silenced NOX4 or in full NOX4 knockout (NOX4KO) mice and in mice with NOX4 knockout specifically in pancreatic $\beta$-cells (NOX4 $\beta \mathrm{KO}$ mice), the 1st phase of GSIS was largely blocked [4]. Unlike in INS-1E cells (where two phases of insulin secretion do not exist), in both NOX4 KO mice strains and in their isolated pancreatic islets (PIs), the 1st (fast) phase of GSIS was abolished with NOX4 ablation. In PIs, either the overexpression of NOX4 (achieved at least in the peripheral spheroid layer of islets) or additions of $\mathrm{H}_{2} \mathrm{O}_{2}$ rescued this 1st fast phase of GSIS. No effects were found in NOX2 KO mice, despite NOX2 having been previously implicated to play an antagonistic role in redox homeostasis [35]. In turn, similar effects were observed with doxycycline-inducible $\beta$-cell-specific NOX5 knockout mice [36].

Moreover, using the patch-clamp of INS-1E cells silenced for NOX4, no glucoseinduced closure of a channel with all the characteristics of $K_{\text {ATP }}$ was observed [4]. Simultaneously, in INS-1E cells transfected with scrambled siRNA, we confirmed the well-known closure of $\mathrm{K}_{\mathrm{ATP}}$, as induced by glucose. These experiments supported the model in which $\mathrm{K}_{\mathrm{ATP}}$ (not together with the essentially synergic channels, such as TRPM2) integrates metabolic and redox homeostasis, and the channel system acts as a logical summation for which both elevated ATP plus elevated $\mathrm{H}_{2} \mathrm{O}_{2}$ exclusively lead to the required plasma membrane depolarization in order to activate $\mathrm{Ca}_{\mathrm{V}}$. Elevations of either ATP alone or $\mathrm{H}_{2} \mathrm{O}_{2}$ alone cannot establish such conditions (Figures 1 and 2). The current most plausible hypotheses are that either both elevated ATP plus $\mathrm{H}_{2} \mathrm{O}_{2}$ act directly on $\mathrm{K}_{\text {ATP }}$ (Figure $1 \mathrm{~A}$ ), or alternatively the TRPM2- $\mathrm{K}_{\text {ATP }}$ synergy could be behind this effect as well, in which ATP would close $\mathrm{K}_{\text {ATP }}$ and $\mathrm{H}_{2} \mathrm{O}_{2}$ would active TRPM2 or other nonspecific calcium channels (NSCCs) [5], termed here as the "essentially synergic" channels (Figure 1B). Of course, both these mechanisms could also be in effect. 
We also documented that the redox signaling upon GSIS is provided by elevations of cytosolic $\mathrm{H}_{2} \mathrm{O}_{2}$, whereas ROS in the mitochondrial matrix (both $\mathrm{H}_{2} \mathrm{O}_{2}$ and superoxide release) are diminished due to the enhanced operation of the redox shuttles upon GSIS [37] (see Section 4). Since the silencing of glucose-6-phosphate (G6P) dehydrogenase (G6PDH) in INS-1E cells abolished approximately half of the GSIS rate [4], one may conclude that a portion of cytosolic NADPH as the substrate for NOX4 is provided by G6PDH and downstream 6-phosphogluconate dehydrogenase within the PPP, while the second portion is generated due to the operation of redox shuttles, i.e., due to NADPH increasingly produced by isocitrate dehydrogenate 1 (IDH1) and malic enzyme 1 (ME1) in the cytosol upon glucose intake [37]. The essential role of PPP was emphasized elsewhere [38].

As a result, we can draw schematics for the revisited mechanism of the 1st GSIS phase (Figures 1 and 2), while amplifying mechanisms based on the GLP-1 receptor (GLP1R) are also depicted in Figure 2. Elevated glucose metabolism, and specifically glycolysis, leads to an increased branching of the metabolic flux, particularly of G6P, toward PPP. Its enzymes, G6PDH and 6-phosphogluconate dehydrogenase, provide one of the NADPH sources for NOX4. PPP exhibits a rather low activity at low glucose, hence provides lower NADPH. Amplification of the cytosolic NADPH is also given by IDH1 and ME1 due to the elevated operation of the three redox shuttles described in Section 4. Since NOX4 was determined to be the only NADPH oxidase producing $\mathrm{H}_{2} \mathrm{O}_{2}$ directly [39,40], its elevated activity must lead to a profound increase in $\mathrm{H}_{2} \mathrm{O}_{2}$ release into the cytosolic compartment of $\beta$-cells. We have experimentally demonstrated in INS-1E cells and isolated islets that this indeed takes place, and that the excessive $\mathrm{H}_{2} \mathrm{O}_{2}$ release vanishes with NOX4 ablation [4].

In summary, the elevated $\mathrm{H}_{2} \mathrm{O}_{2}$, together with the concomitantly elevated ATP from the enhanced OXPHOS, is the only way to stimulate insulin secretion in a way that responds to the elevated glucose levels. As we discuss in detail in the Section 2.3, either ATP plus $\mathrm{H}_{2} \mathrm{O}_{2}$ are required for closing $\mathrm{K}_{\text {ATP }}$ [4] (Figure 1A); or ATP closes $\mathrm{K}_{\text {ATP }}$ and $\mathrm{H}_{2} \mathrm{O}_{2}$ activates the essential synergic channels (Figure 1B), such as NSCCs [5] or $\mathrm{Cl}^{-}$channels [41]. These synergic channels shift the insufficient depolarization produced by the $100 \%$-closed $\mathrm{K}_{\mathrm{ATP}}$ population towards the depolarization of the plasma membrane over the approximately $-50 \mathrm{mV}$ threshold required for the opening of $\mathrm{Ca}_{\mathrm{V}}$ [6] (Figure 3). In the latter case, there is no requirement for $\mathrm{K}_{\mathrm{ATP}}$ to be redox-regulated, but it still could be.

$\mathrm{Ca}_{\mathrm{V}}$ activation initiates action potential spikes, the descent part of which is governed by the opening of voltage-sensitive $\mathrm{K}^{+}$channels $\left(\mathrm{K}_{\mathrm{V}}\right)$ (Section 2.3). The resulting pulsatile $\mathrm{Ca}^{2+}$ influx is the pacemaker for the created oscillations in cytosolic $\left[\mathrm{Ca}^{2+}\right]_{\mathrm{c}}$, which are the main effector for the complex machinery of IGV exocytosis. That is why IGV exocytosis is also pulsatile. This machinery is described below in Section 2.6 and in the Figure 2 legend.

Besides the 1st phase, there is also a 2nd phase of insulin secretion, given at least by the contribution of a distinct delayed kinetics of IGV release [42,43]. Nevertheless, since it has been recognized that the 2nd phase, which is manifested in pancreatic islets, does not exist in the $\beta$-cells isolated from islets [44-46], it was suggested to originate from the synchronization of electrical activity of the plasma membrane potential within the ensemble of cells in the islet, leading to synchronization of their cytosolic $\mathrm{Ca}^{2+}$ oscillations [47] and other events. A few percent of pacemaker-like $\beta$-cells are responsible for such synchronization in rodents $[48,49]$. These cells were termed hub cells. Nevertheless, the delayed kinetics of the IGV release plus intercellular synchronization act in parallel.

Mechanisms of both net GSIS 1st and 2nd phases were traditionally classified as triggering, since both involve $\mathrm{Ca}$ activation resulting in enhanced cytosolic $\left[\mathrm{Ca}^{2+}\right]_{\mathrm{C}}[49]$. Moreover, the incretin-induced amplification mechanisms (typically induced by GLP-1 and GIP) are superimposed onto the canonical net GSIS mechanism. Thus incretins account for a large portion of the response to glucose in vivo (see Section 3). Multiple responses of $\beta$ cells to incretins mediated by incretin receptors further involve the additional incremental elevation of cytosolic $\mathrm{Ca}^{2+}$, such as from ER stores and the net facilitation of insulin granule vesicle docking, priming and exocytosis itself $[23,26]$. 


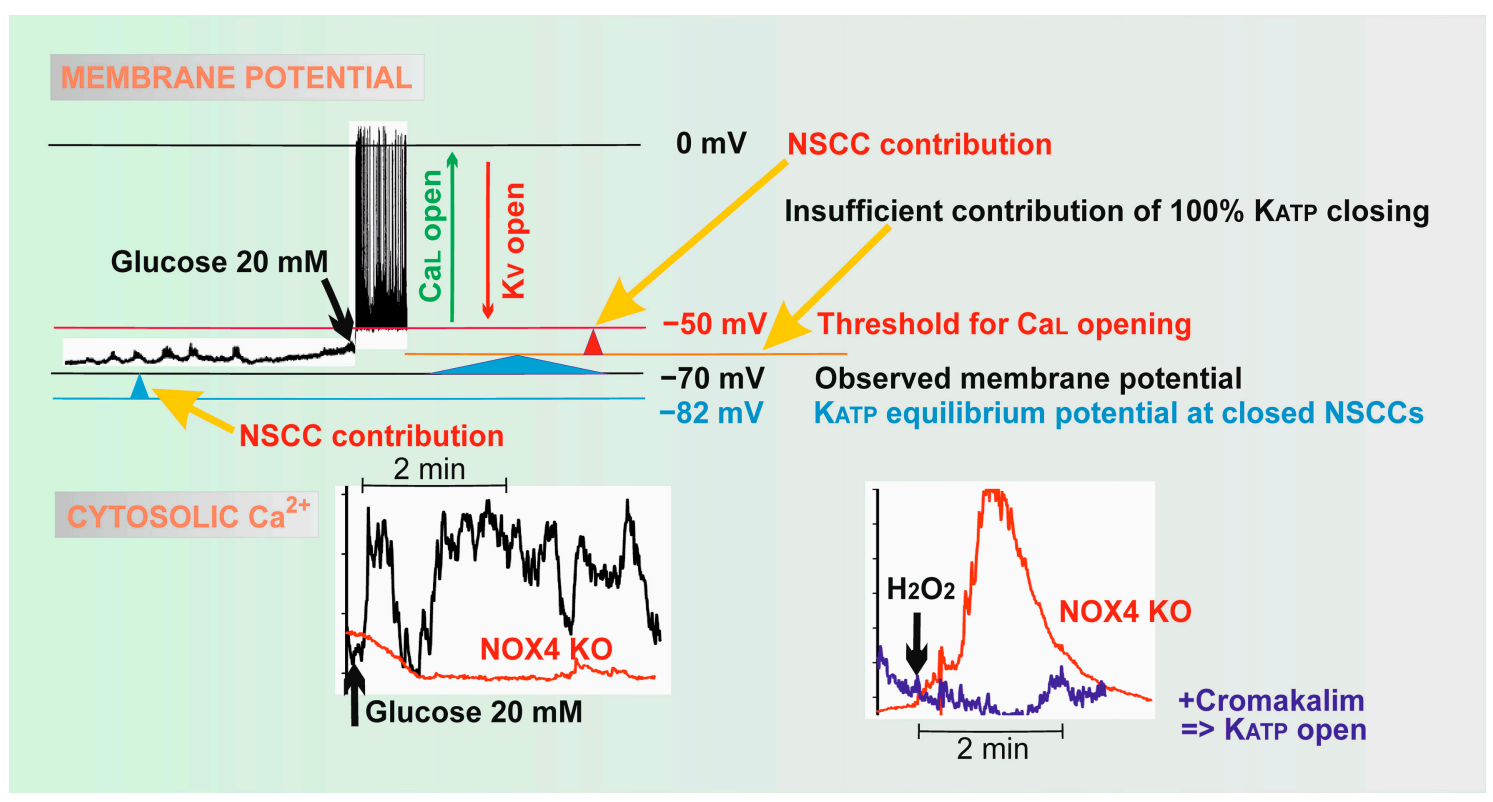

Figure 3. Schematics of parallel plasma membrane potential action potential vs. $\mathrm{Ca}^{2+}$ oscillations in pancreatic $\beta$-cells after the addition of $20 \mathrm{mM}$ glucose. Data similar to that published in Ref. [4] are used to illustrate: plasma membrane action potential firing (top) and $\mathrm{Ca}^{2+}$ oscillations indicated with FURA2 in INS-1E cells silenced for NOX4 (red trace "NOX4 KO") as compared with the $\mathrm{Ca}^{2+}$ oscillations rescued in silenced cells by $\mathrm{H}_{2} \mathrm{O}_{2}$ addition (bottom). The latter were prevented by cromakalim, keeping $\mathrm{K}_{\text {ATP }}$ open. The observed resting plasma membrane potential accounts for $-70 \mathrm{mV}$ in contrast to the theoretical potential of $-82 \mathrm{mV}$ at equilibrium (blue line), if $\mathrm{K}_{\mathrm{ATP}}$ was contributing exclusively. The shift is caused at least by the non-specific $\mathrm{Ca}^{2+}$ channels (NSCCs), the fluxes of which enable depolarization up to $-50 \mathrm{mV}$ (red line), above which the activation of $\mathrm{Ca}_{\mathrm{V}}$ channels proceeds. The intermittent $\mathrm{Ca}$ channel (and $\mathrm{Na}^{+}$channel) opening followed by the $\mathrm{K}_{\mathrm{V}}$ action creates the action potential spikes. Without the contribution of NSCCs, the $100 \%$ closed ensemble of $\mathrm{K}_{\text {ATP }}$ channels would only reach a depolarization (orange line) that is insufficient for the activation of $\mathrm{Ca}_{V}$. Since $\mathrm{Ca}^{2+}$ oscillations cease when $\mathrm{H}_{2} \mathrm{O}_{2}$ was not supplied by NOX4 (red trace), one can explain the essential requirement of redox signaling by the two possible above-mentioned hypotheses that differ in the two distinct targets of $\mathrm{H}_{2} \mathrm{O}_{2}$ : (A) either that $\mathrm{K}_{\text {ATP }}$ is closed exclusively when both ATP plus $\mathrm{H}_{2} \mathrm{O}_{2}$ are elevated (and TRPM2 can be independently also activated by $\mathrm{H}_{2} \mathrm{O}_{2}$ ); or $(\mathbf{B})$ that $\mathrm{K}_{\text {ATP }}$ is closed by ATP, whereas the TRPM2 channel (or other NSCCs) is activated by $\mathrm{H}_{2} \mathrm{O}_{2}$. In both cases, the depolarization shift by NSCCs is essentially required for achieving the $-50 \mathrm{mV}$ threshold for CaV activation, despite $100 \%$ of the $\mathrm{K}_{\mathrm{ATP}}$ ensemble being closed. Patch-clamp results tend to support model (A) [4].

\subsubsection{Glucose Equilibration and Specific Metabolism in Pancreatic $\beta$-Cells}

The consensus "canonical" mechanism of GSIS in the past has been an exclusive 100\% dependence on the elevated OXPHOS upon glucose intake in pancreatic $\beta$-cells. Indeed, pancreatic $\beta$-cells were adapted by phylogenesis to serve as a perfect glucose sensor. This is already enabled by the specific isoforms of glucose transporters, GLUT2 in rodents and GLUT1 in humans, which provide equilibration of the plasma glucose concentration with the cytosolic glucose concentration in $\beta$-cells [50,51]. Furthermore, a $\beta$-cell specific isoform IV of hexokinase (termed glucokinase) is not feedback inhibited by its product G6P, which enables efficient unidirectional flux [52,53].

Now we know that this G6P flux is branched into glycolysis and PPP [38]. The participation of PPP in GSIS has been previously questioned, since PPP was found to account for $10 \%$ of glucose utilization and G6PDH was found to be inhibited by increasing glucose $[54,55]$. Nevertheless, pioneering metabolomics studies have already identified PPP intermediates as being strongly associated with GSIS [56]. Inhibition of the 2nd NADPHproducing enzyme, 6-phosphogluconic acid dehydrogenase within PPP, by 6-amino nicotinamide was reported to suppress GSIS in INS-1E cells after a chronic exposure [57] or in rats [58]. The inefficiency of the acute action of 6-aminonicotinamide was also reported [56]. Additionally, the PPP enzyme transaldolase was reported to be activated by glucose [59]. 
Moreover, patients with a G6PDH deficiency had the first phase of insulin release impaired [60].

The near absence of lactate dehydrogenase in $\beta$-cells and the inefficiency of pyruvate dehydrogenase kinases (PDK, which otherwise would block pyruvate dehydrogenase, $\mathrm{PDH}$ ) then enables $100 \%$ of pyruvate and its equivalents (e.g., upon conversion by transaminases) to be utilized by OXPHOS. In pancreatic $\beta$-cells, PDK1 and PDK3 are "constitutively blocked" [61], and PDK2 does not phosphorylate the E1 $\alpha$ subunit of PDH, hence it does not inhibit its activity. As a result, at low basal glucose, the $\beta$-cell PDH exhibited a relatively high activity, which is only inhibited by $22 \%$ at high glucose. Moreover, the activity of the matrix-localized complexes of PDH and 2-oxoglutarate dehydrogenase and the activity of $\mathrm{NAD}^{+}$-dependent isocitrate dehydrogenase 3 (IDH3) are enhanced by the incoming $\mathrm{Ca}^{2+}$ to mitochondria upon GSIS [49]. A major cataplerosis is ensured by the dominant citrate efflux from the mitochondrial matrix [62].

The predominant pyruvate utilization proceeds via the mitochondrial matrix $\mathrm{PDH}-$ complex followed by the complete Krebs cycle. However, a minor pyruvate flux, providing oxaloacetate anaplerosis, is given by the pyruvate carboxylase [63]. Its reaction is important for the pyruvate/malate redox shuttle and phosphoenolpyruvate shuttle described below. With glutamine present, aminotransferases such as cytosolic ALT1 and mitochondrial ALT2 (also termed glutamate pyruvate transaminases, GPT1 and GPT2 [64]) could catalyze the reversible conversion of pyruvate plus L-glutamate to 2-oxoglutarate (2OG) and L-alanine [65] during glutaminolysis. Mitochondrial 2OG would enter back into the Krebs cycle. However, it was suggested that the glutamate dehydrogenase instead synthesizes glutamate, which subsequently facilitates the exocytosis of insulin granules $[66,67]$. If this is the case, the reaction would act in the reverse direction, producing L-glutamate and contributing to the pyruvate pool.

\subsubsection{Two Phases of GSIS}

There are two phases for the secretion of insulin responding to glucose in vivo [22,68,69], including GSIS in humans [70,71]. These two phases persist in isolated PIs. The $\mathrm{K}_{\mathrm{ATP}}$ dependent mechanism (termed also "triggering") and $\mathrm{K}_{\mathrm{ATP}}$-independent mechanisms contribute to both phases [23]. Note that the $\mathrm{K}_{\mathrm{ATP}}$-independent mechanisms still require the elevation of cytosolic $\mathrm{Ca}^{2+}$ [72]. Notably, it has been considered that the 2nd phase in vivo is independent of the extracellular glucose concentrations [73]. Mathematical models suggested that the 2nd phase of GSIS can be explained by the increased mobilization and priming of IGVs [74]. Thus also the cytosolic $\mathrm{Ca}^{2+}$ dynamics, which are even more complex, must contribute to the two phases. The issue of connectivity between $\beta$-cells within the pancreatic islets may also essentially contribute to the 2nd phase in rodents [45], but the mechanisms of how this is related to the delayed IGV kinetics are still to be elucidated. Additionally, note that human islets are morphologically different, and the connectivity issue has less importance, specifically for a high proportion of $\beta$-cell mass that is spread throughout the exocrine pancreas [75].

The first rapid and robust spike of insulin secretion occurs between 5 and $10 \mathrm{~min}$ after a bolus of glucose is administered in vivo or added to the isolated PIs. The explanation for this 1st phase of GSIS was based on how fast a fraction of existing IGVs can be released. The 1st phase was considered to be due to the predocked juxtaposed IGVs, located no more than 100-200 nm from the internal surface of the plasma membrane [76,77], and due to the newcomer IGVs, which may arrive within $50 \mathrm{~ms}$ and are not required to be predocked $[78,79]$.

The 2nd phase was then suggested to depend on a functional recruitment of IGVs and mobilization of reserve IGVs, which, consequently with a delay, replace the previously instantly released pool. Additionally, the 2nd phase was previously thought to be realized by newcomer vesicles without priming at the plasma membrane, but delayed due to the passage through the filamentous actin (F-actin) cytoskeleton. F-actin was reported to be reorganized at the same time to allow the IGV passage [80-82]. Nevertheless, further research 
demonstrated that there is an amplification of insulin secretion, which is independent of microfilaments $[81,83,84]$. However, recently various cytoskeleton components have been demonstrated to play a more detailed and complex role in the IGV exocytosis [42,43], being more than just a barrier (see Section 2.6).

When experimentally induced by a $60 \mathrm{mM}\left[\mathrm{K}^{+}\right]$concentration to artificially depolarize the plasma membrane, the IGV exocytosis only affects those IGVs that are already docked to the plasma membrane, whereas glucose stimulation leads to a new recruitment of IGVs to the plasma membrane [85]. As a result, potentiation phenomena, collectively termed the amplification of insulin-secretion coupling, are involved in both phases of insulin secretion, but the "amplification" exhibits a delayed kinetics relatively to the "triggering", which is instantaneous.

The 2nd GSIS phase can even last over $1 \mathrm{hr}$. Therefore, typically a higher amount of insulin is released in this phase. Experimentally, it became common practice to simulate the 2nd phase, either when $\mathrm{K}_{\mathrm{ATP}}$ channels are kept open by diazoxide and $\mathrm{KCl}$ is added to depolarize the plasma membrane; or when glibenclamide (a sulfonylurea drug) permanently closes $\mathrm{K}_{\mathrm{ATP}}$ independently of ATP. Both artificial manipulations reach the $-50 \mathrm{mV}$ depolarization threshold, hence enabling $\mathrm{Cav}_{\mathrm{V}}$ opening and $\mathrm{Ca}^{2+}$ influx. Note, however, that the overall cell $\mathrm{Ca}^{2+}$ distribution between organelles such as mitochondria, ER and the cytosol is undoubtedly different (and so also the consequences for IGV exocytosis), when elevations of cytosolic $\mathrm{Ca}^{2+}$ originate from cell receptor (information) signaling. Note also that the role of glutamate was reconsidered, and instead of belonging to major factors influencing GSIS, glutamate is regarded as being essential for additional IGV exocytosis upon GSIS amplification by GLP-1 [67].

\subsubsection{Inconsistencies in Considering Exclusive GSIS Dependence on Elevated ATP in Pancreatic $\beta$-Cells}

Several inconsistencies can be found, e.g., in INS-1E cells, which cannot be completely explained when considering the previous model for the 1st phase of GSIS, relying exclusively on the elevated ATP. At first, upon a complete block of the ATP-synthase with oligomycin, the closure of $\mathrm{K}_{\mathrm{ATP}}$ is incomplete [4]. The ATP-synthase is the terminal OXPHOS enzyme, synthesizing ATP and releasing it into the mitochondrial matrix. Similarly, a predominant fraction of vestigial ATP-synthases with the missing subunit DAPIT in INS-1E cells enable GSIS, despite elevations of ATP in these cells being only $10 \%$ of those in non-transgenic cells [86]. These data indirectly support the requirement of $\mathrm{H}_{2} \mathrm{O}_{2}$.

Additionally, there is now an alternative interpretation of the reported experiments with long-chain acyl-CoAs. Long-chain acyl-CoAs were reported to bind to the Kir6.2 subunit of $\mathrm{K}_{\mathrm{ATP}}$ [87], and therefore potently activate this channel $[88,89]$. Since GSIS was also found to be accompanied by a reduction in total cell acyl-CoAs and malonylCoA [62,90], it was hypothesized that this reduction facilitates $K_{\text {ATP }}$ closure [62]. Nevertheless, an alternative explanation would be that $\beta$-oxidation contributing to the acyl-CoA decrease provides the redox signaling toward $\mathrm{K}_{\mathrm{ATP}}$ and/or TRPM2, similarly as was described for KIC [4].

\subsection{Ion Channels Participating in GSIS}

\subsubsection{Plasma Membrane Potential and Ion Channels of Pancreatic $\beta$-Cells}

The plasma membrane of the $\beta$-cell contains up to 60 channels of 16 ion channel families [91]. Several channels are also located on the membrane of IGVs. In general, a distinct pattern of channels exists in different species, various cultured model $\beta$-cells or even within individual cells of PIs [75]. The resting plasma membrane potential $\left(V \mathrm{p}^{\mathrm{R}}\right)$ is given by a greater concentration of $\mathrm{K}^{+}$inside the $\beta$-cell $(150 \mathrm{mM})$ than outside $(5 \mathrm{mM})$ and predominantly by the activity of $\mathrm{K}^{+}$-channels. Hence, the plasma membrane permeability for $\mathrm{K}^{+}$is higher than for other ions. The actually measured $V \mathrm{p}^{\mathrm{R}}$ is $-75 \mathrm{mV}$ [92]. The closure of $100 \%$ of the $\mathrm{K}_{\text {ATP }}$ ensemble [91,93-95] was reported to lead to only an insufficient partial depolarization of the plasma membrane $[6,91]$. If the other channels did not contribute, 
the depolarization by the $100 \%$-closed $\mathrm{K}_{\text {ATP }}$ ensemble would not be sufficient to activate $\mathrm{Ca}_{\mathrm{V}}$ channels [96]. Thus, those "essential synergic" ion channels, notably redox-activated NSCCs, such as TRPM2, redox-activated NSCCs of other families [5], or even certain $\mathrm{Cl}^{-}$ channels [41], provide this essential enhancement of depolarization.

The resulting $\mathrm{Ca}^{2+}$ entry elevates the subplasmalemmal and cytosolic $\mathrm{Ca}^{2+}$ concentration and stimulates the $\mathrm{Ca}^{2+}$-dependent exocytosis of IGVs. However, $\mathrm{Ca}_{\mathrm{V}}$ opening is intermittent with the opening of the voltage-dependent $\left(\mathrm{K}_{\mathrm{V}}\right)$ channels [97] or calciumdependent $\mathrm{K}^{+}$-channels $\left(\mathrm{K}_{\mathrm{Ca}}\right)$ in humans. Thus $\mathrm{K}_{\mathrm{V}}$ or $\mathrm{K}_{\mathrm{Ca}}$ terminate $\mathrm{Ca}^{2+}$ entry. A simplified view describes that the single cycle of $\mathrm{Ca}_{\mathrm{V}}$ opening followed by $\mathrm{K}_{\mathrm{V}}$ opening determines each spike of the action potential firing. However, $\mathrm{Na}^{+}$-channels also help with creating upstrokes in $30 \%$ of the $\beta$-cell population [98]. The spike is repeated when $\mathrm{K}_{\mathrm{ATP}}$ together with the essential synergy channels still maintain sufficient depolarization. When gaps exist between a series of spikes, such as at $10 \mathrm{mM}$ glucose, a transient ATP consumption is responsible via sarco/ER-Ca ${ }^{2+}$-ATPase (SERCA) and plasmalemmal-Ca ${ }^{2+}$-ATPase (PMCA), i.e., ATPases removing $\mathrm{Ca}^{2+}$ [99]. In contrast, the involvement of ATP from cytosolic pyruvate kinases was indicated to add to the existing ATP pool during silent phases [100].

As for the fraction of $\mathrm{K}_{\mathrm{ATP}}$ channels, the action potential firing is not induced until $>93 \%$ of $K_{\text {ATP }}$ channels are closed [101]. In human $\beta$-cells, this fraction is even higher [101]. So, in mice, the closure of the remaining $7 \% \mathrm{~K}_{\mathrm{ATP}}$-population leads to the depolarization of the plasma membrane [102] in synergy with NSCCs or $\mathrm{Cl}^{-}$channels. This is because the activity of the whole $\mathrm{K}_{\mathrm{ATP}}$ channel population decreases almost exponentially with the increasing glucose concentration, the respective $\mathrm{K}_{\text {ATP }}$ current being already down to $50 \%$ at 2-3 mM glucose, and only $3 \%$ at $10 \mathrm{mM}$ glucose [103]. The $V \mathrm{p}$ remains stable at $2-3 \mathrm{mM}$ glucose. At about $7 \mathrm{mM}$ glucose, the $\mathrm{K}_{\mathrm{ATP}}$ channel current is so low that together with "synergic" channels, the attained depolarization leads to the action potential firing [91,92]. This situation is termed a supra-threshold depolarization. A nearly permanent firing exists at high $>25 \mathrm{mM}$ glucose [92]. Other channel phenomena cause the amplitude of permanent firing to become reduced by $15 \mathrm{mV}$ after $3 \mathrm{~min}$ [75].

The voltage-dependent $\mathrm{Ca}^{2+}$-channels $\mathrm{Ca}_{V}$ in mouse pancreatic $\beta$-cells are predominantly of the L-type $\left(\mathrm{Ca}_{\mathrm{L}}\right)$ with a minor population of R-, N- and P/Q-type [104,105]. $\mathrm{Ca}_{\mathrm{V}}$ channels contain four subunits, the pore forming the $\alpha 1$ subunit and auxiliary $\alpha 2$, $\beta$ and $\gamma$ subunit $[104,105]$. Several isoforms of the $\alpha 1$ subunit exist in pancreatic $\beta$-cells among species, such as $\mathrm{Ca}_{\mathrm{V}} 1.2, \mathrm{Ca}_{\mathrm{V}} 1.3, \mathrm{Ca}_{\mathrm{V}} 2.1, \mathrm{Ca}_{\mathrm{V}} 2.2, \mathrm{Ca}_{\mathrm{V}} 2.3, \mathrm{Ca}_{\mathrm{V}} 3.1$ and $\mathrm{Ca}_{\mathrm{V}} 3.2$, whereas the last two are found in human and rat $\beta$-cells [96]. They are responsible for different types of currents [104]. In mouse $\beta$-cells, Cav 1.2 and $\mathrm{Ca}_{V} 1.3$ are responsible for $50 \%$, $\mathrm{Ca}_{\mathrm{V}} 2.1$ for $15 \%$ and $\mathrm{Ca}_{\mathrm{V}} 2.3$ for $25 \%$ of the whole-cell $\mathrm{Ca}^{2+}$-current, which is activated at $-50 \mathrm{mV}$ [105]. Interestingly, Cav 2.3 contributes exclusively to the 2nd phase of GSIS [106]. The PKA-mediated phosphorylation of $\mathrm{Ca}_{\mathrm{V}} 1.2$ and $\mathrm{Ca}_{\mathrm{V}} 1.3$ enhances their activity [107].

\subsubsection{Detailed Sequence of Events in Plasma Membrane upon GSIS}

An intermediate depolarization at $10 \mathrm{mM}$ glucose was observed for mouse $\beta$-cells, which was reverted by a withdrawal of $\mathrm{Ca}^{2+}$ and $\mathrm{Na}^{+}$, evidencing the participation of other channels contributing to the depolarization (inward) flux, such as NSCCs [108]. Even an efflux of $\mathrm{Cl}^{-}$was suggested to fulfill this role [109], including the opening of LRRC8/VRAC anion channels $[41,110]$. Among NSCCs, participation was also suggested for members 4 (TRPM4) and 5 (TRPM5) of the TRPM cation channel subfamily [111]. TRPM2 is redox-activated via the oxidation of Met191 [5] and activated by nicotinic acid dinucleotide phosphate (NAADP) [112], which is elevated upon GSIS [113]. Interestingly, TRPM2 was also reported to interact with peroxiredoxin 2, from which it can receive the redox signal $[114,115]$. Additionally, $\mathrm{Ca}^{2+}$-activated TRPM4 and TRPM5 channels, heatactivated TRPV1 (capsaicin receptor), and TRPV2 or TRPV4 belong to the important group of NSCCs expressed in $\beta$-cells [6]. Among them, the transient receptor potential canonical (TRPC) member 3 (TRPC3) channel was suggested to induce the inward shift in Vp upon the activation of the GPR40 receptor [116]. 
As was noted above, the inward currents of certain levels by the opened NSCCs (or $\mathrm{Cl}^{-}$ channels) are essentially required for the induction of sufficient membrane depolarization in addition to the closing of $100 \%$ of the $\mathrm{K}_{\mathrm{ATP}}$ ensemble [6]. This is already reflected by the fact that the resting potential $V \mathrm{p}^{\mathrm{R}}$ of -75 to $-70 \mathrm{mV}$ is depolarized to some extent from the equilibrium $V \mathrm{p}^{\text {equi }}$ of $-82 \mathrm{mV}$ (calculated between 5 and $130 \mathrm{mM}\left[\mathrm{K}^{+}\right]$) (Figure 3). This shift is interpreted to exist due to NSCCs, since any $\mathrm{Na}^{+}, \mathrm{Ca}^{2+}$ and $\mathrm{K}^{+}$ ions can penetrate them [108]. Thus "synergic" channels provide a small background inward current that is unable to depolarize the plasma membrane at open $\mathrm{K}_{\mathrm{ATP}}$, but able to do so with the predominantly closed $\mathrm{K}_{\mathrm{ATP}}$-ensemble. The latter is possible since the NSCC-related conductance is then comparable to the small conductance provided by the remaining few \% of open $\mathrm{K}_{\mathrm{ATP}}$-channels (Figure 3 ). Without NSCCs, the established Vp would only be equal to $V \mathrm{p}^{\text {equi }}$, so any shift to $-50 \mathrm{mV}$ required for $\mathrm{Ca}_{\mathrm{V}}$ opening would not take place. As a result, the contribution by the basal opening of NSCCs is essential.

The same reasoning is also valid for anion channels, particularly $\mathrm{Cl}^{-}$channels. Active $\mathrm{Cl}^{-}$transport is provided in $\beta$-cells by SLC12A, SLC4A and SIC26A, which are able to set the cytosolic $\mathrm{Cl}^{-}$concentration above thermodynamic equilibrium. Besides the consideration of $\mathrm{GABA}_{A}, \mathrm{GABA}_{\mathrm{B}}$ and glycine receptor $\mathrm{Cl}^{-}$channels being involved in the insulin secretion machinery, also volume-regulated anion channels (VRACs) were considered to be open at high glucose [75]. VRACs are heteromers of leucine-rich repeat containing 8 isoform A (LRRC8A) with other LLRC8 isoforms, forming anion channels [41]. The ablation of LRRC8 in mice led to delayed $\mathrm{Ca}^{2+}$ responses of $\beta$-cells to glucose and diminished GSIS in mice, demonstrating the modulatory role of LRRC8A/VRAC on membrane depolarization leading to $\mathrm{Ca}_{\mathrm{L}}$ responses [110].

Intermittent $\mathrm{Ca}_{\mathrm{V}}$ opening leads to $V \mathrm{p}$ oscillations, which may be further influenced by the $\mathrm{Ca}^{2+}$ efflux from the ER [91,92]. In isolated mouse PIs, glucose was reported to induce cytosolic $\mathrm{Ca}^{2+}$ oscillations, which were superimposed from fast (2-60 s periods) and slow (up to several min) $\mathrm{Ca}^{2+}$ oscillations [117]. They originate from $V$ p oscillations of action potential firings and an interplay with $\mathrm{Ca}^{2+}$ efflux from ER [118], and evoke a pulsatile insulin secretion. The ER participation is provided by the phospholipase $C$ (PLC) of the plasma membrane, which responds to the glucose-stimulated $\mathrm{Ca}^{2+}$ influx. PLC produces inositol 1,4,5-triphosphate (IP3), which opens the $\mathrm{Ca}^{2+}$ channel of the IP3 receptor (IP3R) of ER. Another ER $\mathrm{Ca}^{2+}$ channel, the ryanodine receptor (RyR) may also participate, being activated by ATP, fructose, long-chain acyl-CoAs and cyclic adenosine 5 -diphosphate ribose [117]. Additionally, the role of other channels permitting storeoperated $\mathrm{Ca}^{2+}$ entry from the ER has been demonstrated, specifically the ternary complex of TRPC1/Orai1/STIM1 [6,119]. TRPC1 belongs to the TRPC family, which has a modest $\mathrm{Ca}^{2+}$ selectivity. TRPC1 interacts with Orai1 [120], and in such a functional complex, they are activated by STIM1 influx, the amplitude of $\mathrm{Ca}^{2+}$ oscillations and correlated with GSIS. The TRPC1 channels can be also recruited to the plasma membrane.

As was described above, the deactivation of $\mathrm{Ca}_{\mathrm{V}}$ is achieved by the opening of $\mathrm{K}_{\mathrm{V}}$ in rodents [97] or calcium-dependent $\left(\mathrm{K}_{\mathrm{Ca}}\right) \mathrm{K}^{+}$-channels in humans. Among the former, tetrameric $\mathrm{K}_{\mathrm{V}} 2.1$ is the prevalent form in rodent $\beta$-cells. A delayed rectifier $\mathrm{K}^{+}$-current is induced at positive $V \mathrm{p}$ down to $-30 \mathrm{mV}$ [121]. The opening of $\mathrm{K}_{\mathrm{V}} 2.1$ channels repolarizes $V \mathrm{p}$ and thus closes $\mathrm{Ca}_{\mathrm{V}}$ channels. The action potential spikes return to the plateau $V \mathrm{p}$ of -50 to $-40 \mathrm{mV}$, the level of which is also adjusted by two-pore $\mathrm{K}^{+}$-channels TASK-1 and TALK-1 [122,123]. The ablation of $\mathrm{K}_{\mathrm{V}} 2.1$ indeed reduces $\mathrm{Kv}$ currents by $80 \%$ and prolongs the duration of the action potential, so more insulin is secreted. Mice with ablated $\mathrm{K}_{\mathrm{V}} 2.1$ possess a lower fasting glycemia but elevated insulin and reportedly improved GSIS [124]. In contrast, human $\beta$-cells use $K_{\mathrm{Ca}} 1.1$ channels (also termed BK channels) for the repolarization of $V \mathrm{p}$ [92]. Note also that the downregulation of $\mathrm{K}_{\mathrm{V}}$ was observed after islet incubation with high glucose for $24 \mathrm{~h}$ [125]. 


\subsubsection{Ablations of KIR6.2 and SUR1 Support a Central but not Exclusive Role of $\mathrm{K}_{\mathrm{ATP}}$}

PI $\beta$-cells of mice with ablated Kir6.2 (Kcnj11 gene) (KIR6.2KO mice) did not exhibit typical $\mathrm{K}_{\text {ATP }}$ channel activity, but instead a higher resting $V \mathrm{p}$ and higher intracellular free $\left[\mathrm{Ca}^{2+}\right]_{\mathrm{C}}[126,127]$. Glucose or tolbutamide $\left(\mathrm{K}_{\mathrm{ATP}}\right.$ inhibitor) only transiently elevated these resting free $\left[\mathrm{Ca}^{2+}\right]_{\mathrm{c}}$, and insulin secretion (GSIS) accounted for $<10 \%$ of GSIS in wt mice PIs. Nevertheless, KIR6.2KO islets responded to $30 \mathrm{mM} \mathrm{KCl}$ by secreting insulin, i.e., when plasma membrane depolarization was achieved in this artificial way. $\mathrm{KCl}$-induced depolarization is used by some researchers to model the 2nd GSIS phase. Incubations of KIR6.2KO islets with $10 \mathrm{mM}$ glucose modulated $\mathrm{Ca}^{2+}$ oscillations, and any $V p$ depolarization was $\mathrm{K}_{\mathrm{ATP}}$-independent [127]. Moreover, despite this evident lack of $\mathrm{K}_{\mathrm{ATP}}$ function, KIR6.2KO adult mice had a very mild impairment of glucose tolerance, recovering from the neonatal hypoglycemia and hyperinsulinemia [126]. All these results suggested a lack of the 1st phase of GSIS, but only the attenuated 2nd phase. They can be reinterpreted today by considering other channels substituting for the $\mathrm{K}_{\text {ATP }}$ function in membrane depolarization. Note also that in human pancreatic $\beta$-cells, Kir6.2 loss of function mutations lead to severe hypoglycemia and are accompanied by hyperinsulinemia in some forms [128].

Additionally, one can consider the phenomena observed in mice with ablated SUR1 (Abcc8 gene) $[129,130]$ as separately representing the so-called amplifying pathway of GSIS. SUR1 KO mice had an even milder impairment of glucose tolerance, but exhibit greater fasting hypoglycemia than KIR6.2 KO mice. Their $\beta$-cells exhibited a more depolarized $V p$. Moreover, insulin secretion was only potentiated by GLP-1 or GIP in wt PIs, but not in SUR1 KO PIs, whereas cAMP was elevated in both, reportedly in a PKA-independent way [131]. Since $K_{\text {ATP, }}$ and so also SUR1, reside in IGV membranes, SUR1 KO $\beta$-cells exhibited insufficient IGV docking and fusion with the plasma membrane [132].

2.2.4. Structure of $\mathrm{K}_{\mathrm{ATP}}$ and Behavior with Insulin Non-Stimulating vs. Stimulating Glucose

Despite the resolved structure and numerous mutagenesis studies of $\mathrm{K}_{\mathrm{ATP}}$, no specific amino acid residues have been identified as being redox targets yet. The hetero-octameric $\mathrm{K}_{\mathrm{ATP}}$ contains in total four external regulatory sulfonyl urea receptor 1 (SUR1, a product of $A b c c 8$ gene) subunits and four pore-forming subunits of the potassium inward rectifier Kir6.2 (Kcnj11 gene) $[133,134]$. These four Kir6.2 subunits cluster in the middle of a structure with an $18 \mathrm{~nm}$ diameter and $13 \mathrm{~nm}$ height [135]. The cytoplasm-exposed part of Kir6.2 contains an ATP binding site, $2 \mathrm{~nm}$ below the membrane, which has been traditionally implicated in the channel closing, and an overlapping binding site for phosphatidylinositol 4,5-bisphosphate $\left(\mathrm{PIP}_{2}\right)$. The binding of $\mathrm{PIP}_{2}$ stabilizes the open state. ATP binding to one of four ATP binding sites has already been reported to close the channel [136]. Moreover, the palmitoylation of Cys166 of Kir6.2 was found to enhance its sensitivity to $\mathrm{PIP}_{2}$ [137].

Pharmacologically, $\mathrm{K}_{\text {ATP }}$ is set in the open state by diazoxide, despite high ATP being present [138]. In contrast, sulfonylurea derivatives such as glibenclamide close $\mathrm{K}_{\mathrm{ATP}}$, again independently of ATP, while binding to SUR1. Each of the four SUR1 subunits contain MgATP and MgADP binding sites. MgATP is hydrolyzed at nucleotide binding fold 1 (NBF1) to MgADP and then it activates $\mathrm{K}_{\mathrm{ATP}}$ at NBF2, which is reflected by the ATP-sensitive increase in $\mathrm{K}^{+}$conductance and consequent lower excitability, i.e., also lower sensitivity to ATP inhibition [136].

However, there is a discrepancy that is not yet fully resolved, concerning the drastically different sensitivities of $\mathrm{K}_{\mathrm{ATP}}$ to ATP in vitro vs. in vivo. In inside-out patches used in the patch-clamp methodology, when the cytosolic side is exposed to the experimental medium and when so-called run-down is eliminated, as little as 5-15 $\mu \mathrm{M}$ ATP was able to close the channel [139]. There are much higher $(\mathrm{mM})$ ATP concentrations in intact resting $\beta$-cells, albeit most ATP is bound with $\mathrm{Mg}^{2+}$. Despite the interaction of MgADP with SUR1 decreasing the sensitivity of the whole $\mathrm{K}_{\mathrm{ATP}}$, this phenomenon cannot fully account for the above-mentioned discrepancy. Likewise, the requirement to close only the remaining $7 \%$ population of $\mathrm{K}_{\mathrm{ATP}}$ does not encounter the typical S-shape inhibitory curve with an $\mathrm{IC}_{50}$ 
within the $10 \mu \mathrm{M}$ range. Hence, there must either be endogenous $\mathrm{K}_{\mathrm{ATP}}$ openers or the lack of $\mathrm{H}_{2} \mathrm{O}_{2}$ regulation and/or NSCC contribution could explain this phenomenon.

$A$ variety of molecules were reported to be endogenous $K_{\text {ATP }}$ openers. We already mentioned $\mathrm{PIP}_{2}$, which binds directly to KIR6.2 and decreases the ATP sensitivity of the channel. Upon the release of $\mathrm{PIP}_{2}$ from the binding site, the open probability is decreased $[135,140,141]$. Thus, for example, the extracellular activation of P2Y or muscarinic receptors by autocrine ATP (released together with insulin) decreases PIP $_{2}$ via PLC activation.

\subsubsection{Possible Modulation of $K_{\text {ATP }}$ by Kinases and Phosphatases in Pancreatic $\beta$-Cells}

The phosphorylation of $\mathrm{K}_{\mathrm{ATP}}$ was also thought to set the sensitivity of the ensemble of $\mathrm{K}_{\mathrm{ATP}}$, so that transitions between the two distinct mM ATP concentrations, established by low (3-5 mM) vs. high glucose, will lead to the closing of the remaining fraction of the open $\mathrm{K}_{\text {ATP }}$ channels. Specifically, phosphorylation mediated by PKA could play a major role. Thr224 [142] and Ser372 were established as the candidate PKA phosphorylation sites. Their phosphorylation increases the open probability of $\mathrm{K}_{\mathrm{ATP}}$ in insulin-secreting MIN6 cells [143]. This might hypothetically provide a closing mechanism that acts at higher ATP concentration or even requires $\mathrm{H}_{2} \mathrm{O}_{2}$.

The phosphorylation of $\mathrm{K}_{\mathrm{ATP}}$ also increases the number of channels in the plasma membrane. Thr224 was also found to be phosphorylated by $\mathrm{Ca}^{2+} /$ calmodulin-dependent kinase II (CaMKII) while interacting with $\beta_{\mathrm{IV}}$-spectrin [144]. In vivo, most likely autonomic innervations (maybe also paracrine stimulation) might provide sufficient PKA-mediated phosphorylation of $\mathrm{K}_{\text {ATP }}$. Hence, one should resolve how $\mathrm{K}_{\text {ATP }}$ function relates to phosphorylation in combination with the instantaneous modifications of sulfhydryl groups, which might substantiate the targets of the redox signaling. Thus, sulfhydryl groups of KIR6.2 and/or SUR2 might be affected either by the direct $\mathrm{H}_{2} \mathrm{O}_{2}$ diffusion or by peroxiredoxins and the redox relay system based on their action.

\subsection{Possible Redox Regulation of Ion Channels}

\subsubsection{Observed Redox Regulation of Ion Channels Participating in Insulin Secretion}

Since the pioneering work of Ashcroft and colleagues [145], discovering the essential role of $\mathrm{K}_{\text {ATP }}$ in GSIS, only an indirect inhibition of $\mathrm{K}_{\text {ATP }}$ by $\mathrm{H}_{2} \mathrm{O}_{2}$ was observed in smooth muscle cells [146]. Otherwise, several candidates for redox-sensitive targets have been considered for pancreatic $\beta$-cells: (i) insulin granule exocytosis that might be directly induced by $\mathrm{H}_{2} \mathrm{O}_{2}$, independently of $\mathrm{Ca}_{V}$ triggering [97]. However, we excluded this possibility as being a major contributing factor, since in our experiments with INS-1E cells, independently of glucose, the ability of exogenous $\mathrm{H}_{2} \mathrm{O}_{2}$ to induce insulin secretion was only partially blocked by NOX4-siRNA, but it was fully blocked by the Ca $\mathrm{a}_{\mathrm{L}}$ blocker nimodipine [4]. As a result, despite the used $\mathrm{H}_{2} \mathrm{O}_{2}$ doses exceeding $100 \mu \mathrm{M}$, they did not directly stimulate the $\mathrm{K}_{\mathrm{ATP}}$-independent exocytosis of insulin granules. (ii) $\mathrm{CaV}$ channels, when being coactivated by $\mathrm{H}_{2} \mathrm{O}_{2}$, might hypothetically serve as redox targets or (iii) $\mathrm{H}_{2} \mathrm{O}_{2}$ could hypothetically cause a direct or indirect inhibition of repolarizing $\mathrm{K}^{+}$-channels, such as $\mathrm{K}_{\mathrm{V}}$ [147-149]. Finally, (iv) there could be a competition for NADPH between NOX4 and a hypothetical NADPH-activated $\mathrm{K}^{+}$-channel.

Nevertheless, using patch-clamped INS-1E cells in the cell-attached mode, we demonstrated a closure of $\mathrm{K}_{\text {ATP }}$ by $\mathrm{H}_{2} \mathrm{O}_{2}$ produced by NOX4 at high glucose, since in cells silenced for NOX4, even ATP resulting from the metabolism of high glucose was not able to close the channel [4]. According to our first hypothesis, any of the two $\mathrm{K}_{\text {ATP }}$ components, KIR6.2 or SUR1, should be redox-regulated so that $\mathrm{H}_{2} \mathrm{O}_{2}$ cooperatively with ATP closes the $\mathrm{K}_{\text {ATP }}$ channel (Figure 1A). The redox activation of TPRM2 depolarizing channels, which was already reported $[150,151]$, could be an additional factor. An alternative hypothesis does not require the interaction of $\mathrm{H}_{2} \mathrm{O}_{2}$ with KIR6.2 or SUR1, but expects the essential activation of TRPM2. But this is not supported by the patch-clamp results [4].

Previously, the $\mathrm{Ca}^{2+}$-induced $[97,152]$ or $\mathrm{H}_{2} \mathrm{O}_{2}$-induced exocytosis of insulin granules was reported upon the $\mathrm{H}_{2} \mathrm{O}_{2}$-activation of TRPM2 depolarizing channels [150,151,153]. Hy- 
pothetically, there may be an $\mathrm{H}_{2} \mathrm{O}_{2}$-activated TRPM2-dependent mechanism that provides the essential shift in depolarization to reach the threshold depolarization for $\mathrm{Ca}_{\mathrm{V}}$ in synergy with the $100 \% \mathrm{~K}_{\text {ATP }}$ closure (Figure 1B). This mechanism of shifting depolarization was undoubtedly recognized for the GLP-1 potentiation of insulin secretion via the stimulation of TRPM2 by the GLP1R pathway [154]. Interestingly, it is not Cys residues but Met191 that is responsible for oxidation by $\mathrm{H}_{2} \mathrm{O}_{2}$ in the TRPM2 structure [5], and TRPM2 was also reported to possibly interact with peroxiredoxin $2[114,115]$.

\subsubsection{Possible Redox-Target Residues of KIR6.2, SUR1 and TRPM2}

In our model of Figure 4, we theoretically predicted $\mathrm{H}_{2} \mathrm{O}_{2}$-interacting cysteine residues of known structures of mouse KIR6.2 and hamster SUR1 (checking homologies in mouse vs. hamster sequences) and human TRPM2. We only emphasized those exposed to the cytosolic face of these channels. We based our search on recent findings in the field of redox proteomics by Chouchani's group, who revealed interesting features of the potential ability of Cys to be oxidized [155]. Based on their analysis of 10 different mouse tissues, they proposed that redox-regulated cysteines seem to be tissue specific and that they exist in a local environment of surrounding amino acids, and that this environment affects the Cys reactivity towards redox modifications. At physiological $\mathrm{pH}$, there is equilibrium between the thiolate/thiol group in Cys residues, and this equilibrium is sensitive to electrostatic modulation by nearby amino acids. The analysis revealed that the negatively charged amino acids aspartic and glutamic acid would favor the protonated Cys thiol. In contrast, the positive charge of arginine would stabilize the negatively charged thiolates, which are known to be much more susceptible to redox modifications $[155,156]$.

Based on the above assumptions, we performed an analysis of potentially redoxregulated Cys within structures of mouse/hamster $\mathrm{K}_{\mathrm{ATP}}$ and human TRPM2 channels. Hypothetically, the candidate target cysteines based on this analysis may be susceptible to interactions with $\mathrm{H}_{2} \mathrm{O}_{2}$, and thus may play a role in the above-described redox-activation of GSIS or in insulin secretion stimulated with BCKAs and partly FAs (see below). We searched for a Cys present in the accessible area of the intracellular domains of these proteins with favorable amino acid surroundings. According to the analysis, we found three Cys in the KIR6.2 subunit of the KATP channel (Cys42, Cys197 and Cys 344; Figure 4A), three Cys in the SUR1 subunit of the $\mathrm{K}_{\text {ATP }}$ channel (Cys1378, Cys1487 and Cys1491; Figure 4B) and two Cys in TRPM2 (Cys1250 and Cys1364, Figure 4C). All the suggested Cys share the feature of being in close proximity to an Arg that could increase their reactivity towards redox modification. At this point, it is hard to tell whether such a theoretical prediction has any validity in vivo, and extensive experimental evidence is needed to confirm these suggestions. 


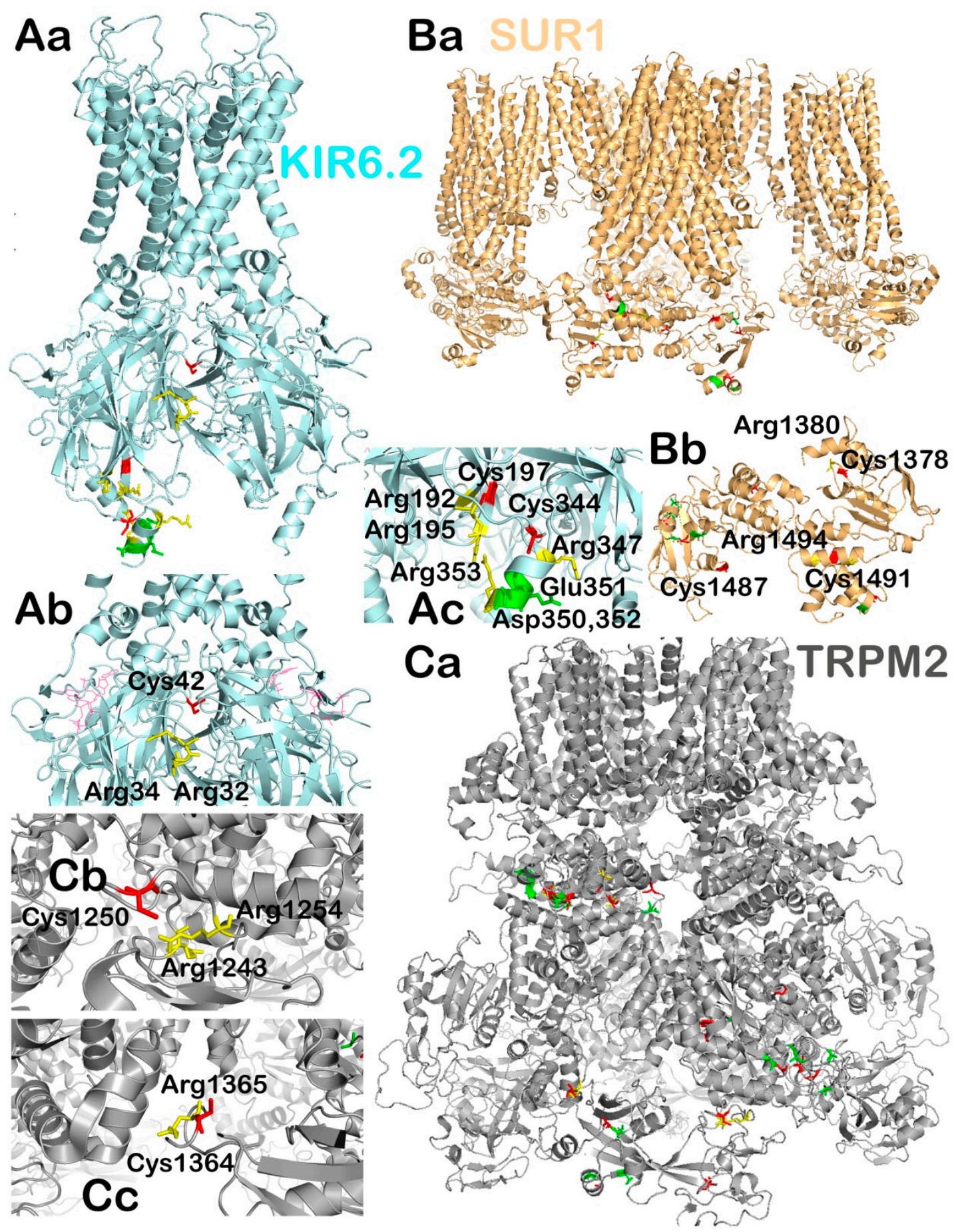

Figure 4. Predicted cysteines interacting with $\mathrm{H}_{2} \mathrm{O}_{2}$ in KIR6.2, SUR1 and TRPM2 structures in pancreatic $\beta$-cells. Synthetic structure of mouse/hamster pancreatic ATP-sensitive potassium channel (pdb code 5wua) [133] and human TRPM2 bound to ADPR and calcium (pdb code 6pus) [157] were used for the visualization of the potential redox reactive cysteines. (Aa,Ab,Ac) Tetrameric assembly of KIR6.2 subunits (light blue) and details of potentially redox regulated Cys with surrounding Arg and/or Asp and Glu, modulating the Cys reactivity towards redox modifications in intracellular regions of the channel; $(\mathbf{B a}, \mathbf{B b})$ prediction of hypothetical redox regulated Cys within one intracellular domain of the SUR1 subunit (light orange) with Arg and Asp/Glu in close proximity and (Ca, $\mathbf{C b}, \mathbf{C c}$ ) human TRPM2 (light grey) and detailed visualization of hypothetical redox regulated Cys in intracellular domains; color coding: Cys in red, Arg in yellow, Asp and Glu in green. 


\subsection{Fine Tuning of the Glucose-Sensitivity Range}

2.4.1. Inhibitory Factor IF1 as a Key Element Setting the Glucose-Sensitivity Range in Pancreatic $\beta$-Cells

Being originally termed an ATPase inhibitory factor 1 (IF1), this factor can now be called ATP-synthase inhibitory factor 1, emphasizing its experimentally evidenced ability to inhibit the synthesis of ATP in vivo $[158,159]$. Previously, it was thought that only the reverse mode of the ATP-synthase is inhibited by IF1, i.e., the mitochondrial ATPase reaction, pumping protons $\left(\mathrm{H}^{+}\right)$to the intracristal space at the expense of ATP hydrolysis to ADP. Such a mode is likely rarely established in primary cells, and the experiments demonstrating the ATPase mode were performed in yeast or cancer cells, in which this mode can coexist with a certain fraction of the ATP synthesis mode.

We recently demonstrated a surprising role of IF1, based on its ability to inhibit ATP synthesis in vivo, thereby adjusting the proper glucose concentration range for GSIS in rat pancreatic $\beta$-cells, INS-1E $[158,159]$. This conclusion was derived from the obtained results, demonstrating that IF1 silencing led to insulin secretion even at very low glucose approaching zero in INS-1E cells [158]. In contrast, IF1 overexpression inhibited GSIS in INS-1E cells [159].

Structurally, dimers of non-phosphorylated IF1 were reported to bridge the $\mathrm{F}_{1}$ moieties of neighboring dimers of the ATP-synthase, covering the crista rims in ordered rows. This was found within a tetrameric structure of the porcine ATP-synthase [160], and we can reasonably expect this to also occur in vivo. However, there is an important distinction in vivo. At most a very small minority of the dimers along the ATP-synthase row of dimers, which form cristae rims could be connected by these IF1-IF1 bridges.

If this was not the case, no ATP synthesis could take place, since IF1 bridging is inhibitory. In reality, IF1 binds at the interface between subunits $\beta$ and $\alpha$ of the $F_{1}$ moiety in proximity to subunit $\gamma$ [161]. Hence, we could hypothetically expect a stoichiometry much lower than the saturating one, which is 1:3 for the IF1: subunit- $\beta$ ratio. Moreover, IF1 was deactivated by mitochondrial PKA-mediated phosphorylation (see Section 2.5.4). Hypothetically, mitochondrial PKA might be involved in some mechanisms of amplification of insulin secretion. Cytosolic PKA, implicated in such amplification, could only participate if IF1 was phosphorylated before its import into the mitochondrial matrix. However, this speculation was not experimentally supported, as well as the speculation that IF1 expression might be even regulated in the translation stage [162]. In turn, experiments suggested a fast IF1 degradation by the factor IEX1 [163]. Consequently, we could expect a multifaceted fine-tuning of IF1 protein content in the matrix, including its phosphorylated fraction.

It is well established that the disruption/inhibition of the ATP-synthase activity induces a higher IMM electrical potential $\Delta \Psi_{\mathrm{m}}$, slowing down the flow of electrons through the RC and thus allowing electron leakage to oxygen with concomitantly enhanced superoxide generation within complexes I and III [16]. Indeed, the upregulation of IF1 protein levels has been linked to increased superoxide formation and the activation of redox-sensitive transcription factors, such as NFKB or HIF1 $\alpha$, causing enhanced proliferation and survival in several cell types and tumors [164]. Consistently, in an INS-1E cell line overexpressing IF1, we observed a significantly higher endurance to stress induced by starvation or various inhibitors of the respiratory chain (Dlasková A., Kahancová A., unpublished). Moreover, it was suggested that IF1 prevents cytochrome $\mathrm{c}$ release during apoptosis via the stabilization of cristae structure due to enhanced ATP-synthase oligomerization [164]. In summary, it is likely that IF1 not only regulates the extent of insulin release, but it might also be critically involved in the regulation of $\beta$-cell number and survival under stressful conditions.

\subsubsection{Cristae Narrowing vs. Steepness of Glucose-Sensitivity Range in Pancreatic $\beta$-Cells}

Due to the unsaturated and perhaps random IF1 bridging of the ATP-synthase dimers along their row forming the crista rim, ATP synthesis and GSIS may also depend on cristae morphology. We found a narrowing of mitochondrial cristae upon GSIS in INS-1E cells [165]. Proton pumps of RC supercomplexes, containing complexes I, III and IV, use the 
energy of electron transfer to pump $\mathrm{H}^{+}$from the matrix space to the intracristal space. Crista junctions (outlets of the intracristal space) prevent the pumped $\mathrm{H}^{+}$from diffusing out to the cytosol via the outer intermembrane space (space between the two cylindrical membranes of mitochondrial tubules, the outer membrane and inner boundary membrane). A local $\mathrm{H}^{+}$coupling via the established protonmotive force $\Delta \mathrm{p}\left(\Delta \mathrm{p}=\Delta \Psi_{\mathrm{m}}+\Delta \mathrm{pH}\right)$ thus occurs within the intracristal space [14]. As a result, any changes in the volume of the intracristal space will affect the coupling efficiency, i.e., the relationship between the $\Delta p$ and the $\mathrm{H}^{+}$backflow through the ATP-synthase $\mathrm{F}_{\mathrm{O}}$ sector back to the matrix.

It remains to be determined whether the observed cristae narrowing upon GSIS also diminishes the volume of the intracristal space. In the positive case, one can predict that crista narrowing in the mitochondria of pancreatic $\beta$-cells strengthens the proton coupling. This would contribute to the steepness of the dose-response relationship of glucose concentration vs. insulin release. Other complex relationships may exist between the IF1 stoichiometry relative to the ATP-synthase, the cristae morphology, and other cristae-shaping proteins such as OPA1 and Mic10.

\subsubsection{Responses of the Mitochondrial Matrix Calcium during GSIS}

Mitochondrial $\mathrm{Ca}^{2+}$ was also recognized to be a significant regulator [166] or participant in the 2nd GSIS phase and in the potentiation of GSIS by GLP-1 [49]. The pioneering work of Denton and colleagues demonstrated the existence of the activation of mitochondrial dehydrogenases by the matrix intake of $\mathrm{Ca}^{2+}$ [167], which, despite the contradictory findings of some researchers [168], could take place upon GSIS [99,169-171] or upon GSIS amplification by GLP-1 $[172,173]$. The mitochondrial matrix concentration $\left[\mathrm{Ca}^{2+}\right]_{\mathrm{m}}$ is regulated by the $\mathrm{Ca}^{2+}$ influx, mediated by the mitochondrial calcium uniporter (MCU) [174], which is balanced by the $\mathrm{Ca}^{2+}$ efflux. The latter is provided by the mitochondrial $\mathrm{Ca}^{2+} / \mathrm{Na}^{+}$ antiporter (NCLX) [175], the fluxes of which are driven by the mitochondrial $\mathrm{Na}^{+} / \mathrm{H}^{+}$antiporter (NHE6), at the expense of the protonmotive force. In some cell types, the existence of the mitochondrial $\mathrm{Ca}^{2+} / \mathrm{H}^{+}$antiporter was also reported. Its expression in $\beta$-cells is ascribed to the LETM1 [176].

When MCU-mediated $\mathrm{Ca}^{2+}$ influx exceeds the $\mathrm{Ca}^{2+}$ efflux, the cytosolic transient $\mathrm{Ca}^{2+}$ elevations caused by the $\mathrm{Ca}_{\mathrm{V}}$ channels are relayed into the mitochondrial matrix [177]. A sudden intake of glucose in primary $\beta$-cells induces the primary $\mathrm{Ca}_{V}$-dependent $\left[\mathrm{Ca}^{2+}\right]_{c}$ oscillations. Those are relayed to somewhat delayed steady-state increases in mitochondrial $\left[\mathrm{Ca}^{2+}\right]_{\mathrm{m}}$, which reach saturation, i.e., maximum values after a certain time $[99,178]$. The $\left[\mathrm{Ca}^{2+}\right]_{\mathrm{m}}$ oscillations, responding to those ongoing in the cytosol, are then superimposed onto the linearly increasing $\left[\mathrm{Ca}^{2+}\right]_{\mathrm{m}}$ up to saturation. When bursting occurred at $10 \mathrm{mM}$ glucose, the higher frequency of action potential spikes within a burst led to a higher amplitude of the $\left[\mathrm{Ca}^{2+}\right]_{\mathrm{m}}$ increase, i.e., higher saturating values [178]. These changes induced a biphasic increase in the ATP/ADP ratio, with its 2nd part after 5 min $[99,177,178]$.

MCU-deficient $\beta$-cells did not exhibit the 2nd part of the increase in ATP/ADP [99]. Note that this does not necessarily correspond to the 2nd GSIS phase. When the artificial overexpression of the $\mathrm{Ca}^{2+}$ binding protein S100G in the mitochondrial matrix of INS-1E cells allowed declines in the matrix $\mathrm{Ca}^{2+}$ independently of the cytosolic $\mathrm{Ca}^{2+}$, the glucosestimulated $\mathrm{NAD}(\mathrm{P}) \mathrm{H}$ formation was prevented, and increases in mitochondrial respiration and OXPHOS [179]. Thus, glucose-induced $\left[\mathrm{Ca}^{2+}\right]_{\mathrm{m}}$ elevations reached up to $880 \mathrm{nM}$ in wt cells, whereas only $530 \mathrm{nM}$ upon the S100G overexpression, which attenuated GSIS. In contrast, the overexpression of S100G in islet $\beta$-cells did not decrease the 1st phase of GSIS, but attenuated the 2nd phase.

\subsection{Amplifying Mechanisms}

\subsubsection{Receptor Mediated Amplification}

$G$ protein-coupled receptors provide a wide range of cell responses that are mutually interrelated. They activate heterotrimeric $G$ proteins, which typically regulate the production of second messengers or signals via other proteins. Thus, the $G$ protein $G \alpha s$ 
increases the generation of cyclic AMP (cAMP), whereas Gai/o decreases it [180-182]. G $\alpha \mathrm{q} / 11$ initiates the PLC-mediated hydrolysis of phosphatidylinositol 4,5-bisphosphate $\left(\mathrm{PIP}_{2}\right)$ into diacylglycerol (DAG) and IP3 $[183,184]$. G $\alpha 12 / 13$ promotes the protein RhoA for remodeling the cytoskeleton [185]. The class of proteins termed $\beta$-arrestins initiate signaling via the proximal MAP kinase, IKB and Akt pathways [186]. Downstream pathways are discussed below, which predominantly lead to either (i) modulation of the plasma membrane channels, typically $\mathrm{Ca}_{\mathrm{V}}, \mathrm{K}_{\mathrm{ATP}}$ and $\mathrm{K}_{\mathrm{V}}$, to provide more intensive insulin secretion; (ii) stimulation of the surplus $\mathrm{Ca}^{2+}$ influx to the cytosol from ER or other stores and (iii) influencing the kinetics of the IGV in its docking, priming and fusion with the plasma membrane. Note, that only (iii) mechanisms could be independent of $\mathrm{Ca}_{\mathrm{V}}$, if other factors provided sufficient $\left[\mathrm{Ca}^{2+}\right] \mathrm{c}$ increase. Most likely, however, there is usually a synergy between all of these mechanisms (i) to (iii). The general role of second messengers in pancreatic $\beta$-cells is described below, while the participation of each pathway in insulin secretion stimulated by various secretagogues is described in later sections.

\subsubsection{PKA Pathway in Pancreatic $\beta$-cells}

The activation of $G \alpha$ s increases the activity of plasma membrane transmembrane adenylate cyclases (tmAC) producing cAMP from ATP [181,182]. A number of phosphodiesterases of 11 families then degrade cAMP (some also or exclusively cGMP). cAMP is a universal 2nd messenger providing a vast number of diverse physiological functions, being involved in metabolism, differentiation, synaptic transmission, ion channel activity, growth and development or in cellular level in cell migration, differentiation, proliferation and apoptosis. There are also soluble adenylate cyclases (sACs), whose reaction is potentiated by $\mathrm{Ca}^{2+}$ and bicarbonate. One such sAC is also located in the mitochondrial matrix of pancreatic $\beta$-cells [187]. The major mediator of cAMP effects is the cAMP-dependent PKA [188]. Note that PKA is ubiquitous in every mammalian cell and acts in the cell cytosol, nucleus and evidence has also been gathered that it acts in mitochondria, either tethered to the outer mitochondrial membrane (OMM) or located in the matrix $[189,190]$.

PKA is a heterotetramer of two distinct catalytic subunits and a dimer of a regulatory subunit. Three distinct catalytic $(\mathrm{C} \alpha, \mathrm{C} \beta$, and $\mathrm{C} \gamma)$ and four isoforms of the regulatory subunit exists (RI $\alpha, R I \beta, R I I \alpha$ and RII $\beta)$, which bind cAMP [188]. After cAMP binding, the catalytic subunits dissociate and phosphorylate a vast number of proteins. PKA is tethered by A-kinase anchoring proteins (AKAPs), localizing it to specific sites in the cell [189], e.g., externally to the OMM from the cytosolic side. The PKA is thus positioned in close proximity to the dedicated protein substrates. Phosphodiesterases (PDEs) degrade cAMP and contribute to the termination of signaling. For example, in $\beta$-cells PDE3B attenuates the potentiation of insulin secretion by GLP-1 [191].

In pancreatic $\beta$-cells, the PKA pathway is involved in the signaling of incretin (typically GLP-1 and GIP) receptors [180], and makes a minor contribution to signaling from metabotropic receptors, such as GPR40, sensing fatty acids [184]. PKA typically amplifies the $\mathrm{Ca}^{2+}$-dependent exocytosis of insulin granules. The core pathway involves the PKA-mediated phosphorylation and hence activation of the $\mathrm{Ca}_{V} \beta 2$-subunit, in concert with the phosphorylation of $\mathrm{K}_{\mathrm{ATP}}$, increasing the ATP concentration range required for its closure [192]. In addition, PKA inhibits Kv channels, which otherwise terminate plasma membrane depolarization, hence this prolongs the already more intensive $\mathrm{Ca}^{2+}$ influx via phosphorylated $\mathrm{Ca}_{V}$ channels and hence prolongs/intensifies the exocytosis of IGVs [193].

An exocytosis modulating the protein snapin is another important target of PKA. The phosphorylation of snapin enables its interaction with the other proteins of the IGV, thus potentiating the 1st GSIS phase [194]. Snapin participates in tethering IGVs to the plasma membrane by coiled-coil interaction with a lipid-anchored protein, the synaptosomal nerve-associated protein 25 (SNAP-25) [195]. 


\subsubsection{EPAC2 Pathway in Pancreatic $\beta$-Cells}

The PKA pathway provides approximately $50 \%$ of the responses to cAMP in $\beta$ cells [196]. The parallel pathway of enhanced signaling via exchange proteins directly activated by cAMP 2 (EPAC2) enables the remaining portion [197-199]. Due to its guanine nucleotide exchange activity, the EPAC2 protein provides $\mathrm{Ca}^{2+}$-induced $\mathrm{Ca}^{2+}$ release from the ER via RyR [200], although the abundance of RyR in $\beta$-cells has been questioned [201]. Nevertheless, the RNAseq approach indicated the existence of RyR transcript in mouse and human $\beta$-cells. The $\mathrm{Ca}^{2+}$ release from ER via RyR can only happen upon abundant glucose intake, leading to the primary $\mathrm{Ca}_{V}$ opening [202]. The primary increase in $\left[\mathrm{Ca}^{2+}\right]_{\mathrm{c}}$ then reaches a threshold, at or above which the RyR $\mathrm{Ca}^{2+}$ channel would be open. Note also that sustained $\mathrm{Ca}_{V}$ opening partially refills the $\mathrm{ER} \mathrm{Ca}^{2+}$ stores, hence counteracting the above effect. The most important effect of EPAC2A is the direct activation of TRPM2, essential for $\mathrm{K}_{\mathrm{ATP}}$-triggered GSIS, i.e., providing an NSCC-mediated depolarization shift of up to $-50 \mathrm{mV}$ [154]. The redox status of TRPM2 under these conditions is not known.

The EPAC pathway also affects IGV proteins and therefore facilitates IGV exocytosis. Indeed, the cAMP potentiation of insulin secretion via distinct EPAC2 pathways involves the regulation of several IGV-associated proteins. The role of Rim $2 \alpha$ GTPase was elucidated $[203,204]$. Rim $2 \alpha$ is located on the inner plasma membrane surface and on the membranes of insulin granules. Thus, $\operatorname{Rim} 2 \alpha$ represents a scaffold that enables the regulated exocytosis of insulin granules [205]. While interacting with another GTPase Rab3A, located on insulin granules, the Rim $2 \alpha-\operatorname{Rab} 3 \mathrm{~A}$ complex enables the docking of IGVs (Figure 2). Priming is then initiated when Rim $2 \alpha$ interacts with mammalian uncoordinated homology 13-1 (Munc13-1), which subsequently opens syntaxin 1 to interact with the IGVs. This is followed by fusion with the plasma membrane. Rim $2 \alpha$ double mutants K136E/K138E locked Rab3A to Rim $2 \alpha$ so that priming cannot proceed and syntaxin 1 remains closed.

Interestingly, EPAC2 also binds to the nucleotide binding domain-1 of the SUR1 $\mathrm{K}_{\mathrm{ATP}}$ subunit. The EPAC2 release from SUR1 is then provided by cAMP [25]. Hypothetically, this locally released EPAC2 induces the release of $\operatorname{Rim} 2 \alpha$ from the $\alpha 1.2 \mathrm{Ca}_{\mathrm{L}}$ subunit [180]. Ongoing $\mathrm{Ca}^{2+}$ influx facilitates EPAC2 binding to Rim $2 \alpha$ and trimers of these two proteins with another $\mathrm{Ca}^{2+}$ sensor, Piccolo. The trimers interact with Rab3A and thus enable IGV exocytosis.

\subsubsection{Mitochondrial PKA Pathways in Pancreatic $\beta$-cells}

It has been found that PKA phosphorylates numerous mitochondrial proteins, including proteins of the mitochondrial matrix [206,207]. Therefore, we must consider PKA to be a parallel effector that also involves cAMP signaling in the mitochondrial matrix of pancreatic $\beta$-cells $[189,190]$. Suitable protein residues exposed to the cytosolic face of the OMM are phosphorylated by the PKA residing on the OMM (PKA $\mathrm{PMM})$. The PKA attachment is mediated by the OMM-anchored AKAP. In this way, the fission/fusion dynamics of the mitochondrial network are regulated, besides the protection of apoptosis. As a result of this OMM PKA signaling, typically mitochondrial tubule elongation is promoted within the mitochondrial network in the cell, with concomitantly increased mitochondrial membrane potential, besides numerous other phenomena. Additionally, the complex IV COXIV-1 subunit (which prevents its inhibition by ATP) is phosphorylated by PKA from the intracristal or the outer intermembrane space $\left(\mathrm{IMS}_{\text {out }}\right)$, i.e., the space between the OMM and the inner boundary membrane (IBM) [208]. The IBM is an unfolded IMM part forming a cylindrical IBM-IMS out $_{-} \mathrm{OMM}$ sandwich.

From the unambiguous fact that only the non-phosphorylated IF1 inhibits the ATPsynthase, one can derive a hypothesis that expects mtPKA localized in the mitochondrial matrix to activate the ATP-synthase by providing phosphorylation of the major ensemble of IF1 protein. This was already verified in INS-1E cells [158,159]. The question is whether such regulation can be acute, i.e., whether it is in effect during the 1st or 2nd GSIS phase. Evidence has accumulated for the existence of the PKA pathway in the mitochondrial 
matrix. All the necessary components were identified to be localized in the matrix, including sAC, PDE2A2 [209] and also the matrix PKA (mtPKA) [210], albeit its existence is still controversial. A pharmacological activator of GPRs, forskolin, was reported to induce the phosphorylation of matrix proteins. The ATPase inhibitory factor IF1 was reported to be phosphorylated. However, there is still disagreement over whether this takes place in the mitochondrial matrix [164] or whether IF1 is phosphorylated by the cytosolic or OMM-residing PKA prior to its import to the matrix [211]. There was also a consensus that cAMP cannot freely diffuse to the matrix [209]. Thus, cAMP located in the mitochondrial matrix represents an independent pool, which may therefore provide an independent regulation $[212,213]$.

The most likely source of matrix cAMP is the matrix sAC activated by bicarbonate [187] and $\mathrm{Ca}^{2+}$, though it was reported that a matrix cAMP increase does not activate mtPKA [214]. Since $\mathrm{CO}_{2}$ is increasingly released when the Krebs cycle turnover increases upon the metabolic stimulation of insulin secretion, we can hypothesize that the elevation of cAMP is independent of the cytosolic cAMP signaling in pancreatic $\beta$-cells. Additionally, the basic $\left[\mathrm{Ca}^{2+}\right]_{\mathrm{C}}$ increase due to $\mathrm{Ca}_{\mathrm{V}}$ opening and its relaying to a $\left[\mathrm{Ca}^{2+}\right]_{\mathrm{m}}$ increase should activate sAC. The main effector is most likely the matrix-localized mtPKA [215], although nothing is yet known about how PKA is translocated into the mitochondrial matrix. The matrix localization of PKA was clearly demonstrated in Drosophila [210].

In any case, OXPHOS is facilitated in the mitochondria of numerous tissues, stemming from the phosphorylation of complex I NDUFS4 subunit (facilitating its Hsp70mediated import) and IF1 (enhancing ATP synthesis by disabling the inhibitory binding of phosphorylated IF1 dimers to the ATP synthase; see above) [211] and there is no reason for this to not also be the case in pancreatic $\beta$-cells. Indeed, this was confirmed $[158,159]$. The observed release of the PKA catalytic subunits by the increased ROS is also noteworthy $[216,217]$.

We may hypothesize that the metabolic branch of the amplification of insulin secretion originates from the elevated mitochondrial matrix cAMP and activated mtPKA, at least due to the increased $\mathrm{CO}_{2}$ output in the Krebs cycle and increased $\mathrm{Ca}^{2+}$ uptake into the mitochondrial matrix. Note that $\mathrm{CO}_{2}$ is converted to bicarbonate by the carbonic anhydrase [218]. Thus mtPKA could accompany the cytosolic PKA signaling initiated by the GPR40 and GLPR or GIPR receptors. In other words, mtPKA signaling should contribute to the amplification of insulin secretion by FAs or incretins while phosphorylating at least IF1, and probably also complex I.

We should also note that a partial suppression of ATP synthesis is also projected into the RC-produced superoxide. Indeed, RC proton pumps work faster at faster ATPsynthesis, given by the $\mathrm{H}^{+}$backflow to the matrix. When the latter is retarded, such as due to the binding of non-phosphorylated IF1 dimers, then RC proton pumps also retard their $\mathrm{H}^{+}$flux against $\Delta \mathrm{p}$, while RC electron transfer also slows down. This leads to a higher superoxide formation, which would otherwise decrease upon the increasing ATP-synthesis. In this way, the mtPKA-IF1 pathway may slightly contribute to the redox signaling upon GSIS, adding to the $\mathrm{H}_{2} \mathrm{O}_{2}$ fraction resulting from surplus superoxide that is formed and converted upon slightly retarded ATP synthesis.

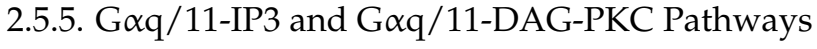

The $G$ protein $\mathrm{G} \alpha \mathrm{q} / 11$ initiates signaling through the phospholipase $\mathrm{C}$ (PLC)-mediated hydrolysis of phosphatidylinositol 4,5-bisphosphate into DAG and IP3 [183]. The most important contribution of DAG is the facilitation of the threshold plasma membrane depolarization, via the protein kinases C- (PKC-) mediated phosphorylation of TRPM4 and TRPM5, leading to their activation. These channels belong to the essential synergic channels for $\mathrm{K}_{\mathrm{ATP}}$ [219]. PKCs hence probably contribute to the required essential $V \mathrm{p}$ shift, otherwise provided by TRPM2. One of the effectors of IP3 is the IP3 receptor (IP3R; subtypes IP3R1, IP3R2 and IP3R3), forming another important $\mathrm{Ca}^{2+}$ channel residing on the ER membrane in $\beta$-cells [220]. Similarly to the EPAC2-RyR route of $\mathrm{Ca}^{2+}$ release from the 
ER, the opening of this channel amplifies the primary $\mathrm{Ca}_{\mathrm{V}}$-mediated $\mathrm{Ca}^{2+}$ signaling for insulin release.

PKCs isoenzymes, which belong to the family of serine/threonine kinases, are activated by DAG, which is their main effector [221-224]. PKCs are important for a broad range of cellular processes [225]. Pancreatic $\beta$-cells express members of both conventional PKCs (cPKCs), which are activated by DAG and $\mathrm{Ca}^{2+}$, and "novel" PKCs (nPKCs), which are activated by DAG, but not by $\mathrm{Ca}^{2+}$. This enables nPKCs to be activated by a general PLC-mediated hydrolysis of lipids other than PIP 2 . The activation of cPKCs and nPKCs typically involves their translocation to the plasma membrane [223]. DAG-stimulated nPKCs reportedly also migrate to the Golgi apparatus and mitochondria (PKC $\delta$ to nucleus) [225]. PKC colocalization with the OMM has been reported [226]. The translocation dynamics of various fluorescence-tagged PKC isoforms together with DAG dynamics, and the subplasma membrane $\left[\mathrm{Ca}^{2+}\right]$, were analyzed in insulin-secreting $\beta$-cells using TIRF [223]. The results revealed that insulin secretagogues induce transient DAG microdomains, which rapidly recruit both cPKCs (specifically isoforms PKC $\alpha, P K C \beta I$ and PKC $\beta I I$ ) and nPKCs (isoforms PKC $\delta, P K C \varepsilon$ and PKC $)$ ), emphasizing the role of DAG in the rapid kinetic control of PKC-mediated phosphorylation. The pharmacological stimulation of GPR40 also activated PKC $\varepsilon$ at a substimulatory (glucose), while in addition to PKC $\varepsilon$, PKC $\alpha$ was also activated at high insulin-stimulating (glucose) [224]. nPKCs were found to affect cytoskeleton dynamics in a way that eases IGV exocytosis [227]. Besides their canonical plasma membrane effects, PKC [227] and downstream ERK1/2 signaling stimulate OXPHOS, and hence mitochondrial ATP synthesis [227]. Thus, PKC signaling was claimed to target mitochondria $[225,228]$, since a range of nPKC inhibitors prevented phorbolester-induced respiration in INS-1E cells and human PIs [227]. However, the elevated OXPHOS was affected indirectly via c-Raf-MEK-ERK1/2 signaling. Thus, the existence of matrix-located PKC in pancreatic $\beta$-cells is not clear.

\subsection{Biology of Insulin Granule Vesicles}

\subsubsection{Biogenesis of Insulin Granule Vesicles}

About 10,000 granules are present in rodent $\beta$-cells [229]. Besides insulin hexamers crystallizing with zinc ions $\mathrm{Zn}^{2+}$, IGVs contain C-peptide, ATP, glutamate, GABA, ghrelin and islet amyloid polypeptide (IAPP, termed also amylin). All these factors exhibit an autocrine function. IGVs have their own membrane, ion channels and protein machinery, which is not completely understood [75]. Thus, IGVs represent self-contained organelles that are $200-400 \mathrm{~nm}$ in diameter, apparent in electron micrographs as having a dense core with a white halo in rodents $[77,230]$. IGVs contain a high content of total $\mathrm{Ca}^{2+}$ (that would correspond to $120 \mathrm{mM}$ ), most of which is bound [231]. The free $\mathrm{Ca}^{2+}$ concentration may correspond to $40 \mu \mathrm{M}$ [232].

The biogenesis of IGVs is initiated by the signal of high glucose, which serves for the instant maintenance of replenished IGVs $[77,233]$. Thus, the translation of preproinsulin is dependent on glucose [233], which is followed by the processing of proinsulin in the ER [234] and the transition to the Golgi apparatus and its trans Golgi network, from which nascent IGVs bud [235]. In these nascent IGVs, $8.93 \mathrm{kDa}$ proinsulin is cleaved by prohormone convertases (losing a C-peptide), and insulin is matured as a 51-residue $5.808 \mathrm{kDa}$ protein and crystallized into hexamers in association with $\mathrm{Zn}^{2+}[230,236]$ and bound $\mathrm{Ca}^{2+}$ [49]. About 8-9 fg (1.7 attomoles) of insulin gives a $100 \mathrm{mM}$ concentration in a single IGV. All the insulin accounts for $5-10 \%$ of $\beta$-cell proteins [75]. Additionally, microtubules were recently implicated in this process, with the involvement of the EPAC2 pathway increasing the formation of such microtubules derived from the Golgi apparatus [237]. The process was found to support both phases of insulin secretion. The nascent IGVs are transformed into immature IGVs (less strongly stained with a thin halo or without halos) coated with clathrin, while acidification excludes the clathrin-coated membrane parts, which produces the mature IGVs [42]. The transfer of IGVs towards the plasma membrane is achieved through sliding along microtubules. 


\subsubsection{Major Proteins of IGVs}

Proteomic analysis indicated numerous proteins contained in IGVs [238]. Besides insulin, numerous enzymes were found inside these vesicles, such as chromogranin- $\mathrm{A}$, carboxypeptidase E, ectonucleotide pyrophosphatase/phosphodiesterase family member 2, fibronectin, stanniocalcin-1, several neuroendocrine convertases, sulfhydryl oxidase, $2^{\prime}, 3^{\prime}-$ cyclic-nucleotide $3^{\prime}$-phosphodiesterase, carboxypeptidase N, $\beta$-galactosidase and others. Notable proteins found attached to or within the IGV membranes were synaptotagmin-like protein 4, Rab37, Rab1 $\alpha$, vesicle-associated membrane protein 3 (VAMP3), syntaxin-12, syntaxin-5, V-type $\mathrm{H}^{+}$ATPase, receptor-type tyrosine-protein phosphatase N2, SLC12A9, carboxypeptidase $\mathrm{D}$ and transferin receptor protein 1, among numerous others. Several vesicle-associated membrane proteins (VAMP), notably synaptobrevin/VAMP2, reside on the IGV surface [238]. Synaptobrevins and similar IGV proteins are termed vSNARE to distinguish them from the target SNARE proteins of the plasma membrane. As described below, these soluble N-ethylmaleimide-sensitive factor (NSF) attachment protein receptor (SNARE) proteins are key proteins that provide the exocytosis of IGVs by allowing the fusion of two membranes.

IGV membranes contain zinc transporter member 8 (ZnT8/SLC30A8), which allows a high accumulation of $\mathrm{Zn}^{2+}$ in the IGV lumen to promote the insulin crystalline form [239]. During IGV exocytosis, thus also $\mathrm{Zn}^{2+}$ is released together with C-peptide in addition to the crystalline insulin, ATP, GABA, glutamate, ghrelin, IAPP/amylin and other components. Surprisingly, channels in the IGV membrane involve the $\mathrm{K}_{\text {ATP }}$ channels $[240,241]$, ClC3 channels (see below), TRPV5 [242], RyR [243] and probably also IP3R [244]. The latter three channel types serve for $\mathrm{Ca}^{2+}$ mobilization from IGVs, thus contributing to $\mathrm{Ca}^{2+}$ oscillation dynamics. The role of $\mathrm{K}_{\text {ATP }}$ in IGVs is less clear. Some clues could be provided by the interaction of the small cytoplasmic phospholipid binging protein $\alpha$-synuclein with the $\mathrm{K}_{\mathrm{ATP}}$ of IGVs, which inhibits the basal insulin release, but not GSIS [241].

Overall changes in IGV interiors are given by the V-type $\mathrm{H}^{+}$ATPase, which pumps $\mathrm{H}^{+}$into the IGV lumen, hence establishing protonmotive force with the electrical potential $\Delta \Phi_{\mathrm{IGV}}$ and $\Delta \mathrm{pH}_{\mathrm{IGV}}$ components. The concomitant $\mathrm{Cl}^{-}$influx, mediated by the $\mathrm{ClC} 3$ channel, contributes to $\Delta \mathrm{pH}_{\mathrm{IGV}}$. Similarly, $\Delta \Phi_{\mathrm{IGV}}$ and $\Delta \mathrm{pH}_{\mathrm{IGV}}$ drive all transport processes across the IGV membrane. The ATPase $\mathrm{H}^{+}$-transporting lysosomal accessory protein 2 (ATP6ap2) is required for the assembly of $\mathrm{V}-\mathrm{H}^{+}$ATPase in IGVs. Reportedly, ATP6ap2 interacts with GLP1R, while its silencing decreased both GSIS and its potentiation by GLP-1 in INS-1E cells [245].

\subsubsection{Mechanism of IGV Exocytosis}

A small portion of insulin was found to be released without being contained in IGVs [246]. IGV release is pulsatile, in accord with the $\mathrm{Ca}^{2+}$ oscillations, and not continuous [247]. When accounting for averaged rates, a fraction of $0.14 \% \mathrm{~min}^{-1}$ of IGVs was found to be released in the 1 st phase and $0.05 \% \mathrm{~min}^{-1}$ in the 2nd phase of GSIS $[42,68,230]$. The latter would account for $3 \%$ of IGVs after $60 \mathrm{~min}$ from those existing prior to the transition to a high glucose. In a snapshot, up to $1 \%$ of IGVs were characterized as readily released, $7 \%$ IGVs were found to be docked to the plasma membrane, $15 \%$ were found near the plasma membrane, but not docked and the remaining 77\% of IGVs represent a large reserve pool [79]. Docking was found to not be essential, but produced a delay in fusion with the plasma membrane [248].

The major $\mathrm{Ca}^{2+}$ signal preceding insulin secretion allows $\mathrm{Ca}^{2+}$ binding to the $\mathrm{C} 2$ domains of complexin- 1 and C2A and C2B domains of certain synaptogamins $[249,250]$ (Figure 5). So-called priming of IGVs follows and involves a conformation change from the closed to the open form of the triple- $\alpha$-helical Habc N-terminal domain of syntaxin1 (Figure 5, transition from the stage 1 to stage 2). The mammalian uncoordinated-18 protein (Munc18-1) binds to the closed form, preventing any participation in the SNARE complex [251]. Additionally, Munc13-1 facilitates the syntaxin transition in concert with some other proteins [249]. In parallel with synaptogamin activation by $\mathrm{Ca}^{2+}$, the plasma 
membrane proteins syntaxin-1, SNAP-25 and other target-SNARE proteins attract IGVs via synaptobrevins and other VAMP proteins, while forming a coiled-coil quaternary structure [252]. The inherent part of such a complex is given by the $\mathrm{Ca}^{2+}$-bound protein, termed complexin-1 (Figure 5, stage 2). These events build a four-component bundle coiled-coil SNARE complex.

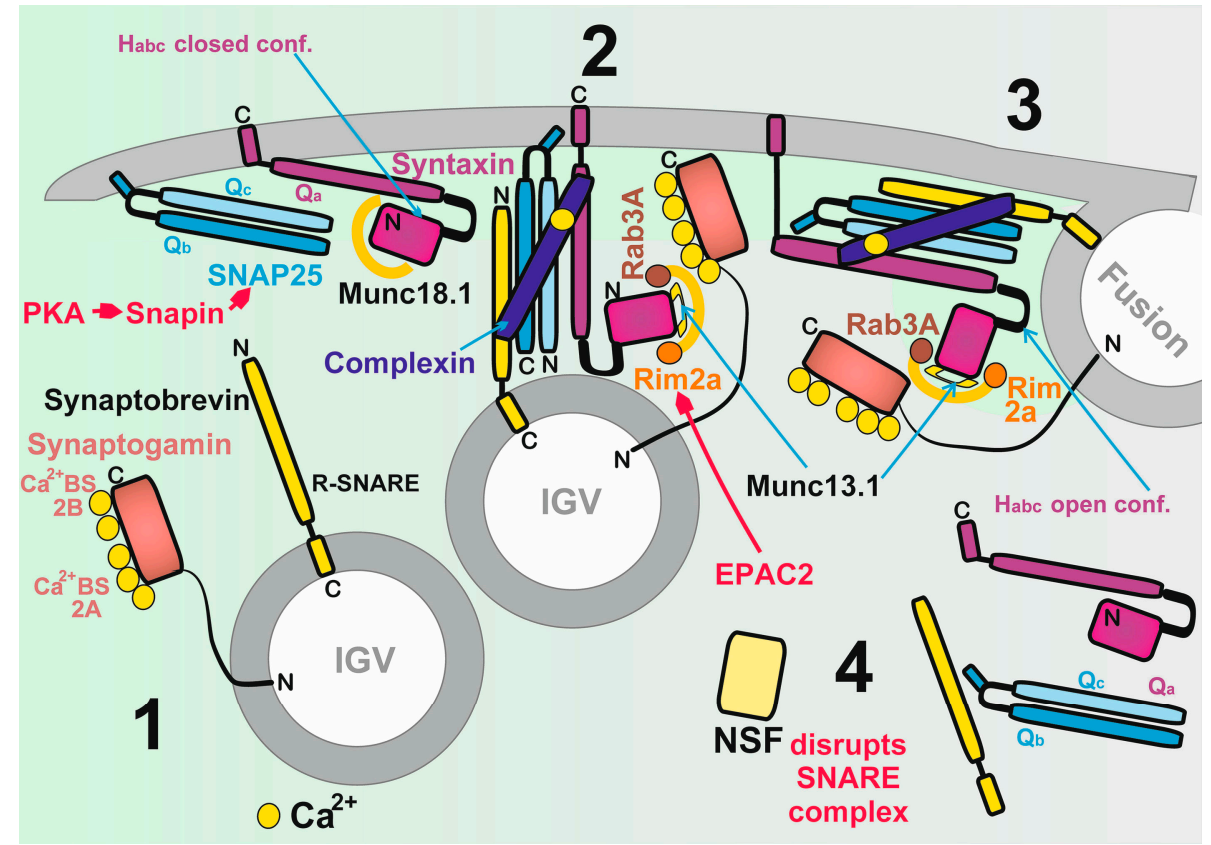

Figure 5. Mechanisms of insulin granule vesicle (IGV) exocytosis, schematically illustrated in four stages: $\mathrm{Ca}^{2+}$ ions are depicted as yellow circles. $\mathrm{C}$ - and $\mathrm{N}$-ends of proteins are marked and specific protein domains. Stage 1, when the Munc18.1 protein binds the Habc domain of syntaxin-1, locking it in the closed conformation. The elevated $\mathrm{Ca}^{2+}$ promotes $\mathrm{Ca}^{2+}$ binding to the synaptogamin $\mathrm{C} 2$ domains at the $\mathrm{Ca}^{2+}$ binding sites $2 \mathrm{~B}$ and $2 \mathrm{~A}$ (" $\mathrm{Ca}^{2+} \mathrm{BS} 2 \mathrm{~B}$ and $\left.2 \mathrm{~A}^{\prime \prime}\right)$ and to the $\mathrm{C} 2$ domain of the complexin. In stage 2, priming by the Rim $2 \alpha$ and Rab3A GTPases and their interaction with the Munc13.1 alters the syntaxin-1 conformation to the open one. This allows the formation of a large SNARE complex, the four-component bundle coiled-coil SNARE complex. In stage 3, SNARE complexes in a zippering fashion have enough energy to move the vesicle into close proximity to the plasma membrane and ensure fusion. At first, a fusion stalk (not shown) is formed followed by a larger septum until the content of the IGV lumen relocates to the outside of the cell. Along the pore expansion, the cis to trans conformation change of the SNARE complex takes place. In the terminating stage 4 , the large SNARE complex is disrupted by the NSF ATPase. The verified PKA and EPAC2 targets are also shown. Note, that certain synaptogamin isoforms such as isoforms 4 and 8 do not bind $\mathrm{Ca}^{2+}$. If these were present in $\beta$-cells, this would allow IGV exocytosis at lower $\mathrm{Ca}^{2+}$ concentrations. However, $\mathrm{Ca}^{2+}$ would still be required for complexin.

The resulting SNARE core complex relocates closer to the plasma membrane so that the IGV membrane is moved into its proximity, and thus facilitates establishment of the so-called fusion stalk. Further zippering of coiled-coil structures allows the fusion of a larger part of the IGV membrane with the plasma membrane, until a fusion pore is formed [250,252]. A further expansion of the fusion pore is promoted due to the cis to trans conformation of the SNARE complex and, simultaneously, the entire IGV lumen content is relocated to the cell exterior (to islet capillaries) (Figure 5, stage 3). Note that also the PKA-phosphorylated Snapin aids the IGV tethering to the plasma membrane by coiled coil interaction with a protein SNAP-25, which is anchored to the plasma membrane by a palmitoyl chain interacting with the cysteines of the random coil linker region joining the $\mathrm{Qb}$ and Qc domains [195]. 
As was mentioned above, small scaffold GTPases Rim $2 \alpha$ and Rab3A, in a complex, interact with Munc13-1, which subsequently opens the syntaxin. Being GTPases, these proteins are activated by the cAMP-dependent EPAC2A pathway. In the end, the NEM sensitive factor (NSF), being a homo-hexameric AAA-type ATPase, disrupts the SNARE complexes at the expense of ATP hydrolysis (Figure 5, stage 4), so the exocytosis is completed. Palmitoylated SNAP-25 dissociates automatically from the membrane upon fusion.

\subsubsection{Glutamate Promotion of Insulin Granule Vesicles Exocytosis}

The originally described key role of glutamate in relation to GSIS [253-255] was questioned by subsequent experiments [256,257]. In turn, total internal reflection fluorescence microscopy (TIRF) studies demonstrated that membrane-permeant dimethyl-glutamate amplified both phases of GSIS with respect to the frequency of IGV merging with the plasma membrane [258]. All previous controversial findings thus probably reflect the specific uptake of glutamate into IGVs driven by $\Delta \mathrm{pH}_{\mathrm{IGV}}$, established by the $\mathrm{V}-\mathrm{H}^{+}$ATPase [259]. Anion influx, namely $\mathrm{Cl}^{-}$influx by $\mathrm{ClC} 3$, helps to build $\Delta \mathrm{pH} \mathrm{H}_{\mathrm{IGV}}$.

The glutamate concentration inside the IGV lumen is maintained by the EAAT2mediated efflux, while being balanced by the uptake into the lumen, mediated by vesicular glutamate transporters VGLUT1, VGLUT2 and VGLUT3. All these reside in the vesicular membranes, being differentially expressed in various cultured model $\beta$-cells and islet $\beta$-cells [260]. Their ablation did not affect GSIS, but reduced the incretin-induced amplification of GSIS [258]. The impaired glutamate vesicular transport thus decreases overall insulin secretion. Glutamate dependence was also judged from experiments with ablation of the glutamine transporter of the plasma membrane, sodium-coupled neutral amino acid transporter 5 (SNAT5). The insufficient glutamine uptake and concomitant glutaminase reaction, converting glutamine to glutamate, reportedly lead to the reduced GLP-1 amplification of GSIS [261].

\section{Incretins: GLP-1 and GIP}

\subsection{Incretin Potentiation of GSIS In Vivo}

Glucagon-like peptide 1 (GLP-1) and gastric inhibitory polypeptide (GIP) have a prominent impact among other peptides belonging to the incretins. Oral glucose administration provides a much higher insulin secretion response compared to parenteral administration [262]. The potentiation surplus can be about equally ascribed to GLP-1 and GIP [263]. This is reflected, for example, by the diminished insulin secretion response to oral glucose in GLP-1 knockout mice [264,265], which decreased even more when double knockout (GLP-1 plus GIP) mice were tested [265].

Incretins (GLP-1 or GIP) massively amplify GSIS by signaling via G $\alpha$ s proteins, $\mathrm{tmAC}$, cAMP and subsequently via both PKA-dependent and EPAC2A-dependent pathways. As described above, the phosphorylation by PKA increases the sensing range of $K_{\text {ATP }}$ to physiological $\mathrm{mM}$ ATP, inhibits $\mathrm{K}_{\mathrm{V}}$, activates $\mathrm{Ca}_{\mathrm{V}}$ [266], the GLUT transporter and snapin [194,195]. Simultaneously, the EPAC2A pathway directly activates TRPM2, essential for $\mathrm{K}_{\mathrm{ATP}}$-triggered GSIS, i.e., ensuring the NSCC-mediated depolarization shift up to $-50 \mathrm{mV}$ [154]. Since this shift is also essential for the net non-amplified GSIS, the cause(s) of the additional triggering and/or increased time of bursts for Cav opening and/or more inhibited $\mathrm{K}_{\mathrm{V}}$ (prolonging spikes) needs to be resolved. Collectively, these events lead to the increased integral $\left[\mathrm{Ca}^{2+}\right] \mathrm{c}$ that determines the surplus insulin secretion.

EPAC2A also directly activates Rim $2 \alpha[205,253]$, thus facilitating SNARE complex formation, relocation (IGV priming) and the subsequent cis to trans conformation change of the major SNARE complex (see above). Moreover, the EPAC2A pathway via Rap2calmodulin kinase II (CaMKII) activates RyR, ensuring $\mathrm{Ca}^{2+}$ efflux from the ER, preceded by $\mathrm{Ca}_{V}$ opening, thus again contributing to the amplification of IGV exocytosis [267]. When there is biased GLP1R (GIPR) signaling via G $\alpha q / 11$, it activates PLC and either IP3IP3R-mediated or PLC plus STIM1-Ora1-TRPC1-mediated $\mathrm{Ca}^{2+}$ efflux from the ER (or TRPC1 migration to the plasma membrane) $[6,119,120]$; and the DAG-PKC pathway that 
phosphorylates and thus activates TRPM4 and 5, so ensuring another essential NSCCmediated shift up to $-50 \mathrm{mV}[6,111]$.

\subsection{Glucagon-Like Peptide 1, GLP-1}

\subsubsection{GLP-1 Amplification of GSIS}

GLP-1 is secreted by enteroendocrine L-cells residing predominantly in the distal ileum and colon upon postprandial stimuli, i.e., by glucose, fatty acids or lipids and proteins $[268,269]$. GLP-1 results from the expression of preproglucagon (Gcg) and via a specific way of cleaving proglucagon (158 amino acids, AA) in L-cells, different from that secreted by pancreatic $\alpha$-cells. Thus, proglucagon is cleaved into glicentin (AA 1-69; containing joined glicentin-related pancreatic polypeptide in AA 1-30, glucagon in AA 33-61 and intervening peptide 1, IP1, in AA 62-69) plus GLP-1 (AA 72-107/108), intervening peptide 2 (AA 111-123) and GLP-2 (AA 126-158) [268]. Glicentin can be further cleaved into glicentin-related pancreatic polypeptide and oxyntomodulin (AA 33-69, i.e., joined glucagon and IP1). The most efficient truncated variants are GLP-1 $1_{(7-37)}$ and its variant GLP-1 $1_{(7-36 a m i d e)}$. The latter is $80 \%$ abundant in humans [270]. These variants are termed simply as GLP-1 in this review. Note that the full peptide GLP-1 $1_{(1-37)}$ is much less efficient at GSIS potentiation [271,272]. In addition, the paracrine GLP-1 signaling acts among the different types of PI cells [271], similarly to the paracrine and endocrine secretion of other hormones. At the systemic level, central control by the brain and nervous system, including GLP-1 secretion in the nucleus tractus solitarii of the brainstem [268], represent the indispensible top level of regulation for insulin secretion, beside local effects of the immune system. GLP- 1 effects related to $\beta$-cell proliferation or apoptosis are beyond the scope of this review.

Only $10-15 \%$ of active GLP-1 likely reaches the pancreas via the circulation system [273]. Thus, typical concentrations of biologically active GLP-1 in human plasma at fasting account for about $2 \mathrm{pmol} / \mathrm{L}$ and at most $10 \mathrm{pmol} / \mathrm{L}$ postprandially [267], peaking 30$60 \mathrm{~min}$ after carbohydrate or protein intake and $120 \mathrm{~min}$ after the ingestion of lipids [274].

\subsubsection{GLP-1 Receptor Signaling}

GLP-1 from the bloodstream acts through its receptor (GLP1R), residing in the plasma membrane of pancreatic $\beta$-cells, which is composed of 463 amino acids in humans and rodents [266]. GLP1R activation stimulates $\mathrm{G} \alpha$ s and $\mathrm{G} \alpha \mathrm{q} / 11$ and recruits $\beta$-arrestin, depending on a biased agonism relatively to different agonists, such as exendin- 4 and oxyntomodulin $[275,276]$. As a scaffold protein, $\beta$-arrestin facilitates the signaling via G $\alpha$ s to cAMP, but also to CREB [276], extracellular regulated kinase ERK1/2 [277] and insulin receptor substrate 2 (IRS-2), its effects promoting $\beta$-cell growth, differentiation and maintenance [276]. The stimulation of G $\alpha$ s leads to the initiation of PKA via enhanced cAMP [278], which also stimulates the EPAC2A pathways [279]. Continuous cAMP production and the partial potentiation of GSIS were found even for the internalized GLP1R [280].

The PKA pathway provides a surplus intracellular $\mathrm{Ca}^{2+}$ above that of the net GSIS (i.e., without any receptor stimulation) [281]. Phosphorylation and so deactivation of $\mathrm{K}_{\mathrm{V}}$ channels prolongs the overall $\mathrm{Ca}^{2+}$ stimulation signals. This leads to somewhat lower frequencies of $\mathrm{Ca}^{2+}$ oscillations, but with each spike lasting longer [282]. In principle, this phenomenon may also potentiate the 2nd phase of GSIS. In contrast, the PKA-mediated phosphorylation of snapin engages a higher interaction of SNAP- 25 with synaptogamins of IGVs, reportedly potentiating the 1st GSIS phase [194,195].

Any phase of insulin secretion within its basic triggering, and its potentiation mechanism by GLP1R signaling, should be dependent on NSCCs. Hence, one can consider that the observed simultaneous stimulation of TRPM2 channels by the EPAC2A pathway [154] is the prerequisite in both. It remains to be resolved whether the TRPM2 stimulation by EPAC activates a greater population of these or other NSCC channels compared to the absence of the GLP1R pathway, i.e., to the basic GSIS. As was mentioned above, the EPAC2A pathway simultaneously promotes the docking and priming of IGVs by al- 
lowing Rab3A interaction with Rim $2 \alpha$ [205]; and hypothetically also the interaction of EPAC2-Rim $2 \alpha$-Picollo trimers with Rab3A enables more intensive IGV exocytosis [268]. If a biased GLP1R-G $\alpha$ q/11 stimulation occurs, promotion should also be superimposed by the $\mathrm{Ca}^{2+}$-induced (meaning by $\mathrm{Ca}_{\mathrm{V}}$ opening) RyR-mediated and or IP3R-mediated $\mathrm{Ca}^{2+}$ release from the ER [279]; plus activating the phosphorylation of TRPM4 and TRPM5 via the DAG-PKC pathway [219].

However, note that the above description represents a simplification, since it is necessary to understand the detailed contribution of GLP1R signaling to the overall dynamics of $\mathrm{Ca}_{\mathrm{V}}$ opening (action potential spikes), i.e., to quantify the frequencies, bursting time, and interburst lag time; plus the contribution to $\left[\mathrm{Ca}^{2+}\right]_{\mathrm{c}}$ oscillations of superimposed waves originating due to the oscillating $\mathrm{Ca}^{2+}$ release from the stores, such as ER, IGVs and/or mitochondria. These events were indeed analyzed by conducting simultaneous electrophysiological and $\mathrm{Ca}^{2+}$ oscillation monitoring, the latter using a proper $\mathrm{Ca}^{2+}$ fluorescent probe(s) at $2 \mathrm{mM}$ glucose but with $200 \mu \mathrm{M}$ tolbutamide blocking $\mathrm{K}_{\text {ATP }}$ [282]. Under these conditions, the GLP-1 analog liraglutide apparently decreased the frequency of action potential spikes, which however were individually wider, as with ablated Kv2.1 channels. So, this most likely reflected the PKA-mediated inactivation of Kv2.1 channels. Independently of liraglutide, each action potential spike corresponded to a triangular peak of the cytosolic $\mathrm{Ca}^{2+}$ rise. As a result, the overall integrated $\left[\mathrm{Ca}^{2+}\right]_{\mathrm{c}}$ increase over the elapsed time is much higher than upon the net GSIS, which is not amplified by GLP-1.

Earlier experiments with $7.7 \mathrm{mM}$ glucose and using GLP- $1_{(7-36)}$ amide (preproglucagon 78-107) also clearly indicated an increased duration of active and silent electrical activity [283]. Again, the time-width of $\mathrm{Ca}^{2+}$ increased from 2 to about $5 \mathrm{~s}$ with liraglutide [282]. The relative duration vs. the active phase of $\mathrm{Ca}^{2+}$ spikes was $10 \%$ at $4 \mathrm{mM}, 50 \%$ at $7 \mathrm{mM}$ and $80 \%$ at $9 \mathrm{mM}$ glucose [284]. This delayed decay of the $\mathrm{Ca}^{2+}$ responses is not only given by the prolonged action potential spikes, but also stems from the $\mathrm{Ca}^{2+}$ released from the intracellular stores, namely the ER. The intermittent responses of proteins of the exocytotic machinery, namely the frequency of formation of SNARE complexes and resulting pulsatile IGV exocytosis, was elegantly monitored by the ATP-activated currents conducted by the overexpressed P2X2 cation channels [285]. This was made possible by the fact that IGVs contain a high ATP concentration.

\subsubsection{Two Phases of Insulin Secretion vs. GLP-1 Potentiation}

Both cAMP and 8-Br-cAMP increased the frequency of fusion events, i.e., the fusion of insulin granules to the plasma membrane in both phases of GSIS, in experiments using TIRF while simulating GLP-1 effects [85]. EPAC2A interacts with the small G protein Rap1, affecting its conformation so that it releases the catalytic region, which subsequently binds and thus activates another $\mathrm{G}$ protein Rap113. In EPAC2A knockout mice, most of the potentiation of the 1st GSIS phase vanished [85]. Thus, one can speculate that the 2nd phase amplification can be predominantly upregulated due to the PKA pathway.

\subsubsection{Does GLP-1 Stimulate Insulin Secretion at Low Glucose?}

The traditional view has considered that incretin signaling does not stimulate insulin release under low glucose conditions [271,272]. However, in experiments mimicking the physiological situation "low glucose" should be equal to fasting glycemia. Under these conditions, however, GLP-1 in supraphysiological concentrations did initiate insulin secretion. The controversies are therefore over whether reported experiments reflect the physiologically occurring postprandial GLP-1 $1_{(7-37)}$ concentration in islet capillaries of rodents (GLP-1 (7-36amide) in humans).

It has been observed that the intermediate glucose concentration, such as $6 \mathrm{mM}$, allows certain insulin secretion in rats as the response to GLP- $1_{(7-37)}$, but, in contrast, at $<2.6 \mathrm{mM}$ glucose GLP-1 $1_{(7-37)}$ does not stimulate insulin secretion [271,272]. Note that $6 \mathrm{mM}$ glucose approaches to the fasting glycemia values in rats. As described above, the store-release of $\mathrm{Ca}^{2+}$ should depend on the Cav opening, but this comes automatically, since both GLP1R 
signaling branches, PKA and EPAC2 pathways, prolong durations of Cav opening. PKA does this directly by phosphorylating $\mathrm{Ca}_{\mathrm{V}}$ (besides $\mathrm{K}_{\mathrm{V}}$ and $\mathrm{K}_{\mathrm{ATP}}$ ) and EPAC2 provides the basic or hypothetically even the amplified TRPM2 action. Theoretically, the GLP1R signaling can be considered to be independent of the high glucose concentrations, since the basic shift to $-50 \mathrm{mV}$ is provided by the EPAC2-activated TRPM2 [154] and since the PKA phosphorylation of $\mathrm{K}_{\mathrm{ATP}}, \mathrm{Kv}$ and especially of $\mathrm{Ca}_{\mathrm{V}}$ channels might provide the triggering. If all these considerations turned out to be plausible, we must ask the question of whether all the proteins of IGV exocytotic machinery could function at the $\mathrm{Ca}^{2+}$ levels established at these low or intermediate glucose concentrations. Indeed, certain synaptogamins were reported to be independent of $\mathrm{Ca}^{2+}[230]$.

\subsection{Glucagon Inhibitory Peptide, GIP}

GIP acts similarly to GLP-1 [286]. GIP is secreted by the K-cells in the proximal gut upon a stimulus when chyme enters the duodenum. Similarly to GLP-1, it has been reported that the GIP-amplification of GSIS is dependent on higher glucose levels, cAMP production due to the GIP receptor (GIPR) signaling is not $[287,288]$. The pathways downstream of GIPR are thought to be identical to those for GLP-1. For example, Rim $2 \alpha$ knockout mice exhibited impaired GIP amplification of GSIS, indicating the absence of the GIPR-cAMP-EPAC2-enhancement of IGV exocytosis [205]. Similarly to GLPR, GIPR long-term signaling exhibits profound prosurvival effects for pancreatic $\beta$-cells [289].

\section{Role of Redox Shuttles upon GSIS}

\subsection{Redox Shuttles Exporting Redox Equivalents to the Cytosol of Pancreatic $\beta$-Cells}

Even without knowledge of NOX4 participation in GSIS, it has long been known that an increase in cytosolic NADPH facilitates insulin secretion responding to glucose $[16,37,290-293]$. The three major metabolic shuttles were revealed, which are activated at higher glucose and export reducing equivalents of NADH from the mitochondrial matrix to the cytosol during GSIS [290]. Since the major metabolite influx into mitochondria upon increasing glucose is given by pyruvate, all three shuttles are related to pyruvate [294]. One can recognize the pyruvate/malate, pyruvate/citrate and pyruvate/isocitrate shuttles (Figure 6).

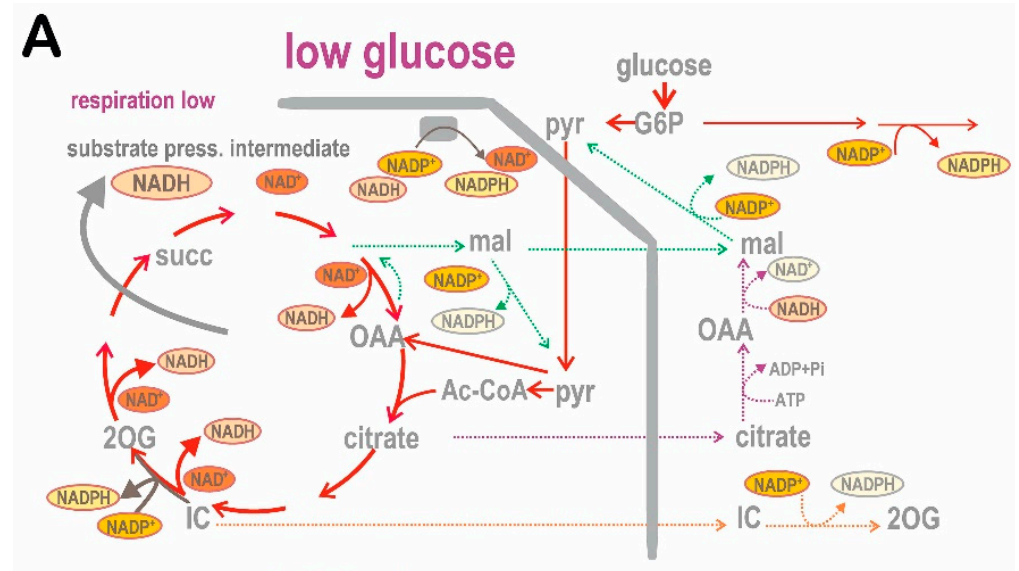

Figure 6. Cont. 


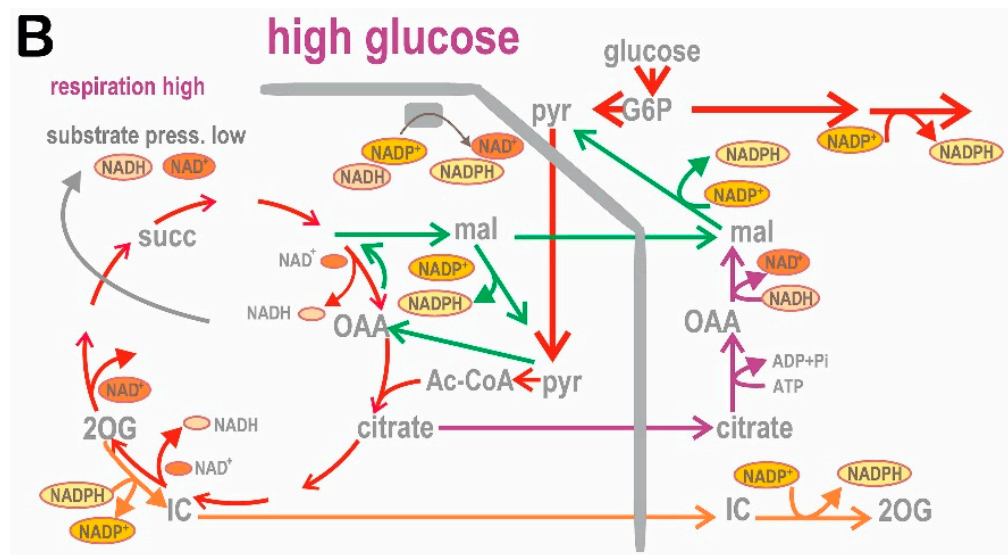

Figure 6. Redox shuttles-(A) operate at minimum in low glucose conditions, but (B) at maximum in the high glucose conditions. The key enzyme for the pyruvate/malate shuttle (green arrows) is the pyruvate carboxylase (PC) allowing the reverse malate dehydrogenase (MDH) reaction and following the export of malate (Mal) to the cytosol, where malic enzyme 1 (ME1) forms NADPH. As a result, instead of NADH, which would otherwise be made by $\mathrm{MDH}$, a cytosolic NAPDH is formed. Similar consequences result from the operation of the pyruvate/citrate shuttle and pyruvate/isocitrate shuttle ("IC", isocitrate). The latter involves NADPH-dependent reductive carboxylation by isocitrate dehydrogenase 2 (IDH2) in the mitochondrial matrix, followed by the export of the formed IC, and oxidative decarboxylation by the cytosolic IDH1, forming NADPH, which again contributes to the elevated cytosolic NADPH pool. Note that the operation of these shuttles under conditions of high glucose excludes the operation of the malate/aspartate, which requires exactly the opposite 2-oxoglutarate fluxes.

\subsubsection{Pyruvate/Malate Redox Shuttle}

The reaction sequence for the pyruvate/malate redox shuttle bypasses the regular pyruvate entry into the Krebs cycle via pyruvate dehydrogenase (PDH). Instead, the pyruvate carboxylase reaction takes place. The resulting oxaloacetate can either increase the turnover of the forward Krebs cycle or may be converted by the reverse reaction of malate dehydrogenase (MDH) to malate. This counter-Krebs cycle flux is the key element in the pyruvate/malate shuttle. Malate can be subsequently exported to the cytosol, either by the 2-oxoglutarate carrier (SLC25A11) [292], being exchanged for 2OG import into the mitochondrial matrix. The exported malate aids the cytosolic malate pool. This pool can be consumed by the malic enzyme 1 (ME1) reaction, driven by $\mathrm{NADP}^{+}$, so being one of the cytosolic sources of NAPDH $[295,296]$. The proper reaction direction within the $\beta$-cell cytosol is driven by an immediate consumption of pyruvate by mitochondria. So, the pyruvate influx into the matrix, enabled by the pyruvate carrier (MPC1 and MPC2; providing pyruvate- $\mathrm{H}^{+}$symport) both begins and ends the pyruvate/malate shuttle.

\subsubsection{Pyruvate/Citrate Redox Shuttle}

The pyruvate/citrate shuttle exists due to citrate export from the matrix, while the Krebs cycle is truncated just after the reaction of citrate synthase [297]. ${ }^{13} \mathrm{C}$-tracing studies showed that the predominant amount of citrate originates from glucose-derived acetyl-CoA in INS/1 832/13 cells, indicating that a quite high fraction of citrate escapes the Krebs cycle [62]. Such an export exists frequently in cancer cells. However, it is also of great importance for GSIS. Citrate is exported by the citrate carrier (SLC25A1), enabling citrate antiport with malate. Note that the malate cycling can actually take place in conjunction with the pyruvate/malate redox shuttle. In the cytosol, citrate is split by the ATP-citrate lyase (ACL) in the reaction with coenzyme A $(\mathrm{CoA})$ into oxaloacetate and acetyl-CoA (AcCoA). Cytosolic MDH converts oxaloacetate into malate and also this shuttle uses ME1 and a pyruvate carrier to produce and import pyruvate into the matrix, respectively. In parallel, ME1 supplies NADPH for NOX4-mediated redox signaling. Note that the ACL reaction may be substituted by the acetoacetate pathway (see Section 4.2.5), so that at low 
glucose either one maintains the short-chain acyl-CoA levels in the cytosol, but at GSIS the acetoacetate pathway predominates [298].

\subsubsection{Pyruvate/Isocitrate Redox Shuttle}

The pyruvate/isocitrate shuttle is based on the reductive carboxylation reaction of isocitrate dehydrogenase 2 (IDH2), residing in the mitochondrial matrix. This is a reverse reaction compared to the typical $\mathrm{NADP}^{+}$-driven isocitrate oxidation, and produces $2 \mathrm{OG}$ and NADPH. This oxidative IDH2 reaction serves as a parallel bypass of the regular IDH3 reaction of the Krebs cycle. However, instead of NADH produced by IDH3, IDH2 forms NADPH.

Upon a higher glucose intake, the forward (oxidative) mode is reversed to the counterKrebs cycle direction. This reductive carboxylation mode requires $\mathrm{CO}_{2}$ and enables the operation of the pyruvate/citrate cycle [37]. At the same time, the aconitase reaction and isocitrate formation is slow, thus allowing the reverse IDH2 reaction, despite the $\mathrm{CO}_{2}$ requirement. The citrate carrier then readily exports isocitrate to the cytosol. Subsequently, the cytosolic $\mathrm{NADP}^{+}$-driven IDH1-mediated oxidation of isocitrate takes place, so $2 \mathrm{OG}$ and NADPH are produced [295]. IDH1 within this shuttle is an important source of the cytosolic NADPH pool and contributes to its increase upon GSIS [293]. 2OG enters the matrix by being exchanged for malate via by the oxoglutarate carrier. 2OG then either enters the regular Krebs cycle 2OG-dehydrogenase reaction; or 2OG completes this cycle, again being the substrate of IDH2-mediated reductive carboxylation.

\subsection{Specific Metabolism at Insulin-Non-Stimulating vs. Insulin-Stimulating Glucose in Pancreatic $\beta$-Cells}

\subsubsection{NADPH and NADH Homeostasis in Pancreatic $\beta$-Cells}

The above detailed description of redox shuttles considered their functioning at high, insulin-stimulating glucose, i.e., at maximum metabolic turnover and maximum OXPHOS. As a result, these shuttles do not allow maximum NADH to be produced in the mitochondrial matrix, but instead, more NADPH is produced in the cell cytosol. In fact, this epresents a transfer of redox equivalents from the matrix to the cell cytosol. The shuttles thus serve as an independent NADPH source feeding NOX4 to initiate redox signaling that enables $\mathrm{K}_{\mathrm{ATP}}$ closing (and/or TRPM2 opening), resulting in insulin secretion.

For example, as part of the pyruvate/malate redox shuttle, mitochondrial MDH produces less NADH, than with the $100 \%$ forward reaction. However instead, malate produced by the reverse MDH reaction is used by ME1 to form NADPH in the cytosol. Similarly, if a portion of the isocitrate is not converted by IDH3 to produce NADH, but instead the reverse (reductive carbonylation) IDH2 reaction occurs, while isocitrate exported into the cytosol evokes NADPH formation by IDH1. Again, instead of the production of one NADH molecule in the matrix, one NADPH molecule is formed in the cytosol. In order to facilitate the exocytosis of IGVs by glutamate, the reported glutamate dehydrogenase mode in the matrix should involve the reaction producing glutamate and $\mathrm{NAD}^{+}$, while consuming 2OG, ammonium and NADH [66]. One can predict that this reaction will also contribute to the decreasing NADH in the matrix.

We predicted and verified experimentally [37] that the decreasing matrix NADH during the concomitantly increasing $\mathrm{NAD}^{+}$at the simultaneous maximum respiration leads to a decreased matrix substrate pressure $S P$, defined as the NADH/NAD ${ }^{+}$ratio in the mitochondrial matrix. Using inhibitors of superoxide formation at specific RC sites, we have provided evidence that even slightly decreasing $S P$ reduces superoxide formation at the flavin site $\mathrm{I}_{\mathrm{F}}$ of RC complex I [37]. Note, however, that a typical matrix concentration of $\mathrm{NAD}^{+}$was estimated to be two orders of magnitude higher than the NADH concentration. In HeLa cells, for example, $800 \mu \mathrm{M} \mathrm{NAD}^{+}$was found besides $5 \mu \mathrm{M}$ NADH [299]. For pancreatic $\beta$-cells, set to a faster respiration upon GSIS, this means that each NADH molecule formed by the respective matrix dehydrogenases is immediately consumed and used by complex I. 


\subsubsection{Nicotine Nucleotide Translocase in Pancreatic $\beta$-Cells}

Somewhat substantial concerns were raised regarding the mitochondrial nicotine nucleotide transhydrogenase (NNT) in pancreatic $\beta$-cells. In its forward mode, NNT consumes $\Delta \mathrm{p}$ by allowing $\mathrm{H}^{+}$influx into the matrix, which is coupled with the conversion of $\mathrm{NADP}^{+}$to NADPH with simultaneous NADH consumption, thus producing $\mathrm{NAD}^{+}$[300]. Note that NNT acts downstream of the redox shuttles, so it should not influence them. However at low glucose, NNT might act in the reverse mode [301], in which it functions as a proton pump, enhancing $\triangle \mathrm{p}$ and consuming $\mathrm{NADPH}$ and $\mathrm{NAD}^{+}$, while producing $\mathrm{NADP}^{+}$and NADH. At low glucose, the respiration and ATP synthesis are at slower rates; establishing a lower $\Delta \mathrm{p}$. Therefore, $\mathrm{H}^{+}$pumping mediated by NNT against an intermediate $\Delta \mathrm{p}$ would be hypothetically easier than at high glucose against the higher $\Delta \mathrm{p}$. The existence of the NNT reverse mode was suggested, based on different types of experiments with mouse pancreatic $\beta$-cells [301]. To our knowledge, the direct observation of the $\mathrm{H}^{+}$flux direction by NNT has never been attempted. Our preliminary data concerning the comparison of experimentally determined $\Delta \Psi_{\mathrm{m}}$, using fluorescent indicators of membrane potential, showed instead increases in $\Delta \Psi_{\mathrm{m}}$ in NNT-silenced INS-1E cells [37]. This suggests that NNT acts in the forward mode, producing NADPH.

Experiments relying on a comparison of C57BL/6J vs. C57BL6/ $\mathrm{N}$ mice were also controversial. In C57BL/6J mice, an in-frame five-exon deletion in the Nnt gene spontaneously occurred, removing exons 7-11 and leading to a complete absence of the NNT protein [302-304]. It was reported that there is a high blockage of GSIS in the C57BL6/J mouse strain, which others and we never observed [4,305]. The discrepancy may originate from the fact that initially only the quantitative trait loci (QTLs) were identified and correlated with deletions in the Nnt gene. The subsequent verification using artificial Nnt expression could be inconclusive, since the Nnt expression per se could enhance insulin secretion, leading to its apparent suppression in C57BL6/J mice [305].

\subsubsection{Mitochondrial Matrix NADPH Homeostasis in Pancreatic $\beta$-Cells}

It has also been observed that matrix NADPH decreases with operating redox shuttles, specifically due to the operation of the pyruvate/isocitrate shuttle. Such an NADPH decrease was confirmed experimentally in INS-1E cells [37]. Hence, even if the NADPH formation was increased upon GSIS by the activated matrix malic enzyme ME3 and by the increasing forward ( $\Delta \mathrm{p}$ consuming) mode of $\mathrm{NNT}$, the latter two sources cannot exceed the NADPH consumption by the mitochondrial IDH2. ME3 was reported to form pyruvate and $\mathrm{NADPH}$ from malate and $\mathrm{NADP}^{+}$in the mitochondrial matrix [306]. The acute NADPH increase has a disadvantage in that it decreases the reduced glutathione (GSH) and increases its oxidized form (GSSH) in the mitochondrial matrix. One can view the simultaneous existence of redox shuttles with decreasing GSSH as a fair trade for GSIS, lying in the preferable provision of cytosolic NADPH elevations prior to the maintenance (or at the expense of) mitochondrial matrix GSH.

\subsubsection{Relationships to the Malate/Aspartate Shuttle}

A major role has been suggested for the malate/aspartate shuttle in pancreatic $\beta$ cells [279,307]. However, one must recognize that the active malate/aspartate shuttle operation upon GSIS excludes the operation of the pyruvate/malate and pyruvate/isocitrate shuttles [37], the existence of which was documented by numerous experiments [16,275-278]. The malate/aspartate shuttle could not effectively function under high glucose conditions, since the 2-oxoglutarate carrier would have to translocate malate in the opposite direction to the malate export, which is active during the pyruvate/malate and pyruvate/isocitrate redox shuttles. Additionally, as part of the malate/aspartate shuttle, glutamate must be imported to the mitochondrial matrix, coupled to the aspartate efflux. However, several laboratories reported that matrix-formed glutamate is also exported to the $\beta$-cell cytosol to facilitate IGV maturation and exocytosis [306,308-310]. This again requires the opposite direction of fluxes. At least glutamate is beneficial for the GLP-1 amplification of GSIS [67,311]. 
We found that if there is any contribution of the malate/aspartate shuttle, it must act at low non-stimulating glucose [37]. Previously, the support for the existence of the malate/aspartate shuttle was considered to stem from the essential requirement of transaminases (aminotransferases) and two isoforms of aspartate/glutamate antiport carrier: SLC25A12, i.e., Aralar or AGC1 [312,313]; and SLC25A13, i.e., AGC2. Metabolomics studies showed that the total cell aspartate is diminished at the initiation of GSIS, while aconitate, citrate, isocitrate, malate or fumarate rose instantly, and elevations of 2-OG or succinate were delayed by $15 \mathrm{~min}$ [38]. However, elevations of metabolites can originate from the delayed reaction consuming that particular metabolite, such as acting in the malate/aspartate shuttle.

\subsubsection{Acetoacetate and $\beta$-Hydroxybutyrate in Pancreatic $\beta$-Cells}

The $\beta$-hydroxybutyrate dehydrogenase $(\beta-\mathrm{OHBDH})$, which is found exclusively in the mitochondrial matrix, was also studied in pancreatic $\beta$-cells $[116,314]$. The previous consensus for hepatocytes considered that the ratio of $\beta$-hydroxybutyrate to acetoacetate reflects the established matrix $\mathrm{NAD}^{+} / \mathrm{NADH}$ ratio $(1 / S P)$ [314]. However, since the estimated order of magnitude for $1 / S P$ is $>100$ [299], such a ratio of $\beta$-hydroxybutyrate to acetoacetate is unlikely. Additionally, the $\mathrm{NADH}$-driven $\beta-\mathrm{OHBDH}$ reaction converting acetoacetate to $\beta$-hydroxybutyrate and $\mathrm{NAD}^{+}$is not the only one involving acetoacetate in pancreatic $\beta$-cells $[116,314]$. Acetoacetate is also exported to the cytosol of $\beta$-cells, where it reportedly facilitates insulin secretion via forming various acyl-CoA derivatives [116]. Additionally, $\beta$-OHBDH cannot be formed in the cytosol of rodents, but can be formed in the $\beta$-cell cytosol in humans (for further details see https: / / www.brenda-enzymes.org / enzyme.php?ecno=1.1.1.30). All these reactions influence redox homeostasis.

Under high glucose conditions, the sufficient succinate load enables its interconversion by succinyl-CoA:3-ketoacid-CoA transferase (SCoA:3oxoAcCoAT) with acetoacetylCoA (AcAcCoA) into succinyl-CoA (Succ-CoA) and acetoacetate [298]. This can also be formed by leucine metabolism, within a series of reaction of $\beta$-like oxidation leading to hydroxymethyl-glutaryl-CoA (HMG-CoA), which is split by HMG-CoA lyase (HMGCoAL). From the acetoacetate pool in the matrix, acetoacetate can be easily transported to the cytosol or may be converted by $\beta-\mathrm{OHBDH}$ to $\beta$-hydroxybutyrate at the expense of $\mathrm{NADH}$, thus forming $\mathrm{NAD}^{+}$. Note that distinct enzyme isoforms convert two molecules of acetyl-CoA (Ac-CoA) into CoA and AcAcCoA in the mitochondrial matrix (acetyl-CoA acetyltransferase, ACAT1; and acetyl-CoA acyltransferase, ACAA2) and cytosol (acetylCoA acetyltransferase, ACAT2; and acetyl-CoA acyltransferase, ACAA1). Cytosolic acetylCoA was suggested to facilitate the acetylation of proteins, which might speculatively enhance GSIS [304,305]. In conclusion, acetoacetate and acyl-CoA metabolism should be studied further, not only revealing metabolomics data, but also in order to elucidate in detail whether they amplify/stimulate insulin secretion or not.

\subsubsection{Phosphoenolpyruvate Shuttle and the Role of Pyruvate Kinases}

Yet another cycle may be important for pancreatic $\beta$-cells in the resting regime, when no insulin secretion takes place. This is the so-called phosphoenolpyruvate (PEP) cycle, which is cataplerotic in character. It begins when the mitochondrial matrix oxaloacetate is converted to PEP by the mitochondrial PEP-carboxykinase (PEPCK2). PEP is subsequently exported to the cytosol by the citrate carrier (SLC25A1). There are several cytosolic isoforms of pyruvate kinase (PK) in $\beta$-cells, such as the constituent M1 isoform and recruitable M2 and L [315]. PKs convert cytosolic PEP to pyruvate. This reaction is coupled to ATP formation by the "substrate" phosphorylation of ADP and is the regular part of glycolysis. That is why at high glucose, glucose metabolism is highly competitive and the PEP cycle should contribute to a lesser extent. Nevertheless, under both low and high glucose conditions, pyruvate then enters mitochondria, where it is metabolized either by PDH or PC. The PC flux completes the PEP cycle by the conversion of pyruvate to oxaloacetate. 
Since fructose 1,6-bisphosphate allosterically activates PKM2 and PKL, their reactions supply the ATP required for $\mathrm{K}_{\text {ATP }}$ closure. This was recently derived from the results of patch-clamp experiments in the excision mode combined with PK activation by a small molecule activator [100]. The PEP-cycle was reportedly switched on/off in the $\beta$-cell responses to intermediate $9 \mathrm{mM}$ glucose, when $V \mathrm{p}$ and/or $\left[\mathrm{Ca}^{2+}\right]_{\mathrm{c}}$ bursts phases are intermittent with the interburst phases (cf. Figure 3). The decreased cytosolic ATP / ADPratio was explained on the basis of the PEP cycle providing ATP by PKs, which should be independent of OXPHOS. Prior to glucose elevation, or after termination of the burst phase at $9 \mathrm{mM}$ glucose, lower ATP levels were observed, which set $\mathrm{K}_{\mathrm{ATP}}$-channels open. Under these conditions, the control strength of the PEP cycle is high. When OXPHOS continues at this intermediate $9 \mathrm{mM}$ glucose concentration, thus continuing the elevation of ATP, the control strength of the Krebs cycle overcomes that of the PEP cycle and it possesses less control strength. As a result, at this moment the burst phase begins at $9 \mathrm{mM}$ glucose. Additionally, a PKM2 and PKL activator failed to improve GSIS in PEPCK2-knockout mice, demonstrating the important contribution of the PEP cycle [316].

\section{Mechanism of Insulin Secretion Stimulated by Branched-Chain Ketoacids}

A postprandial response of insulin secretion is also produced by substances other than glucose, which induce the secretion of insulin. These substances are termed secretagogues in general. One important type of secretagogue is branched-chain ketoacids (BCKAs). These are metabolites of branched-chain amino acids (BCAAs). BCKAs involve three major metabolites, 2-ketoisocaproate (KIC; leucine metabolite) [317], 2-ketoisovalerate (KIV; valine metabolite) and 2-ketoisomethylvalerate (KMV; isoleucine metabolite) [318-322]. We will discuss mechanisms leading to redox signaling upon BCKA metabolism [323].

\subsection{Superoxide Formation and Subsequent Redox Signaling upon $\beta$-Like Oxidation of Branched-Chain Ketoacids}

5.1.1. Electron Transfer Flavoprotein: Ubiquinone Oxidoreductase (ETFQOR) as the Key Factor

The metabolism of BCKAs is initiated in the mitochondrial matrix by the BCKA dehydrogenase complex (BCKDH; not present in the cytosol), following a similar sequence of reactions to the $\beta$-oxidation of fatty acids, termed $\beta$-like oxidation. The BCKDH reaction [323] within the $\beta$-like oxidation leads to the elevated formation of superoxide in the mitochondrial matrix. Indeed, the silencing of the BCKDH E1 $\alpha$ subunit prevented both the KIC-induced matrix superoxide release and insulin secretion stimulated with KIC in INS-1E cells [4].

The BCKDH complexes provide reactions of isovaleryldehydrogenase (IVD, EC 1.3.99.10) and similar dehydrogenases of isobutyryl-CoA and methyl-isobutyryl-CoA, all having FAD as a cofactor, converting it to $\mathrm{FADH}_{2}$. Subsequently, the electron-transfer flavoprotein (ETF) accepts electrons from BCKDH as one electron carrier and diffuses up to the electron-transfer flavoprotein: ubiquinone oxidoreductase (ETFQOR) located in the matrix surface of IMM [324,325].

Superoxide formation may originate due to the transfer of electrons from the ETFQOR to the mitochondrial ubiquinone pool [325]. Either an electron leak from flavin to the oxygen leading to radical pair formation produces superoxide within ETFQOR itself [326]; or since ETFQOR needs ubiquinon (Q), reduced after accepting two electrons from the ETF to ubiquinol $\left(\mathrm{QH}_{2}\right)$, it effectively competes with the mitochondrial respiratory chain complex I. Since $\mathrm{QH}_{2}$ binds to the complex I Q-binding site, it allows a feedback inhibition of the ongoing $\mathrm{Q}$ reduction to $\mathrm{QH}_{2}$ within complex I. This increases the mitochondrial superoxide formation at the $\mathrm{I}_{\mathrm{Q}}$ site of superoxide formation [312]. In other words, the electron transfer within complex I is effectively retarded in this way and therefore superoxide formation takes place in its $\mathrm{I}_{\mathrm{Q}}$ site. Brand and colleagues suggested that dehydrogenases alone can also produce superoxide [327]. However, this is yet to be confirmed or excluded for BCKDH. Additionally, superoxide may be formed by complex I and complex III of the mitochondrial respiratory chain due to the resulting excessive acetyl-CoA en- 
try (propionyl-CoA entry for KIV; through methylmalonyl and succinyl-CoA) into the Krebs cycle. Moreover acetoacetate, being one of the final products of leucine metabolism, could influence the established redox homeostasis.

\subsubsection{Redox Signaling upon $\beta$-Like Oxidation of Branched-Chain Ketoacids}

Independently of the molecular mechanism, BCKA metabolism leads to increased mitochondrial superoxide formation. After conversion to $\mathrm{H}_{2} \mathrm{O}_{2}$ by the matrix MnSOD and the intermembrane space $\mathrm{CuZnSOD}$, the ongoing $\mathrm{H}_{2} \mathrm{O}_{2}$ efflux from mitochondria can be regarded as redox signaling. We have clearly demonstrated that the absence of such redox signaling, otherwise stimulated with BCKAs, leads to a blockage of insulin secretion, for example in the presence of the mitochondrial matrix-targeted antioxidants SkQ1 [4]. Likewise, the silencing of BCKDH led to the inhibition of insulin secretion stimulated with BCKAs.

In summary, superoxide formed in an enhanced manner upon $\beta$-like oxidation is converted to elevated $\mathrm{H}_{2} \mathrm{O}_{2}$. The emanation of $\mathrm{H}_{2} \mathrm{O}_{2}$ from the matrix to targets, located e.g., in the plasma membrane, subsequently substantiates the mitochondrial retrograde redox signaling. The targets providing insulin secretion could be $\mathrm{K}_{\mathrm{ATP}}$, TRPM2 or both, as with GSIS. Simultaneously, the metabolism of BCKA [328,329], followed by OXPHOS, leads to the concomitantly elevated ATP (Figure 7). Note also that the redox signaling can take place either by direct diffusion or hypothetically also via redox relay systems (see Section 7).

\subsection{Metabolism of Branched-Chain Ketoacids}

\subsubsection{Branched-Chain Amino Acids as Precursors of Branched-Chain Ketoacids}

Leucine, valine and isoleucine are imported into $\beta$-cells by various transporters, predominantly by LAT1/SLC7A5 [330]. BCAA metabolism proceeds in mitochondria, since there is no branched-chain amino acid aminotransferase (BCAT) in the $\beta$-cell cytosol [328,329] (Figure 7). Additionally, the nature of BCAA import into the mitochondrial matrix is unknown. BCKAs are most likely imported into the mitochondria of pancreatic $\beta$-cells by the carnitine carrier (SLC25A20). The mitochondrial matrix BCAT with a cofactor (pyridoxal-5'-phosphate) reversibly converts BCAAs and $2 \mathrm{OG}$ to BCKA and glutamate [328,329]. The resulting BCKAs are degraded by the mitochondrial branched-chain ketoacid dehydrogenase (BCKDH) complex [323,331]. This reaction is coupled to the conversion of CoA to proper BCKA-CoAs.

\subsection{2. $\beta$-Like Oxidation of Branched-Chain Ketoacids}

$\mathrm{BCKDH}$ forms isovaleryl-CoA, isobutyryl-CoA and methyl-isobutyryl-CoA from KIC, KIV and KMV, respectively. The next series of reactions is similar to the mitochondrial $\beta$-oxidation of fatty acids, hence is termed $\beta$-like oxidation. The sequence of reactions for KIC is given by the enzymes in the following order: isovaleryl-CoA dehydrogenase (IVD, EC 1.3.99.10), methylcrotonyl-CoA carboxylase (MCC), methyl-glutoconyl-CoA hydratase (MGCoAH) and 3-hydroxy- 3-methylglutaryl-CoA lyase (HMGCoAL). The end products are acetyl-CoA and acetoacetate. Similarly as for pyruvate metabolism via PDH, the common end product acetyl-CoA drives the Krebs cycle. This metabolic acceleration itself may increase mitochondrial superoxide formation.

The BCKDH complex consists of three catalytic components [332]: $\alpha$-ketoacid dehydrogenase (E1), dihydrolipoyl transacylase (E2) and dihydrolipoamide dehydrogenase (E3). In total 24 copies of E2 are arranged in octahedral symmetry and form the core [333]. Twelve E1 $\alpha 2 \beta 2$ tetramers are non-covalently linked to the core as well as six E3 homodimers. The inner-core domain catalyzes the acyltransferase reaction. The BCKDH complex is also indirectly activated by $\mathrm{Ca}^{2+}$ via a mechanism that cancels the inhibitory phosphorylation of the E1 subunit. E1P-phosphatase dephosphorylates the E1 subunit, while phosphorylation is enabled by the BCKDH-E1-kinase that is in turn inhibited by the cofactor thiaminepyrophosphate. The cofactor-mediated kinase inhibition is strengthened by $\mathrm{Ca}^{2+}$, hence $\mathrm{Ca}^{2+}$ activates $\mathrm{BCKDH}[334]$. 


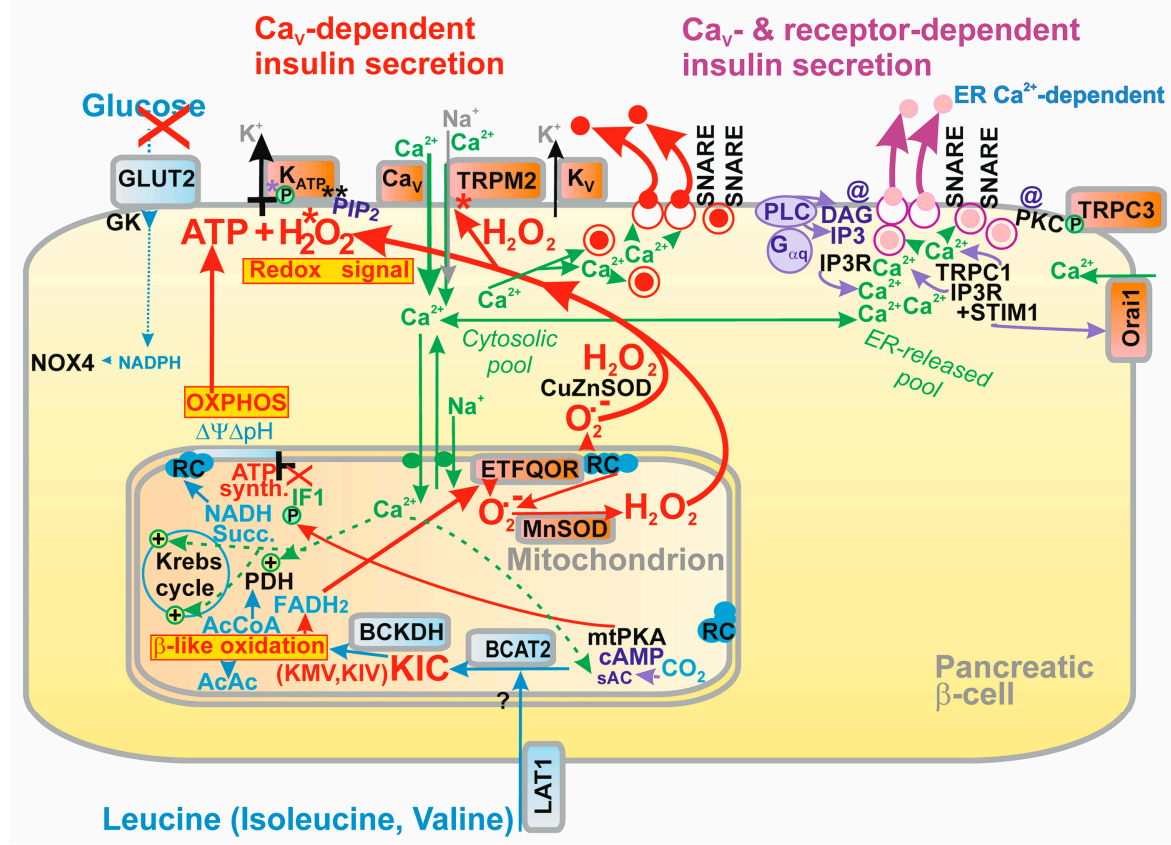

Figure 7. The mechanism of branched-chain keto acid-stimulated insulin secretion involves redox signaling of mitochondrial origin. 2-ketoisocaproate (KIC), 2-ketomethylvalerate (KMV) and 2ketoisovalerate (KIV) resulting from leucine, isoleucine and valine (imported into cells by the plasma membrane LAT1 transporter), respectively, due to the branched-chain aminotransferase reaction in the mitochondrial matrix (BCAT2), are metabolized by branched-chain ketoacid dehydrogenase $(\mathrm{BCKDH})$, both existing exclusively in the mitochondria of $\beta$-cells. A series of reaction of $\beta$-like oxidation begins at the dehydrogenase step, yielding $\mathrm{FADH}_{2}$. This dehydrogenase cofactor is reoxidized by the ETF, as a single electron carrier, from which ETFQOR accepts electrons and converts $\mathrm{Q}$ to $\mathrm{QH}_{2}$. As a side reaction, superoxide is formed by the respiratory chain or even within the ETFQR. In the mitochondrial matrix, superoxide is transformed to $\mathrm{H}_{2} \mathrm{O}_{2}$ by MnSOD, whereas by CuZnSOD in the intermembrane space and cytosol. The elevated mitochondrial/cytosolic $\mathrm{H}_{2} \mathrm{O}_{2}$ substitutes the redox signaling of the NOX4 origin. Consequently, such redox signaling, together with elevated ATP, sets the triggering conditions for insulin secretion. Either both $\mathrm{H}_{2} \mathrm{O}_{2}$ and ATP evoke the $\mathrm{K}_{\text {ATP }}$ closure (and TRPM2 is independently activated by $\mathrm{H}_{2} \mathrm{O}_{2}$ ), or $\mathrm{K}_{\text {ATP }}$ closing is "classically" ensured by the elevated ATP, while $\mathrm{H}_{2} \mathrm{O}_{2}$ activates TRPM2 or other NSCCs provide the essential NSCC-mediated shift to the threshold depolarization. As a result, $\mathrm{Ca}_{V}$ channels are open, providing the $\mathrm{Ca}^{2+}$ signal for IGV exocytosis. The end products of the $\beta$-like oxidation of KIC, contributing to OXPHOS, are acetoacetate $(\mathrm{AcAc})$ and acetyl-CoA (AcCoA). AcCoA enters the Krebs cycle to form citrate. AcAc can be partly exported to the cytosol or is converted to $\beta$-hydroxybutyrate by a reverse reaction of $\beta$-hydroxybutyrate dehydrogenase at the expense of NADH. Moreover, since theoretically the amplifying mechanism should remain intact, we predict that also the net (experimental) ketoacidstimulated insulin secretion should be amplified by GLP1 and GIP (see Figure 2).

\subsection{Insulin Secretion Stimulated by Branched-Chain Ketoacids}

\subsubsection{Overview of Branched-Chain-Ketoacid-Stimulated Insulin Secretion}

One can envisage that the rules for GSIS are also valid for BCKA-stimulated insulin secretion. In both cases, the increased metabolism and Krebs cycle turnover resulting in elevated ATP synthesis is paralleled by the redox signaling. This stems from mitochondria for BCKA-stimulated insulin secretion or originates from the NOX4 reaction for GSIS. When both conditions are fulfilled, i.e., ATP plus $\mathrm{H}_{2} \mathrm{O}_{2}$ is elevated, this leads to conditions required for the $\mathrm{Ca}_{V}$ opening and IGV exocytosis (either ATP plus $\mathrm{H}_{2} \mathrm{O}_{2}$ are required for the $\mathrm{K}_{\text {ATP }}$ closure; or ATP closes $\mathrm{K}_{\text {ATP }}$ plus $\mathrm{H}_{2} \mathrm{O}_{2}$ activates some of NSCCs, such as TRPM2).

Additionally, the $\mathrm{Ca}^{2+}$ activation of $\mathrm{BCKDH}$ has an important self-accelerating role when the BCKA-stimulation of insulin secretion is initiated. The primary elevations of the 
cytosolic $\left[\mathrm{Ca}^{2+}\right]_{\mathrm{c}}$ due to the $\mathrm{Cav}$ opening are relayed to the increases in mitochondrial matrix $\left[\mathrm{Ca}^{2+}\right]_{\mathrm{m}}[99]$ and consequently activate BCKDH. So, in the next "turn", more intensive OXPHOS and redox signaling lead to more intensive insulin secretion.

\subsubsection{Physiological Context of Branched-Chain-Ketoacid-Stimulated Insulin Secretion}

BCAAs were previously found to stimulate insulin secretion in vivo in numerous reports, without a consensus on the mechanism. However, it was recognized that BCAAs can either amplify GSIS or act as secretagogues per se. The latter was confirmed to be based on the oxidative decarboxylation of the respective BCKAs [335]. Additionally, the ability of leucine to allosterically activate glutamate dehydrogenase was considered $[335,336]$.

The physiological context and relationships between the BCKA-stimulated insulin secretion and GSIS should be the subject of further studies. One can predict GSIS to be faster and BCKA-stimulated insulin secretion to be delayed from the onset of maximum insulin theoretically given by the net GSIS after a meal rich in saccharides and proteins. In contrast, the most typical situation for BCKA-stimulated insulin secretion can occur during fasting, when there are maximum levels of BCKAs in blood plasma.

\section{Mechanism of Fatty Acid-Stimulated Insulin Secretion \\ 6.1. Relevancy of Fatty Acid-Stimulated Insulin Secretion (FASIS) \\ 6.1.1. Experimental Approach vs. Physiology}

It is still controversial, as to whether there is fatty acid stimulated insulin secretion (FASIS) at low (insulin non-stimulating) glucose concentrations. Some previous observations suggested that glucose should always be present for FAs to induce any insulin secretion response [116,313,314,337]. However, several other observations described FASIS at low glucose concentrations (which alone do not stimulate insulin secretion) $[4,27,338,339]$, but being absent at zero glucose [339]. Nevertheless, human islets perfused at zero glucose do not increase their respiration upon the addition of long-chain FAs, but do release insulin [339]. Moreover, both respiration and insulin release were increased when long-chain FAs were added to human islets in the presence of $5.5 \mathrm{mM}$ glucose. These results fit well with FASIS having two components: a metabolic one and a component dependent on metabotropic receptor signaling [338].

Of course, one should consider the above-mentioned testing conditions with zero glucose as completely non-physiological. Since FAs trigger the action potential at a low, insulin non-stimulating glucose concentration in pancreatic $\beta$-cells [340], FASIS should be studied independently of GSIS. Physiologically, postprandial responses should exist due to all secretagogues resulting from major saccharide, fat and protein components. A mixed or fatty meal leads to the intestinal formation of chylomicrons. However, chylomicrons are brought by the circulation system to pancreatic islets with a delay, which amounts to a few hours in humans [341] and at least a half an hour to an hour in rodents [342,343]. Some authors reported that the lipid- or FA-mediated secretion of GLP-1 by intestinal L-enterocytes is instead instantaneous [344]. This contrasts with the findings of peaks in circulation for biologically active GLP-1 in human plasma 30-60 min after carbohydrate or protein intake and $120 \mathrm{~min}$ after the ingestion of lipids [274]. Hence it needs to be verified for humans whether the peak of GLP-1 coincides with the peak of chylomicrons in circulation, or whether GLP-1 precedes the peak of chylomicrons. The same is needed for rodents. This is important, because knowledge of precise timing can distinguish between GLP-1-amplified GSIS at intermediate glucose from the net FASIS. At least FAs mobilized from white adipose tissue stores should not coincide with the above phenomena, since lipolysis is inhibited by the secreted insulin [341].

Nevertheless, it seems plausible that a delayed insulin secretion due to chylomicrons should be expected when even the one-hour-lasting 2nd GSIS phase is nearly complete in rodents. At this time, the inhibition of lipolysis ceases due to the return of blood insulin levels and glycemia back to "fasting" values before the meal. However, the incoming chylomicrons should stimulate at least some response, and these responses would thus 
occur already at "fasting" glucose concentrations. The GLP-1 amplifying component (initiated due to intestinal lipids or FAs) may precede FASIS, especially if it still occurs during intermediate glycemia. Whether simultaneous responses to GLP-1 and FAs occur in vivo should also be studied. In conclusion, not only due to molecular mechanistic reasons, but due to the physiological timing, it is crucial to study FASIS experimentally. Of course, in supraphysiological experiments FASIS may overwhelm responses to the other secretagogues, especially when applied intraperitoneally or intravenously or when originating from an experimental high-fat diet [345].

\subsubsection{GPR119 Pathway in Pancreatic $\beta$-Cells}

Physiologically, one cannot separate FASIS from the concomitant portion of insulin secretion stimulated via 2-monoacylglycerols (MAGs). MAGs are agonists for another metabotropic receptor, GPR119, which provides signaling via G $\alpha$ s and cAMP $[184,263]$. Thus, all pathways relevant, e.g., for GLP1R are activated by GPR119. Additionally, the metabolic component of FASIS undoubtedly involves FA $\beta$-oxidation (see below), providing both ATP from the elevated OXPHOS and $\mathrm{H}_{2} \mathrm{O}_{2}$ from the enhanced superoxide formation by the respiratory chain and due to the ETFQOR input of $\mathrm{QH}_{2}$ to it, as with BCKAs. In conclusion, MAG-stimulated insulin secretion via GPR119 is also allowed due to the metabolic component, which can be provided by FAs under conditions of fasting glucose. Nevertheless, MAG-stimulation representing an amplification of GSIS is the end point of the so-called glycerol/FA cycle, described below.

\subsubsection{Physiological Stimulation of GPR40 and GPR119 Receptors in Pancreatic $\beta$-Cells}

Fatty acids appear in pancreatic islet capillaries either bound to albumin, being components of lipoproteins of endogenous sources, or part of postprandial chylomicrons resulting from dietary fat lipids. The latter are rich in triglycerides, which are cleaved locally in pancreatic islet capillaries by lipoprotein lipase [318]. The resulting MAGs and long-chain FAs [338,346-348] each stimulate their own receptor [270]. Adipose triglyceride lipase (ATGL) was reported to be the major isoform cleaving these triglycerides [349].

Secretory phospholipases A2 might also contribute to the free pool of long-chain FAs in the islet capillaries, in addition to PLC, residing on the plasma membrane and being inherent in GPR40 signaling [350]. In parallel, long-chain FAs are imported into pancreatic $\beta$-cells by the CD36 FA transporter. A fraction of CD36 molecules may be acetylated at several lysines, which blocks its function. Therefore, at elevated $\mathrm{NAD}^{+}$, which only occurs upon GSIS, sirtuins are activated and deacetylate the particular CD36 fraction [351]. As a result, the import of free long-chain FAs is promoted. Even this mechanism can be regarded as a certain type of potentiation of FASIS upon GSIS.

\subsubsection{Physiological Stimulation of Other Metabotropic Receptors in Pancreatic $\beta$-Cells}

Short-chain fatty acids act specifically via the GPR41 metabotropic receptor and contribute to a fine tuning of insulin secretion in both fed and fasting states [352,353]. Similarly, the metabotropic receptor GPR120, which has a different selectivity for agonists, mediates the amplification and/or stimulation of insulin secretion, by, e.g., $\alpha$-linolenic acid and polyunsaturated FAs [354].

\subsection{GPR40 and Metabolic Pathway upon Fatty Acid-Stimulated Insulin Secretion 6.2.1. GPR40 Pathway in Pancreatic $\beta$-Cells}

Theoretically, FASIS may contain one component that depends on the metabolism and a second component that relies on stimulation by the metabotropic receptor GPR40 [27,184,338,355-362]. Its activation is not involved in the FA chronic induction of lipotoxicity [363]. The FASIS mechanism that is dependent on GPR40 activation should be a major one, since the ablation of GPR40 or its point R258W mutation impaired FASIS [358]. 
The major pathway downstream of GPR40 acts via G $\alpha \mathrm{q} / 11$, activating PLC, releasing IP3 and DAG. Therefore, the most prominent pathway for FASIS under low glucose conditions should be GPR40-G $\alpha$ q/11-PLC-DAG-PKC, phosphorylating TRPM4 (TRPM5) channels and activating them, which would aid the necessary shift to the depolarization by the $100 \%$ closed $K_{\text {ATP }}$ ensemble. The $K_{\text {ATP }}$ closure should be ensured by the metabolic component of FASIS, which might, as illustrated in Figures 1 and 8, either cause closing of the entire $\mathrm{K}_{\mathrm{ATP}}$ ensemble; and simultaneously or alternatively to this, could activate TRPM2. The GPR40-G $\alpha$ /11-PLC-DAG pathway, signaling via PKC, reportedly leads to the phosphorylation and activation of another NSCC channel, TRPC3 [359]. The routes involving GPR40-G $\alpha$ q/11-PLC-IP3 activate the additional $\mathrm{Ca}^{2+}$ efflux from the ER initiated by the Cav opening [345].

The GPR40 receptor signaling also activates pathways of the signal-regulated kinase 1 and 2 (ERK1/2) [360]. Moreover, the downstream signaling of the GPR40 receptor slightly increases respiration [361]. GPR40 signaling was also implicated in the activation of p21activated kinase 4 (PAK4) [364]. Since PAK4 was known to be a regulator of cytoskeletal dynamics, this pathway may aid the facilitation of IGV exocytosis. Additionally, protein kinase D (PKD) was confirmed to be activated downstream of GPR40 and DAG [365].

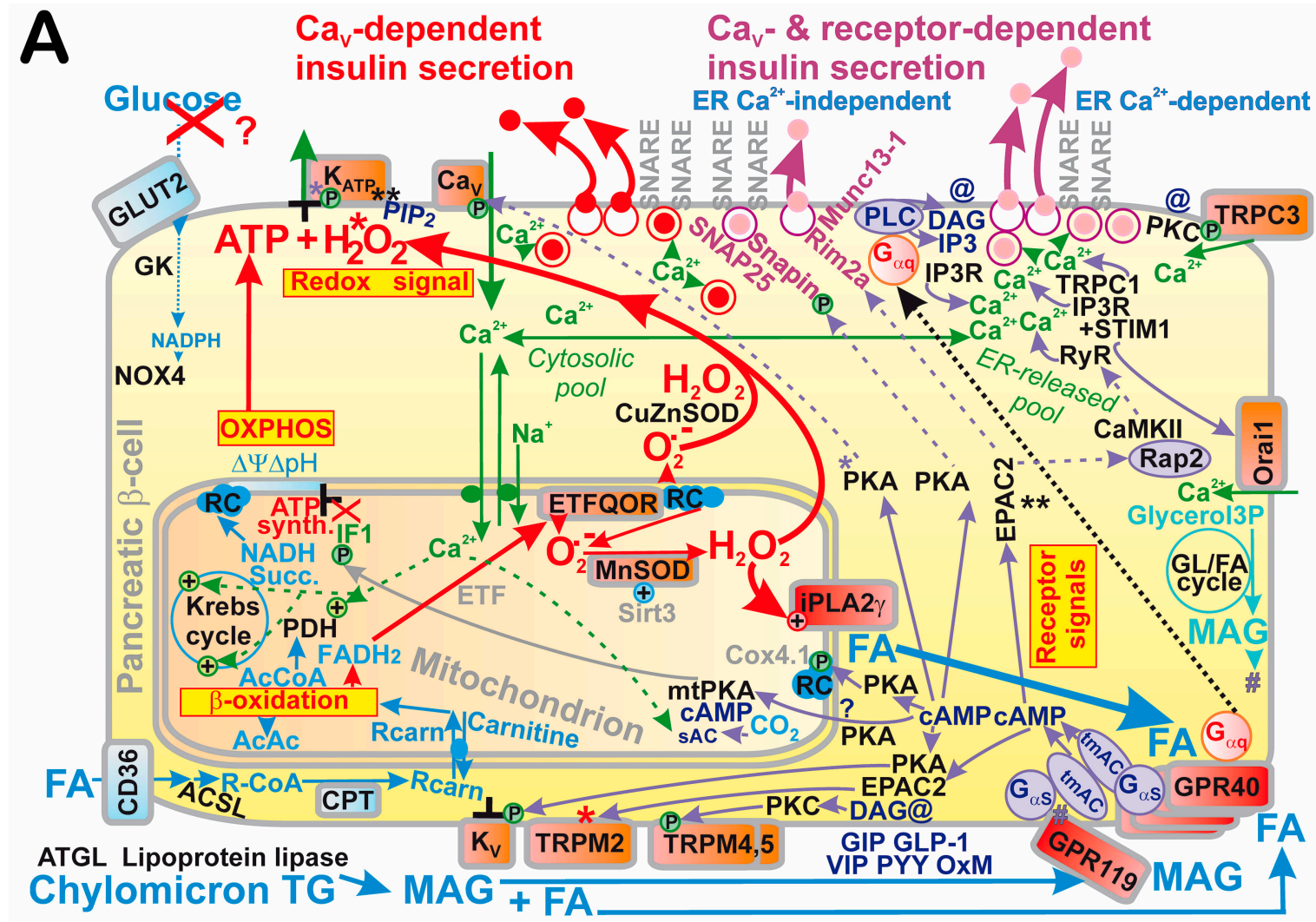

Figure 8. Cont. 


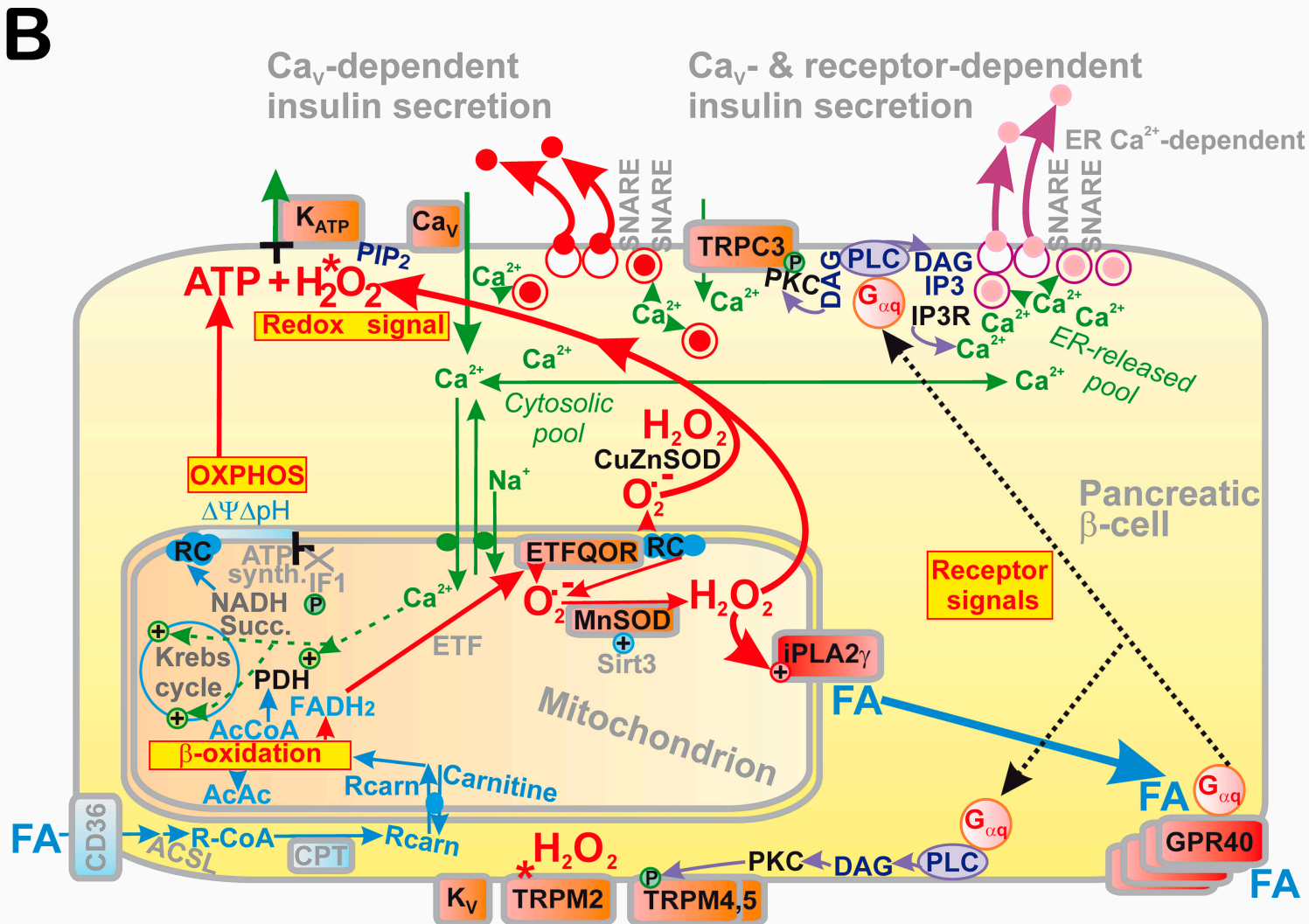

Figure 8. (A) Complex mechanism of FASIS and (B) GPR40 receptor part. FASIS is predominantly mediated by the GPR40 receptor. There are controversies over whether FAs bound to serum albumin and those cleaved by lipoprotein lipase can be transported into $\beta$-cells by FA transporter CD36 under fasting conditions. Such FA transport and cleavage of triglycerides (TG) to monoacylglycerols (MAG) and FA, however, take place when postprandial chylomicrons reach the rich capillaries of the pancreatic islets. MAG subsequently activates the metabotropic receptor GPR119, while long-chain FAs activate GPR40. The activations of both receptors lead to $\mathrm{Ca}_{\mathrm{V}}$-mediated action potential spikes and concomitant pulsatile insulin secretion. The canonical GPR119 signaling and biased GPR40 signaling leads to cAMP activation of the PKA and EPAC2 pathways with all the consequences described in the legends to Figures 2 and 7 . Our in vitro and in vivo experiments with mice (unpublished) demonstrated that approximately 2/3 of the GPR40 response is given by the amplifying mechanism due to the mitochondrial phospholipase iPLA2 $\gamma /$ PNPLA8, similarly as in INS-1E cells [309]. The phospholipase iPLA2 $\gamma$ is directly activated by the elevated $\mathrm{H}_{2} \mathrm{O}_{2}$ in the mitochondrial matrix and cleaves both saturated and unsaturated FAs from the phospholipids of mitochondrial membranes. The cleaved free FAs diffuse up to the plasma membrane, where they activate GPR40. FASIS in iPLA2 $\gamma$ knockout mice or its isolated islets yields $30 \%$ insulin in the 1st fast phase of insulin secretion, compared to wt mice (Holendová et al., unpublished). This supports the existence of such an acute mechanism in vivo. Besides the GPR40 pathway, a portion of FASIS still results from FA $\beta$-oxidation, with a similar mechanism as was described for ketoacids (see the Figure 7 legend). The difference lies in the several reaction turns during $\beta$-oxidation and a higher yield of $\mathrm{FADH}_{2} / \mathrm{QH}_{2}$ and AcCoA (and, of course ATP) from a single FA molecule. Theoretically, the stimulation of incretin receptors and PKA (and other kinase) pathways should also contribute to an amplification of responses to FAs. Nevertheless, the fraction of this contribution should be small due to a nearly overwhelming contribution of GPR40 and GPR119. Moreover, when FAs stimulate insulin secretion together with high glucose, the contribution of the so-called triglyceride/FA cycle, alternatively termed the glycerol-3-phosphate fatty acid cycle (G3P/FA cycle), also amplifies insulin secretion. This is provided by creating more ligands for the GPR40 and GPR119 receptor and by additional DAG signaling.

\subsection{2. $\beta$-Oxidation of Fatty Acids in Pancreatic $\beta$-Cells}

During GSIS, and because of the operation of the pyruvate/isocitrate and pyruvate/citrate shuttles, conditions are set to promote FA synthesis. Accordingly, metabolomics studies indicated a palmitic acid increase after the transition from low to high glucose conditions [307]. Note also that acyl-CoAs, as intermediates of FA $\beta$-oxidation, were 
suggested to stimulate/amplify insulin secretion [7]. In contrast, under low glucose conditions, FA $\beta$-oxidation readily proceeds in pancreatic $\beta$-cells, and therefore supplies the cell's needs when cells are depleted of glucose. Acyl-CoA synthetase converts FAs to acyl-CoAs, while the cytosolic carnitine acyltransferase 1 (CAT1) converts acyl-CoAs to acyl-carnitines [366]. Their uptake into the mitochondrial matrix is provided by the carnitine carrier (SLC25A20), which imports long-chain acyl carnitines in exchange for carnitine. Next, the matrix carnitine acyltransferase 2 (CAT 2) provides the conversion of acyl carnitines to acyl-CoAs. The following chain of reactions shortens the FA acyl chain by two carbons, involving acyl-CoA dehydrogenases, enoyl-CoA hydratase, 3-hydroxyacyl-CoA dehydrogenase and $\beta$-thiolase. The product of a single cycle is just acyl-CoA shortened by two carbons plus acetyl-CoA.

\subsection{3. $\beta$-Oxidation of Fatty Acids Produces Superoxide}

In general, FA $\beta$-oxidation involves mitochondrial flavoprotein dehydrogenases, such as the short-chain acyl-CoA dehydrogenase (EC 1.3.99.2); medium-chain acyl-CoA dehydrogenase (EC 1.3.99.3); long-chain acyl-CoA dehydrogenase (EC 1.3.99.13) and very longchain acyl-CoA dehydrogenase. They all donate electrons to ETFQOR via ETF, and therefore contribute to the mitochondrial superoxide formation, which after conversion to $\mathrm{H}_{2} \mathrm{O}_{2}$ serves as a redox signaling. As with the $\beta$-like oxidation of BCKAs, FA $\beta$-oxidation leads to elevated rates of mitochondrial superoxide formation, hence to redox signaling. The mechanism should be identical to that described above. Even under conditions of low glucose, the ETF/ ETFQOR redox relay to complex I of the mitochondrial respiratory chain plus the ETFQOR system serves as the electron acceptor for dehydrogenases of $\beta$-oxidation. Interestingly, GPR40 signaling also activates NOX2 [367].

\subsection{Redox-Sensitive Mitochondrial Phospholipase iPLA2 $\gamma$ Amplifies FASIS}

\subsubsection{FASIS at Low Glucose vs. Dependence of Metabolic and Receptor Part of FASIS}

There is the question of whether the GPR40-receptor-dependent component of FASIS also depends on the opening of $\mathrm{Ca}_{\mathrm{V}}$ channels. This is equivalent to another question: whether the GPR40-receptor component of FASIS is absolutely dependent on the metabolic (mitochondrial) component. Since there are action potential spikes due to $\mathrm{Ca}_{\mathrm{V}}$ opening upon GPR40 activation under low glucose conditions [340], we should conclude that there is at least experimental FASIS under low glucose conditions, and that the established metabolism under these conditions is sufficient to keep certain GPR40 downstream pathways operating and is thus able to stimulate insulin secretion. Speculatively, $\beta$ oxidation should provide conditions of elevated ATP plus $\mathrm{H}_{2} \mathrm{O}_{2}$ to trigger the "basic" portion of the IGV exocytosis, while the additional amplification provided by GPR40 should operate via the pathways described above in Section 6.2.1.

\subsubsection{Redox-Sensitive Mitochondrial Phospholipase iPLA2 $\gamma$ Amplifies FASIS}

Notably, the 1st phase of experimental FASIS was recognized (and the second only partly) to be amplified by the action of mitochondrial phospholipase iPLA2 $\gamma$, which provides the cleaved mitochondrial FAs to GPR40 in insulinoma INS-1E cells [309] and in mouse islets (Holendová et al., unpublished). iPLA2 $\gamma$ is directly activated by $\mathrm{H}_{2} \mathrm{O}_{2}$, hence it is also activated by the intramitochondrial redox signaling originating from FA $\beta$-oxidation. As a result, free long-chain FAs entering $\beta$-cells via CD36 and those cleaved from intracellular MAGs self-accelerate the GPR40 signaling and hence insulin secretion, after being imported into mitochondria and metabolized by $\beta$-oxidation. The activated iPLA2 $\gamma$ cleaves free long-chain FAs from mitochondrial phospholipids. The cleaved long-chain FAs diffuse to the plasma membrane and subsequently stimulate GPR40 (Figure 8). It should be investigated whether this is only allowed after their flip onto the outer phospholipid bilayer leaflet of the plasma membrane, i.e., onto their exterior. The plausibility of this event was suggested by an experiment in which activated iPLA2 $\gamma$ cleaved long-chain FAs, while their 
diffusion to the plasma membrane was indicated with the FA-sensitive fluorescent protein ADIFAB [309].

\subsection{FASIS at High Glucose}

\subsubsection{Distinction from FASIS at Low Glucose}

It is not surprising that in the presence of both FA and higher glucose concentrations (those stimulating GSIS when applied without FAs), the fraction of metabolic triggering increases. A more intensive intermittent $\mathrm{Ca}_{\mathrm{V}}$ opening with probably prolonged bursting may be a cause, which would induce an additional $\mathrm{Ca}^{2+}$ release from stores such as the ER, IGVs or lysosomes. At the same time, the GPR40-pathway stimulation of IGV exocytosis is likely to be maintained. These speculations are supported by so far the largest amount of insulin being secreted by INS-1E cells when palmitic acid plus $25 \mathrm{mM}$ glucose were added together, relative to either stimulation by palmitic acid alone or GSIS alone [338]. These aspects require further experimentation in vivo to more closely resembled physiological conditions.

\subsection{2. $\beta$-Oxidation vs. Triglyceride/Fatty Acid Cycle in Pancreatic $\beta$-Cells}

We discussed whether high glucose is essential for FASIS as such. Vice versa, a certain minimum level of FA metabolism was found to be required even for GSIS. This view was supported by experiments in which the inhibition of triglyceride lipolysis $[368,369]$ attenuated GSIS in isolated PIs and the 1st GSIS phase in mice with deleted lipase specifically in $\beta$-cells [370] or in ATGL knockout mice [349]. However, the net FASIS induced by palmitate was not affected by the ATGL deletion synthesis of triglycerides being alternated with triglyceride hydrolysis [20]. Such cycling, termed the triglyceride/fatty acid cycle is futile, since it consumes ATP. Nevertheless, one may speculate that since DAG is one of the intermediates of the cycle, it may provide all the benefits described above in previous sections. Moreover, free FAs released during the cycle may add supplemental FASIS to the net GSIS.

Indeed, the addition of FAs to $\beta$-cells and PIs in the presence of insulin-stimulating glucose amplifies GSIS, while $\beta$-cell acyl-CoA levels increase and appear to rapidly esterify glycerol 3-phosphate into lysophosphatidic acid and several different glycerolipids [308]. Note that this partly replenishes cytosolic $\mathrm{NAD}^{+}$. Additionally, glycerol-3-phosphatase produces glycerol and thus regulates glycolysis, the cellular redox state, ATP production and other important branches of metabolism [371]. The consequences of higher glycerol3-phosphate formation lie in the concomitant resulting increase in long-chain saturated MAGs [372,373]. These MAGs additionally stimulate insulin secretion via the GPR119 receptor and downstream PKA and EPAC2 pathways, the latter notably facilitates IGV priming by activating the protein Munc13-1 [372]. Note that this is similar to the GPR40 activation by the FAs cleaved in the cell interior, i.e., from the mitochondrial membranes by the mitochondrial phospholipase iPLA2 $\gamma$.

In INS-1E cells, about two-thirds of the 1st phase of FASIS was dependent on GPR40 and nearly the same $33 \%$ of the maximum FASIS amplitude was observed upon silencing iPLA2 $\gamma$ (or its ablation in mice, unpublished). The remaining FASIS can be considered to be dependent on the elevation of ATP plus redox signaling from $\beta$-oxidation.

Higher glucose also decreases acyl-CoA levels in pancreatic $\beta$-cells [62]. Previously, acyl-CoAs were suggested to activate $\mathrm{K}_{\mathrm{ATP}}$ [310], hence declining acyl-CoAs would ease $\mathrm{K}_{\mathrm{ATP}}$ closure. As we described in detail above, the metabolism of the remaining longchain acyl-CoAs leads to superoxide $/ \mathrm{H}_{2} \mathrm{O}_{2}$ formation, which aids the opening of $\mathrm{Ca}_{\mathrm{L}}$. In contrast, incoming higher glucose in pancreatic $\beta$-cells increases malonyl-CoA [62,374], which inhibits CPT1 and hence FA $\beta$-oxidation. This in turn opens the way for FA synthesis stemming from the ACL reaction after the citrate efflux from mitochondria [373]. Nevertheless, the silencing of ACL and fatty acid synthase in $\beta$-cells did not affect GSIS [311]. 


\subsection{GLP-1 as an Important Stimulus for Postprandial Insulin Secretion after High Fat Meal}

One can hypothesize that during fasting the increased FAs bound to albumin in circulation contribute to the ongoing basal secretion of insulin upon fasting [27]. However, it is necessary to investigate whether these minimum, non-pathological levels of FAs bound to plasma albumin possess the ability to stimulate a "basal" FASIS. Nevertheless, the ability to activate GPR40 by the FAs-bound to albumin was questioned [375,376]. Hypothetically, non-pathological levels of FAs in circulation upon fasting may stimulate GLP-1 secretion in intestinal enterocyte L-cells. The resulting basal GLP-1 can therefore stimulate a certain level of insulin secretion in pancreatic $\beta$-cells. This speculation will be plausible once there is definitive evidence that GLP-1 stimulates insulin secretion under low glucose conditions.

In contrast, upon high glucose intake, until chylomicrons arrive, albumin-bound FA levels are minimum, because the lipolysis is simultaneously inhibited in white adipose tissue due to the blockage of adipocyte lipases upon ongoing insulin receptor signaling, and so glucose uptake to adipocytes predominates [377]. The timing of the occurrence of GLP-1 in human circulation exhibits a peak of $<10 \mathrm{pmol} / \mathrm{L}$ [267] between 30 and 60 min after carbohydrate or protein intake, and $120 \mathrm{~min}$ after the ingestion of lipids [274]. When compared to the availability of chylomicrons (see above), responses to GLP-1 should precede those of chylomicrons. However, their overlap is not excluded.

\subsection{Other Sectretagogues}

The arginine-mediated amplification of insulin secretion was previously reported to be independent of nitric oxide synthase [378]. Arginine effects related to insulin secretion are also mediated by the metabotropic receptor GPR6A [379]. Additionally, adenylosuccinate (S-AMP) was found to rise in response to glucose, and may be considered to be a secretagogue [380]. The Conversion of IMP to S-AMP is provided by the adenylosuccinate synthase. S-AMP then activates the sentrin/SUMO-specific protease SENP1 [381], which influences redox homeostasis similarly to the glutathione/glutaredoxin system [13].

\section{Mechanisms of Transfer of Redox Signaling and Possible Insults}

\subsection{Mechanims of Transfer of Redox Signaling}

Mechanisms of redox signal conduction within the cell can either rely on a simple $\mathrm{H}_{2} \mathrm{O}_{2}$ diffusion or on the redox relay mediated by thiol-based proteins that reversibly form S-S bridges and are capable of transferring such a transient oxidation to the target [14]. Peroxiredoxins are such proteins, and their oxidation states are regenerated via thioredoxins and glutaredoxins. Redox signal transfer over a distance, which can be as far as several $100 \mathrm{~nm}$, would significantly decay when conducted by simple $\mathrm{H}_{2} \mathrm{O}_{2}$ diffusion, due to nonspecific reactions with random targets within the cytosolic (membrane) milieu. In contrast, the peroxiredoxin oxidized into the first stage can diffuse and transfer the redox signal by oxidation of the distal protein target [14]. It could be speculated that a mutually interacting array of peroxiredoxin conducts the redox signal instead of their diffusion.

Additionally, the so-called floodgate model could explain the spreading of a redox signal over a larger distance [1,15]. The model is based upon the ability of peroxiredoxin to be oxidized into sulfenyls, sulfinil and sulfoxyl stages. Moreover, these two highly oxidized stages form clusters of regular decamers. A local $\mathrm{H}_{2} \mathrm{O}_{2}$ source then oxidizes peroxiredoxin decamers, which do not escape from its proximity, into their high oxidized states. As a result, the only ones that escape by diffusion are kept in the lowest oxidized stages and can oxidize the proper specific target in a sufficient distance. Experimentally, targets such as a signal transducer and activator of transcription 3 (STAT3) or apoptosis signal-regulating kinase 1 (ASK1) were confirmed to react with peroxiredoxin 2 and 1 , respectively, in this way [382-385]. Targets relevant for GSIS and their interactions with peroxiredoxin and/or $\mathrm{H}_{2} \mathrm{O}_{2}$ are yet to be determined, as discussed in Sections 2.3.1 and 2.3.2.

Note, however, that for high levels of $\mathrm{H}_{2} \mathrm{O}_{2}$, its detoxification is ensured by the peroxiredoxin/thioredoxin antioxidant system $[386,387]$. As was already mentioned in the Introduction, the glutathione content is relatively low in pancreatic $\beta$-cells [8-11]. Since in 
contrast, the relative content of thioredoxins and glutaredoxins [10,11], and peroxiredoxins and other proteins capable of redox relay is satisfactorily high, the above-mentioned redoxrelay spread of redox signals is easily conducted in such a low-glutathione milieu [14,15]. In conclusion, the pancreatic $\beta$-cell employs a plethora of redox signals for the acute stimulation of insulin secretion. The appearance of those signals (or their frequency) may hypothetically maintain the correct transcriptome to maintain $\beta$-cell identity $[4,17]$.

\subsection{Redox Status of Pancreatic $\beta$-Cells may Affect and Impact Redox Signaling Essential for Insulin Secretion}

Dor and colleagues hypothesized that repeating $\mathrm{K}_{\mathrm{ATP}}$-dependent insulin exocytosis and all ionic and regulatory phenomena involved also contribute to the maintenance of the insulin gene expression, hence to the maintenance of so-called $\beta$-cell identity [17]. Subsequently, redox signaling, which is essential for GSIS and also for the stimulation of insulin secretion by other secretagogues (BCKAs and FAs), is also expected to be one of the key factors to maintain $\beta$-cell identity [16]. These aspects require further research. Incorrect checking of $\beta$-cell identity (meaning dysregulation of proper signaling maintaining proper expression of $\beta$-cell-specific genes and suppression of so-called "disallowed" genes) may subsequently lead to particular stress signals to the periphery, such as those initiating development of peripheral insulin resistance. This progresses to type- 2 diabetes development. Indeed, we found that a single gene ablation, ablation of NOX4 responsible for the majority of redox signaling upon GSIS, generates the onset of insulin resistance [4]. We could speculate that in this case, pancreatic $\beta$-cells must emit an as of yet unidentified stress signal (directly or via the immune system to the periphery) just because of the insufficient identity checking or autocrine self-maintenance of $\beta$-cells in mice with ablated NOX4.

It has long been assumed that pancreatic $\beta$-cells are particularly vulnerable to oxidative stress because of the relatively low expression of several antioxidant enzymes, including catalase, superoxide dismutase and GSH peroxidase [8,388]. However, recent findings identify the peroxiredoxin/thioredoxin antioxidant system as the major detoxifying pathway of $\beta$-cells $[386,387]$. Thus, it has been shown that $\beta$-cells have the capacity to detoxify micromolar levels of $\mathrm{H}_{2} \mathrm{O}_{2}$ through an endogenous thioredoxin reductase-dependent mechanism, a finding that can be masked by the use of nonphysiological bolus delivery of oxidants to the experimental system [386]. Given the catalytic efficiencies of peroxiredoxins for $\mathrm{H}_{2} \mathrm{O}_{2}\left(10^{5} \mathrm{M}^{-1} \mathrm{~s}^{-1}\right)$ [389], the affinity of peroxiredoxins for $\mathrm{H}_{2} \mathrm{O}_{2}\left(\mathrm{~K}_{\mathrm{m}}<20 \mu \mathrm{M}\right)$ and the property of peroxiredoxins to be deactivated through oxidation, it has been suggested that peroxiredoxins are the prime candidates for mediators of $\mathrm{H}_{2} \mathrm{O}_{2}$ signaling in addition to detoxification in $\beta$-cells $[14,386,387]$. Based on these suggestions, we hypothesized that the NOX4-produced and mitochondria-generated $\mathrm{H}_{2} \mathrm{O}_{2}$ at GSIS in vivo could also be maintained in balance by the peroxiredoxin/thioredoxin antioxidant system. Oxidative stress that contributes to diabetic etiology occurs when such redox homeostasis is impaired.

Additionally excessive antioxidant protection may harm basic processes in pancreatic $\beta$-cells [4]. For example, cytosol-targeted antioxidant therapy would inevitably suppress GSIS due to (partial) elimination of redox signaling. Such therapy should be inappropriate even for the early stages of diabetes. Tuning down the essential $\mathrm{H}_{2} \mathrm{O}_{2}$ release at GSIS would instead amplify prediabetes symptoms instead of preventing them. In contrast, we predicted that mitochondria-targeted antioxidants should not harm the physiological redox signaling (except that of BCKAs and FAs) and may be better tolerated when the premature oxidative stress in the matrix at the prediabetic stage is treated in this way [4].

The term mitochondrial hormesis, or mitohormesis, has been introduced to describe a defense mechanism observed in oxidant-induced stress-responses by mitochondria [390]. Persistent decrease of mitochondrial oxidative phosphorylation and the activity of respiratory complexes are associated with the release of oxidants from nonmitochondrial sources, together with the release of proinflammatory and profibrotic cytokines, and a manifestation of organ dysfunction. Consequently, restoration of mitochondrial function and superoxide 
production via activation of AMPK has been associated with improvement in markers of renal, cardiovascular and neuronal dysfunction with diabetes [391].

In addition, putative redox-active compounds, such as dietary antioxidants and olive oil nutraceuticals, are being studied as potential regulators of $\beta$-cell redox homeostasis, insulin secretion, blood glucose homeostasis and the development of type 2 diabetes (for selected recent reviews, see [392-396]). Considering the mechanism of action of dietary phytochemicals, we would like to alleviate the general belief that these compounds act as direct free radical scavengers in vivo. Kinetic constraints, given by the peroxiredoxin/thioredoxin antioxidant system in $\beta$-cells, indicate that in vivo one-electron scavenging of radicals by dietary antioxidants is ineffective in antioxidant defense. Enzymatic removal of non-radical electrophiles, such as hydroperoxides, in two-electron redox reactions is the primary antioxidant mechanism [397]. It has been proposed that a major mechanism of action for nutritional antioxidants is the paradoxical oxidative activation of the NRF2 (NF-E2-related factor 2) signaling pathway, which maintains protective oxidoreductases and their nucleophilic substrates [397-399]. The nutritional compounds are thus redox-active by activating signaling pathways, such as NRF2, and mimic the effect of endogenously produced electrophiles, a mechanism termed parahormesis [398]. Nevertheless, the detailed description of the role of nutrition, dietary polyphenols and their relation to the etiology of type 2 diabetes is beyond the scope of this review. A reader can refer to our previous review and to other relevant reviews published on the topic [16,396,400-402].

Author Contributions: Conceptualization, P.J.; resources B.H.; writing—original draft preparation, P.J.; writing-review and editing, P.J., B.H., A.D., M.J., J.T., L.P.-H.; funding acquisition, P.J. All authors have read and agreed to the published version of the manuscript.

Funding: This research was funded by the Grant Agency of the Czech Republic (Grantová Agentura České Republiky, GAČR), grant number 20-00408S.

Acknowledgments: The authors gratefully acknowledge the precise frequent renumbering of references during the manuscript preparation by B.H.

Conflicts of Interest: The authors declare no conflict of interest.

\section{References}

1. Reczek, C.R.; Chandel, N.S. ROS-dependent signal transduction. Curr. Opin. Cell Biol. 2015, 33, 8-13. [CrossRef] [PubMed]

2. Bell, E.L.; Klimova, T.A.; Eisenbart, J.; Moraes, C.T.; Murphy, M.P.; Budinger, G.R.; Chandel, N.S. The Qo site of the mitochondrial complex III is required for the transduction of hypoxic signaling via reactive oxygen species production. J. Cell Biol. 2007, 177, 1029-1036. [CrossRef] [PubMed]

3. Briggs, K.J.; Koivunen, P.; Cao, S.; Backus, K.M.; Olenchock, B.A.; Patel, H.; Zhang, Q.; Signoretti, S.; Gerfen, G.J.; Richardson, A.L.; et al. Paracrine Induction of HIF by Glutamate in Breast Cancer: EglN1 Senses Cysteine. Cell 2016, 166, 126-139. [CrossRef] [PubMed]

4. Plecita-Hlavata, L.; Jaburek, M.; Holendova, B.; Tauber, J.; Pavluch, V.; Berkova, Z.; Cahova, M.; Schroeder, K.; Brandes, R.P.; Siemen, D.; et al. Glucose-Stimulated Insulin Secretion Fundamentally Requires H2O2 Signaling by NADPH Oxidase 4 . Diabetes 2020. [CrossRef]

5. Sakaguchi, R.; Mori, Y. Transient receptor potential (TRP) channels: Biosensors for redox environmental stimuli and cellular status. Free Radic. Biol. Med. 2020, 146, 36-44. [CrossRef]

6. Kakei, M.; Yoshida, M.; Dezaki, K.; Ito, K.; Yamada, H.; Funazaki, S.; Kawakami, M.; Sugawara, H.; Yada, T. Glucose and GTP-binding protein-coupled receptor cooperatively regulate transient receptor potential-channels to stimulate insulin secretion [Review]. Endocr. J. 2016, 63, 867-876. [CrossRef]

7. Prentki, M.; Joly, E.; El-Assaad, W.; Roduit, R. Malonyl-CoA signaling, lipid partitioning, and glucolipotoxicity: Role in beta-cell adaptation and failure in the etiology of diabetes. Diabetes 2002, 51 (Suppl. 3), S405-S413. [CrossRef]

8. Lenzen, S. Oxidative stress: The vulnerable beta-cell. Biochem. Soc. Trans. 2008, 36, 343-347. [CrossRef]

9. Lenzen, S. Chemistry and biology of reactive species with special reference to the antioxidative defence status in pancreatic ß-cells. Biochim. Et Biophys. Acta. Gen. Subj. 2017, 1861, 1929-1942. [CrossRef]

10. Lenzen, S.; Drinkgern, J.; Tiedge, M. Low antioxidant enzyme gene expression in pancreatic islets compared with various other mouse tissues. Free Radic. Biol. Med. 1996, 20, 463-466. [CrossRef] 
11. Welsh, N.; Margulis, B.; Borg, L.A.; Wiklund, H.J.; Saldeen, J.; Flodström, M.; Mello, M.A.; Andersson, A.; Pipeleers, D.G.; Hellerström, C. Differences in the expression of heat-shock proteins and antioxidant enzymes between human and rodent pancreatic islets: Implications for the pathogenesis of insulin-dependent diabetes mellitus. Mol. Med. (Camb. Mass.) 1995, 1, 806-820. [CrossRef]

12. Ivarsson, R.; Quintens, R.; Dejonghe, S.; Tsukamoto, K.; Veld, P.; Renström, E.; Schuit, F.C. Redox control of exocytosis: Regulatory role of NADPH, thioredoxin, and glutaredoxin. Diabetes 2005, 54, 2132-2142. [CrossRef] [PubMed]

13. Reinbothe, T.M.; Ivarsson, R.; Li, D.-Q.; Niazi, O.; Jing, X.; Zhang, E.; Stenson, L.; Bryborn, U.; Renström, E. Glutaredoxin-1 Mediates NADPH-Dependent Stimulation of Calcium-Dependent Insulin Secretion. Mol. Endocrinol. 2009, 23, 893-900. [CrossRef] [PubMed]

14. Jezek, P.; Holendova, B.; Plecita-Hlavata, L. Redox Signaling from Mitochondria: Signal Propagation and Its Targets. Biomolecules 2020, 10, 93. [CrossRef]

15. Woo, H.A.; Yim, S.H.; Shin, D.H.; Kang, D.; Yu, D.Y.; Rhee, S.G. Inactivation of peroxiredoxin I by phosphorylation allows localized $\mathrm{H}(2) \mathrm{O}(2)$ accumulation for cell signaling. Cell 2010, 140, 517-528. [CrossRef] [PubMed]

16. Ježek, P.; Jabůrek, M.; Plecitá-Hlavatá, L. Contribution of Oxidative Stress and Impaired Biogenesis of Pancreatic $\beta$-Cells to Type 2 Diabetes. Antioxid. Redox Signal. 2019. [CrossRef]

17. Swisa, A.; Glaser, B.; Dor, Y. Metabolic Stress and Compromised Identity of Pancreatic Beta Cells. Front. Genet. 2017, 8, 21. [CrossRef]

18. Ashcroft, F.M.; Rorsman, P. Diabetes Mellitus and the $\beta$ Cell: The Last Ten Years. Cell 2012, 148, 1160-1171. [CrossRef]

19. Maechler, P. Mitochondrial function and insulin secretion. Mol. Cell. Endocrinol. 2013. [CrossRef]

20. Prentki, M.; Matschinsky, F.M.; Madiraju, S.R.M. Metabolic Signaling in Fuel-Induced Insulin Secretion. Cell Metab. 2013, 18, 162-185. [CrossRef]

21. Rutter, G.A.; Pullen, T.J.; Hodson, D.J.; Martinez-Sanchez, A. Pancreatic $\beta$-cell identity, glucose sensing and the control of insulin secretion. Biochem. J. 2015, 466, 203-218. [CrossRef] [PubMed]

22. Straub, S.G.; Sharp, G.W. Glucose-stimulated signaling pathways in biphasic insulin secretion. Diabetes/Metab. Res. Rev. 2002, 18, 451-463. [CrossRef] [PubMed]

23. Henquin, J.C. Regulation of insulin secretion: A matter of phase control and amplitude modulation. Diabetologia 2009, 52, 739-751. [CrossRef] [PubMed]

24. Seino, S.; Sugawara, K.; Yokoi, N.; Takahashi, H. $\beta$-Cell signalling and insulin secretagogues: A path for improved diabetes therapy. DiabetesObes. Metab. 2017, 19 (Suppl. 1), 22-29. [CrossRef] [PubMed]

25. Shibasaki, T.; Takahashi, T.; Takahashi, H.; Seino, S. Cooperation between cAMP signalling and sulfonylurea in insulin secretion. DiabetesObes. Metab. 2014, 16 (Suppl. 1), 118-125. [CrossRef] [PubMed]

26. Seino, S. Cell signalling in insulin secretion: The molecular targets of ATP, cAMP and sulfonylurea. Diabetologia 2012, 55, 2096-2108. [CrossRef]

27. Ježek, P.; Jabůrek, M.; Holendová, B.; Plecitá-Hlavatá, L. Fatty Acid-Stimulated Insulin Secretion vs. Lipotoxicity. Molecules 2018, 23, 1483. [CrossRef]

28. Leloup, C.; Tourrel-Cuzin, C.; Magnan, C.; Karaca, M.; Castel, J.; Carneiro, L.; Colombani, A.-L.; Ktorza, A.; Casteilla, L.; Penicaud, L. Mitochondrial Reactive Oxygen Species Are Obligatory Signals for Glucose-Induced Insulin Secretion. Diabetes 2009, 58, 673-681. [CrossRef]

29. Saadeh, M.; Ferrante, T.C.; Kane, A.; Shirihai, O.; Corkey, B.E.; Deeney, J.T. Reactive Oxygen Species Stimulate Insulin Secretion in Rat Pancreatic Islets: Studies Using Mono-Oleoyl-Glycerol. PLoS ONE 2012, 7, e30200. [CrossRef]

30. Rebelato, E.; Abdulkader, F.; Curi, R.; Carpinelli, A.R. Control of the Intracellular Redox State by Glucose Participates in the Insulin Secretion Mechanism. PLoS ONE 2011, 6, e24507. [CrossRef]

31. Pi, J.; Bai, Y.; Zhang, Q.; Wong, V.; Floering, L.M.; Daniel, K.; Reece, J.M.; Deeney, J.T.; Andersen, M.E.; Corkey, B.E.; et al. Reactive Oxygen Species as a Signal in Glucose-Stimulated Insulin Secretion. Diabetes 2007, 56, 1783-1791. [CrossRef] [PubMed]

32. Morgan, D.; Rebelato, E.; Abdulkader, F.; Graciano, M.F.R.; Oliveira-Emilio, H.R.; Hirata, A.E.; Rocha, M.S.; Bordin, S.; Curi, R.; Carpinelli, A.R. Association of NAD(P)H Oxidase with Glucose-Induced Insulin Secretion by Pancreatic $\beta$-Cells. Endocrinology 2009, 150, 2197-2201. [CrossRef] [PubMed]

33. Imoto, H.; Sasaki, N.; Iwase, M.; Nakamura, U.; Oku, M.; Sonoki, K.; Uchizono, Y.; Iida, M. Impaired Insulin Secretion by Diphenyleneiodium Associated with Perturbation of Cytosolic $\mathrm{Ca}^{2+}$ Dynamics in Pancreatic $\beta$-Cells. Endocrinology 2008, 149, 5391-5400. [CrossRef]

34. Syed, I.; Kyathanahalli, C.N.; Kowluru, A. Phagocyte-like NADPH oxidase generates ROS in INS 832/13 cells and rat islets: Role of protein prenylation. Am. J. Physiol. Regul. Integr. Comp. Physiol. 2011, 300, R756-R762. [CrossRef] [PubMed]

35. Li, N.; Li, B.; Brun, T.; Deffert-Delbouille, C.; Mahiout, Z.; Daali, Y.; Ma, X.-J.; Krause, K.-H.; Maechler, P. NADPH Oxidase NOX2 Defines a New Antagonistic Role for Reactive Oxygen Species and cAMP/PKA in the Regulation of Insulin Secretion. Diabetes 2012, 61, 2842-2850. [CrossRef]

36. Bouzakri, K.; Veyrat-Durebex, C.; Holterman, C.; Arous, C.; Barbieux, C.; Bosco, D.; Altirriba, J.; Alibashe, M.; Tournier, B.B.; Gunton, J.E.; et al. Beta-Cell-Specific Expression of Nicotinamide Adenine Dinucleotide Phosphate Oxidase 5 Aggravates High-Fat Diet-Induced Impairment of Islet Insulin Secretion in Mice. Antioxid. Redox Signal. 2020, 32, 618-635. [CrossRef] 
37. Plecitá-Hlavatá, L.; Engstová, H.; Holendová, B.; Tauber, J.; Špaček, T.; Petrásková, L.; Křen, V.; Špačková, J.; Gotvaldová, K.; Ježek, J.; et al. Mitochondrial Superoxide Production Decreases on Glucose-Stimulated Insulin Secretion in Pancreatic $\beta$ Cells Due to Decreasing Mitochondrial Matrix NADH/NAD(+) Ratio. Antioxid. Redox Signal. 2020. [CrossRef]

38. Spégel, P.; Sharoyko, V.V.; Goehring, I.; Danielsson, A.P.; Malmgren, S.; Nagorny, C.L.; Andersson, L.E.; Koeck, T.; Sharp, G.W.; Straub, S.G.; et al. Time-resolved metabolomics analysis of $\beta$-cells implicates the pentose phosphate pathway in the control of insulin release. Biochem. J. 2013, 450, 595-605. [CrossRef]

39. Bedard, K.; Krause, K.-H. The NOX Family of ROS-Generating NADPH Oxidases: Physiology and Pathophysiology. Physiol. Rev. 2007, 87, 245-313. [CrossRef]

40. Serrander, L.; Cartier, L.; Bedard, K.; Banfi, B.; Lardy, B.; Plastre, O.; Sienkiewicz, A.; Fórró, L.; Schlegel, W.; Krause, K.-H. NOX4 activity is determined by mRNA levels and reveals a unique pattern of ROS generation. Biochem. J. 2007, 406, 105-114. [CrossRef]

41. Di Fulvio, M.; Aguilar-Bryan, L. Chloride transporters and channels in $\beta$-cell physiology: Revisiting a 40-year-old model. Biochem. Soc. Trans. 2019, 47, 1843-1855. [CrossRef] [PubMed]

42. Rao, A.; McBride, E.L.; Zhang, G.; Xu, H.; Cai, T.; Notkins, A.L.; Aronova, M.A.; Leapman, R.D. Determination of secretory granule maturation times in pancreatic islet $\beta$-cells by serial block face scanning electron microscopy. J. Struct. Biol. 2020. [CrossRef]

43. Ma, W.; Chang, J.; Tong, J.; Ho, U.; Yau, B.; Kebede, M.A.; Thorn, P. Arp2/3 nucleates F-actin coating of fusing insulin granules in pancreatic $\beta$ cells to control insulin secretion. J. Cell Sci. 2020, 133. [CrossRef] [PubMed]

44. Meda, P.; Bosco, D.; Chanson, M.; Giordano, E.; Vallar, L.; Wollheim, C.; Orci, L. Rapid and reversible secretion changes during uncoupling of rat insulin-producing cells. J. Clin. Investig. 1990, 86, 759-768. [CrossRef]

45. Meda, P. The role of gap junction membrane channels in secretion and hormonal action. J. Bioenerg. Biomembr. 1996, 28, 369-377. [CrossRef]

46. Ravier, M.A.; Güldenagel, M.; Charollais, A.; Gjinovci, A.; Caille, D.; Söhl, G.; Wollheim, C.B.; Willecke, K.; Henquin, J.C.; Meda, P. Loss of connexin 36 channels alters beta-cell coupling, islet synchronization of glucose-induced Ca2+ and insulin oscillations, and basal insulin release. Diabetes 2005, 54, 1798-1807. [CrossRef]

47. Jacob, S.; Köhler, M.; Tröster, P.; Visa, M.; García-Prieto, C.F.; Alanentalo, T.; Moede, T.; Leibiger, B.; Leibiger, I.B.; Berggren, P.O. In vivo $\mathrm{Ca}(2+)$ dynamics in single pancreatic $\beta$ cells. Faseb J. Off. Publ. Fed. Am. Soc. Exp. Biol. 2020, 34, 945-959. [CrossRef]

48. Johnston, N.R.; Mitchell, R.K.; Haythorne, E.; Pessoa, M.P.; Semplici, F.; Ferrer, J.; Piemonti, L.; Marchetti, P.; Bugliani, M.; Bosco, D.; et al. Beta Cell Hubs Dictate Pancreatic Islet Responses to Glucose. Cell Metab. 2016, 24, 389-401. [CrossRef]

49. Rutter, G.A.; Hodson, D.J.; Chabosseau, P.; Haythorne, E.; Pullen, T.J.; Leclerc, I. Local and regional control of calcium dynamics in the pancreatic islet. DiabetesObes. Metab. 2017, 19 (Suppl. 1), 30-41. [CrossRef]

50. Leturque, A.; Brot-Laroche, E.; Le Gall, M. GLUT2 mutations, translocation, and receptor function in diet sugar managing. Am. J. Physiol. Endocrinol. Metab. 2009, 296, E985-E992. [CrossRef]

51. Kaminski, M.T.; Lenzen, S.; Baltrusch, S. Real-time analysis of intracellular glucose and calcium in pancreatic beta cells by fluorescence microscopy. Biochim. Et Biophys. Acta 2012, 1823, 1697-1707. [CrossRef] [PubMed]

52. Park, J.H.; Kim, S.J.; Park, S.H.; Son, D.G.; Bae, J.H.; Kim, H.K.; Han, J.; Song, D.K. Glucagon-like peptide-1 enhances glucokinase activity in pancreatic beta-cells through the association of Epac2 with Rim2 and Rab3A. Endocrinology 2012, 153, 574-582. [CrossRef] [PubMed]

53. Matschinsky, F.M.; Wilson, D.F. The Central Role of Glucokinase in Glucose Homeostasis: A Perspective 50 Years After Demonstrating the Presence of the Enzyme in Islets of Langerhans. Front. Physiol. 2019, 10, 148. [CrossRef] [PubMed]

54. Schuit, F.; De Vos, A.; Farfari, S.; Moens, K.; Pipeleers, D.; Brun, T.; Prentki, M. Metabolic fate of glucose in purified islet cells. Glucose-regulated anaplerosis in beta cells. J. Biol. Chem. 1997, 272, 18572-18579. [CrossRef] [PubMed]

55. Zhang, Z.; Liew, C.W.; Handy, D.E.; Zhang, Y.; Leopold, J.A.; Hu, J.; Guo, L.; Kulkarni, R.N.; Loscalzo, J.; Stanton, R.C. High glucose inhibits glucose-6-phosphate dehydrogenase, leading to increased oxidative stress and beta-cell apoptosis. Faseb J. Off. Publ. Fed. Am. Soc. Exp. Biol. 2010, 24, 1497-1505. [CrossRef]

56. Huang, M.; Joseph, J.W. Metabolomic analysis of pancreatic $\beta$-cell insulin release in response to glucose. Islets 2012, 4, $210-222$. [CrossRef]

57. Goehring, I.; Sauter, N.S.; Catchpole, G.; Assmann, A.; Shu, L.; Zien, K.S.; Moehlig, M.; Pfeiffer, A.F.H.; Oberholzer, J.; Willmitzer, L.; et al. Identification of an intracellular metabolic signature impairing beta cell function in the rat beta cell line INS-1E and human islets. Diabetologia 2011, 54, 2584-2594. [CrossRef]

58. Ammon, H.P.; Steinke, J. 6-Amnionicotinamide (6-AN) as a diabetogenic agent. In vitro and in vivo studies in the rat. Diabetes 1972, 21, 143-148. [CrossRef]

59. Verspohl, E.J.; Händel, M.; Ammon, H.P. Pentosephosphate shunt activity of rat pancreatic islets: Its dependence on glucose concentration. Endocrinology 1979, 105, 1269-1274. [CrossRef]

60. Monte Alegre, S.; Saad, S.T.; Delatre, E.; Saad, M.J. Insulin secretion in patients deficient in glucose-6-phosphate dehydrogenase. Horm. Metab. Res. Horm. Und Stoffwechs. Horm. Et Metab. 1991, 23, 171-173. [CrossRef]

61. Akhmedov, D.; De Marchi, U.; Wollheim, C.B.; Wiederkehr, A. Pyruvate dehydrogenase E1 $\alpha$ phosphorylation is induced by glucose but does not control metabolism-secretion coupling in INS-1E clonal $\beta$-cells. Biochim. Et Biophys. Acta 2012, 1823, 1815-1824. [CrossRef] [PubMed] 
62. Lorenz, M.A.; El Azzouny, M.A.; Kennedy, R.T.; Burant, C.F. Metabolome response to glucose in the $\beta$-cell line INS-1 832/13. J. Biol. Chem. 2013, 288, 10923-10935. [CrossRef] [PubMed]

63. Alves, T.C.; Pongratz, R.L.; Zhao, X.; Yarborough, O.; Sereda, S.; Shirihai, O.; Cline, G.W.; Mason, G.; Kibbey, R.G. Integrated, Step-Wise, Mass-Isotopomeric Flux Analysis of the TCA Cycle. Cell Metab. 2015, 22, 936-947. [CrossRef]

64. Ouyang, Q.; Nakayama, T.; Baytas, O.; Davidson, S.M.; Yang, C.; Schmidt, M.; Lizarraga, S.B.; Mishra, S.; Ei-Quessny, M.; Niaz, S.; et al. Mutations in mitochondrial enzyme GPT2 cause metabolic dysfunction and neurological disease with developmental and progressive features. Proc. Natl. Acad. Sci. USA 2016, 113, E5598-E5607. [CrossRef] [PubMed]

65. Yang, R.Z.; Park, S.; Reagan, W.J.; Goldstein, R.; Zhong, S.; Lawton, M.; Rajamohan, F.; Qian, K.; Liu, L.; Gong, D.W. Alanine aminotransferase isoenzymes: Molecular cloning and quantitative analysis of tissue expression in rats and serum elevation in liver toxicity. Hepatol. (Baltim. Md.) 2009, 49, 598-607. [CrossRef]

66. Maechler, P. Glutamate pathways of the beta-cell and the control of insulin secretion. Diabetes Res. Clin. Pract. 2017, 131, 149-153. [CrossRef]

67. Takahashi, H.; Yokoi, N.; Seino, S. Glutamate as intracellular and extracellular signals in pancreatic islet functions. Proc. Jpn. Acad. Ser. BPhys. Biol. Sci. 2019, 95, 246-260. [CrossRef]

68. Hoang, D.T.; Hara, M.; Jo, J. Design Principles of Pancreatic Islets: Glucose-Dependent Coordination of Hormone Pulses. PLoS ONE 2016, 11, e0152446. [CrossRef]

69. Kalwat, M.A.; Cobb, M.H. Mechanisms of the amplifying pathway of insulin secretion in the $\beta$ cell. Pharmacol. Ther. 2017, 179, 17-30. [CrossRef]

70. Villard, O.; Brun, J.F.; Bories, L.; Molinari, N.; Benhamou, P.Y.; Berney, T.; Wojtusciszyn, A. The Second Phase of Insulin Secretion in Nondiabetic Islet-Grafted Recipients Is Altered and Can Predict Graft Outcome. J. Clin. Endocrinol. Metab. 2018, 103, 1310-1319. [CrossRef]

71. Henquin, J.C.; Dufrane, D.; Kerr-Conte, J.; Nenquin, M. Dynamics of glucose-induced insulin secretion in normal human islets. Am. J. Physiol. Endocrinol. Metab. 2015, 309, E640-E650. [CrossRef] [PubMed]

72. Gembal, M.; Detimary, P.; Gilon, P.; Gao, Z.Y.; Henquin, J.C. Mechanisms by which glucose can control insulin release independently from its action on adenosine triphosphate-sensitive K+ channels in mouse B cells. J. Clin. Investig. 1993, 91, 871-880. [CrossRef] [PubMed]

73. Komatsu, M.; Takei, M.; Ishii, H.; Sato, Y. Glucose-stimulated insulin secretion: A newer perspective. J. Diabetes Investig. 2013, 4, 511-516. [CrossRef]

74. Pedersen, M.G.; Tagliavini, A.; Henquin, J.C. Calcium signaling and secretory granule pool dynamics underlie biphasic insulin secretion and its amplification by glucose: Experiments and modeling. Am. J. Physiol. Endocrinol. Metab. 2019, 316, E475-E486. [CrossRef] [PubMed]

75. Rorsman, P.; Ashcroft, F.M. Pancreatic $\beta$-Cell Electrical Activity and Insulin Secretion: Of Mice and Men. Physiol. Rev. 2018, 98, 117-214. [CrossRef]

76. Daniel, S.; Noda, M.; Straub, S.G.; Sharp, G.W. Identification of the docked granule pool responsible for the first phase of glucose-stimulated insulin secretion. Diabetes 1999, 48, 1686-1690. [CrossRef]

77. Rorsman, P.; Renström, E. Insulin granule dynamics in pancreatic beta cells. Diabetologia 2003, 46, 1029-1045. [CrossRef]

78. Nagamatsu, S.; Ohara-Imaizumi, M.; Nakamichi, Y.; Kikuta, T.; Nishiwaki, C. Imaging docking and fusion of insulin granules induced by antidiabetes agents: Sulfonylurea and glinide drugs preferentially mediate the fusion of newcomer, but not previously docked, insulin granules. Diabetes 2006, 55, 2819-2825. [CrossRef]

79. Ohara-Imaizumi, M.; Fujiwara, T.; Nakamichi, Y.; Okamura, T.; Akimoto, Y.; Kawai, J.; Matsushima, S.; Kawakami, H.; Watanabe, T.; Akagawa, K.; et al. Imaging analysis reveals mechanistic differences between first- and second-phase insulin exocytosis. J. Cell Biol. 2007, 177, 695-705. [CrossRef]

80. Kalwat, M.A.; Thurmond, D.C. Signaling mechanisms of glucose-induced F-actin remodeling in pancreatic islet $\beta$ cells. Exp. Mol. Med. 2013, 45, e37. [CrossRef]

81. Mourad, N.I.; Nenquin, M.; Henquin, J.C. Metabolic amplifying pathway increases both phases of insulin secretion independently of beta-cell actin microfilaments. Am. J. Physiol. Cell Physiol. 2010, 299, C389-C398. [CrossRef] [PubMed]

82. Wang, Z.; Thurmond, D.C. Mechanisms of biphasic insulin-granule exocytosis-roles of the cytoskeleton, small GTPases and SNARE proteins. J. Cell Sci. 2009, 122, 893-903. [CrossRef] [PubMed]

83. Mourad, N.I.; Nenquin, M.; Henquin, J.C. cAMP-mediated and metabolic amplification of insulin secretion are distinct pathways sharing independence of $\beta$-cell microfilaments. Endocrinology 2012, 153, 4644-4654. [CrossRef] [PubMed]

84. Mourad, N.I.; Nenquin, M.; Henquin, J.C. Amplification of insulin secretion by acetylcholine or phorbol ester is independent of $\beta$-cell microfilaments and distinct from metabolic amplification. Mol. Cell. Endocrinol. 2013, 367, 11-20. [CrossRef]

85. Shibasaki, T.; Takahashi, H.; Miki, T.; Sunaga, Y.; Matsumura, K.; Yamanaka, M.; Zhang, C.; Tamamoto, A.; Satoh, T.; Miyazaki, J.; et al. Essential role of Epac2/Rap1 signaling in regulation of insulin granule dynamics by cAMP. Proc. Natl. Acad. Sci. USA 2007, 104, 19333-19338. [CrossRef]

86. Leguina-Ruzzi, A.; Vodičková, A.; Holendová, B.; Pavluch, V.; Tauber, J.; Engstová, H.; Dlasková, A.; Ježek, P. Glucose-Induced Expression of DAPIT in Pancreatic $\beta$-Cells. Biomolecules 2020, 10, 1026. [CrossRef]

87. Bränström, R.; Leibiger, I.B.; Leibiger, B.; Corkey, B.E.; Berggren, P.O.; Larsson, O. Long chain coenzyme A esters activate the pore-forming subunit (Kir6. 2) of the ATP-regulated potassium channel. J. Biol. Chem. 1998, 273, 31395-31400. [CrossRef] 
88. Bränström, R.; Corkey, B.E.; Berggren, P.O.; Larsson, O. Evidence for a unique long chain acyl-CoA ester binding site on the ATP-regulated potassium channel in mouse pancreatic beta cells. J. Biol. Chem. 1997, 272, 17390-17394. [CrossRef]

89. Gribble, F.M.; Proks, P.; Corkey, B.E.; Ashcroft, F.M. Mechanism of cloned ATP-sensitive potassium channel activation by oleoyl-CoA. J. Biol. Chem. 1998, 273, 26383-26387. [CrossRef]

90. Prentki, M.; Vischer, S.; Glennon, M.C.; Regazzi, R.; Deeney, J.T.; Corkey, B.E. Malonyl-CoA and long chain acyl-CoA esters as metabolic coupling factors in nutrient-induced insulin secretion. J. Biol. Chem. 1992, 267, 5802-5810. [CrossRef]

91. Yang, S.N.; Shi, Y.; Yang, G.; Li, Y.; Yu, J.; Berggren, P.O. Ionic mechanisms in pancreatic $\beta$ cell signaling. Cell. Mol. Life Sci. Cmls 2014, 71, 4149-4177. [CrossRef] [PubMed]

92. Drews, G.; Krippeit-Drews, P.; Düfer, M. Electrophysiology of Islet Cells. In Advances in Experimental Medicine and Biology; Islam, M., Ed.; Springer: Dordrecht, The Netherlands, 2010; Volume 654, pp. 115-163. [CrossRef]

93. Bennett, K.; James, C.; Hussain, K. Pancreatic $\beta$-cell KATP channels: Hypoglycaemia and hyperglycaemia. Rev. Endocr. Metab. Disord. 2010, 11, 157-163. [CrossRef] [PubMed]

94. Szollosi, A.; Nenquin, M.; Henquin, J. Pharmacological stimulation and inhibition of insulin secretion in mouse islets lacking ATP-sensitive K+ channels. Br. J. Pharmacol. 2010, 159, 669-677. [CrossRef]

95. Soty, M.; Visa, M.; Soriano, S.; del Carmen Carmona, M.; Nadal, Á.; Novials, A. Involvement of ATP-sensitive Potassium (KATP) Channels in the Loss of Beta-cell Function Induced by Human Islet Amyloid Polypeptide. J. Biol. Chem. 2011, 286, 40857-40866. [CrossRef]

96. Rorsman, P.; Braun, M.; Zhang, Q. Regulation of calcium in pancreatic $\alpha$ - and $\beta$-cells in health and disease. Cell Calcium 2012, 51, 300-308. [CrossRef]

97. MacDonald, P.E. Signal integration at the level of ion channel and exocytotic function in pancreatic $\beta$-cells. Am. J. Physiol. Endocrinol. Metab. 2011, 301, E1065-E1069. [CrossRef]

98. Zhang, Q.; Chibalina, M.V.; Bengtsson, M.; Groschner, L.N.; Ramracheya, R.; Rorsman, N.J.; Leiss, V.; Nassar, M.A.; Welling, A.; Gribble, F.M.; et al. Na+ current properties in islet $\alpha$ - and $\beta$-cells reflect cell-specific Scn3a and Scn9a expression. J. Physiol. 2014, 592, 4677-4696. [CrossRef]

99. Tarasov, A.I.; Semplici, F.; Li, D.; Rizzuto, R.; Ravier, M.A.; Gilon, P.; Rutter, G.A. Frequency-dependent mitochondrial Ca(2+) accumulation regulates ATP synthesis in pancreatic $\beta$ cells. Pflug. Arch. Eur. J. Physiol. 2013, 465, 543-554. [CrossRef] [PubMed]

100. Lewandowski, S.L.; Cardone, R.L.; Foster, H.R.; Ho, T.; Potapenko, E.; Poudel, C.; VanDeusen, H.R.; Sdao, S.M.; Alves, T.C.; Zhao, X.; et al. Pyruvate Kinase Controls Signal Strength in the Insulin Secretory Pathway. Cell Metab. 2020, 32, 736-750.e735. [CrossRef]

101. Rorsman, P.; Braun, M. Regulation of insulin secretion in human pancreatic islets. Annu. Rev. Physiol. 2013, 75, 155-179. [CrossRef] [PubMed]

102. Smith, P.A.; Ashcroft, F.M.; Rorsman, P. Simultaneous recordings of glucose dependent electrical activity and ATP-regulated $\mathrm{K}(+)$-currents in isolated mouse pancreatic beta-cells. Febs Lett. 1990, 261, 187-190. [CrossRef]

103. Tarasov, A.I.; Girard, C.A.; Ashcroft, F.M. ATP sensitivity of the ATP-sensitive K+ channel in intact and permeabilized pancreatic beta-cells. Diabetes 2006, 55, 2446-2454. [CrossRef] [PubMed]

104. Catterall, W.A. Structure and regulation of voltage-gated Ca2+ channels. Annu. Rev. Cell Dev. Biol. 2000, 16, 521-555. [CrossRef] [PubMed]

105. Schulla, V.; Renström, E.; Feil, R.; Feil, S.; Franklin, I.; Gjinovci, A.; Jing, X.J.; Laux, D.; Lundquist, I.; Magnuson, M.A.; et al. Impaired insulin secretion and glucose tolerance in beta cell-selective Ca(v)1.2 Ca2+ channel null mice. Embo J. 2003, 22, 3844-3854. [CrossRef] [PubMed]

106. Jing, X.; Li, D.Q.; Olofsson, C.S.; Salehi, A.; Surve, V.V.; Caballero, J.; Ivarsson, R.; Lundquist, I.; Pereverzev, A.; Schneider, T.; et al. CaV2.3 calcium channels control second-phase insulin release. J. Clin. Investig. 2005, 115, 146-154. [CrossRef] [PubMed]

107. Kanno, T.; Suga, S.; Wu, J.; Kimura, M.; Wakui, M. Intracellular cAMP potentiates voltage-dependent activation of L-type Ca2+ channels in rat islet beta-cells. Pflug. Arch. Eur. J. Physiol. 1998, 435, 578-580. [CrossRef]

108. Rorsman, P.; Eliasson, L.; Kanno, T.; Zhang, Q.; Gopel, S. Electrophysiology of pancreatic $\beta$-cells in intact mouse islets of Langerhans. Prog. Biophys. Mol. Biol. 2011, 107, 224-235. [CrossRef]

109. Best, L. Glucose-induced electrical activity in rat pancreatic beta-cells: Dependence on intracellular chloride concentration. J. Physiol. 2005, 568, 137-144. [CrossRef]

110. Stuhlmann, T.; Planells-Cases, R.; Jentsch, T.J. LRRC8/VRAC anion channels enhance $\beta$-cell glucose sensing and insulin secretion. Nat. Commun. 2018, 9, 1974. [CrossRef]

111. Colsoul, B.; Schraenen, A.; Lemaire, K.; Quintens, R.; Van Lommel, L.; Segal, A.; Owsianik, G.; Talavera, K.; Voets, T.; Margolskee, R.F.; et al. Loss of high-frequency glucose-induced $\mathrm{Ca} 2+$ oscillations in pancreatic islets correlates with impaired glucose tolerance in Trpm5-/- mice. Proc. Natl. Acad. Sci. USA 2010, 107, 5208-5213. [CrossRef]

112. Sumoza-Toledo, A.; Penner, R. TRPM2: A multifunctional ion channel for calcium signalling. J. Physiol. 2011, 589, 1515-1525. [CrossRef] [PubMed]

113. Masgrau, R.; Churchill, G.C.; Morgan, A.J.; Ashcroft, S.J.; Galione, A. NAADP: A new second messenger for glucose-induced Ca2+ responses in clonal pancreatic beta cells. Curr. Biol. 2003, 13, 247-251. [CrossRef] 
114. Ostapchenko, V.G.; Chen, M.; Guzman, M.S.; Xie, Y.F.; Lavine, N.; Fan, J.; Beraldo, F.H.; Martyn, A.C.; Belrose, J.C.; Mori, Y.; et al. The Transient Receptor Potential Melastatin 2 (TRPM2) Channel Contributes to $\beta$-Amyloid Oligomer-Related Neurotoxicity and Memory Impairment. J. Neurosci. Off. J. Soc. Neurosci. 2015, 35, 15157-15169. [CrossRef] [PubMed]

115. Miyanohara, J.; Kakae, M.; Nagayasu, K.; Nakagawa, T.; Mori, Y.; Arai, K.; Shirakawa, H.; Kaneko, S. TRPM2 Channel Aggravates CNS Inflammation and Cognitive Impairment via Activation of Microglia in Chronic Cerebral Hypoperfusion. J. Neurosci. Off. J. Soc. Neurosci. 2018, 38, 3520-3533. [CrossRef] [PubMed]

116. Macdonald, M.J.; Hasan, N.M.; Longacre, M.J. Studies with leucine, beta-hydroxybutyrate and ATP citrate lyase-deficient beta cells support the acetoacetate pathway of insulin secretion. Biochim. Et Biophys. Acta 2008, 1780, 966-972. [CrossRef] [PubMed]

117. Gilon, P.; Ravier, M.A.; Jonas, J.C.; Henquin, J.C. Control mechanisms of the oscillations of insulin secretion in vitro and in vivo. Diabetes 2002, 51 (Suppl. 1), S144-S151. [CrossRef]

118. Beauvois, M.C.; Merezak, C.; Jonas, J.C.; Ravier, M.A.; Henquin, J.C.; Gilon, P. Glucose-induced mixed [Ca2+]c oscillations in mouse beta-cells are controlled by the membrane potential and the SERCA3 Ca2+-ATPase of the endoplasmic reticulum. Am. J. Physiol. Cell Physiol. 2006, 290, C1503-C1511. [CrossRef]

119. Sabourin, J.; Allagnat, F. Store-operated Ca2+ entry: A key component of the insulin secretion machinery. J. Mol. Endocrinol. 2016, 57, F35-F39. [CrossRef]

120. Sabourin, J.; Le Gal, L.; Saurwein, L.; Haefliger, J.A.; Raddatz, E.; Allagnat, F. Store-operated Ca2+ Entry Mediated by Orai1 and TRPC1 Participates to Insulin Secretion in Rat $\beta$-Cells. J. Biol. Chem. 2015, 290, 30530-30539. [CrossRef]

121. Rorsman, P.; Trube, G. Calcium and delayed potassium currents in mouse pancreatic beta-cells under voltage-clamp conditions. J. Physiol. 1986, 374, 531-550. [CrossRef]

122. Düfer, M.; Gier, B.; Wolpers, D.; Krippeit-Drews, P.; Ruth, P.; Drews, G. Enhanced glucose tolerance by SK4 channel inhibition in pancreatic beta-cells. Diabetes 2009, 58, 1835-1843. [CrossRef] [PubMed]

123. Vierra, N.C.; Dadi, P.K.; Jeong, I.; Dickerson, M.; Powell, D.R.; Jacobson, D.A. Type 2 Diabetes-Associated K+ Channel TALK-1 Modulates $\beta$-Cell Electrical Excitability, Second-Phase Insulin Secretion, and Glucose Homeostasis. Diabetes 2015, 64, 3818-3828. [CrossRef] [PubMed]

124. Jacobson, D.A.; Kuznetsov, A.; Lopez, J.P.; Kash, S.; Ammälä, C.E.; Philipson, L.H. Kv2.1 ablation alters glucose-induced islet electrical activity, enhancing insulin secretion. Cell Metab. 2007, 6, 229-235. [CrossRef] [PubMed]

125. Rebelato, E.; Santos, L.R.; Carpinelli, A.R.; Rorsman, P.; Abdulkader, F. Short-term high glucose culture potentiates pancreatic beta cell function. Sci. Rep. 2018, 8, 13061. [CrossRef]

126. Miki, T.; Nagashima, K.; Tashiro, F.; Kotake, K.; Yoshitomi, H.; Tamamoto, A.; Gonoi, T.; Iwanaga, T.; Miyazaki, J.; Seino, S. Defective insulin secretion and enhanced insulin action in KATP channel-deficient mice. Proc. Natl. Acad. Sci. USA 1998, 95, 10402-10406. [CrossRef]

127. Ravier, M.A.; Nenquin, M.; Miki, T.; Seino, S.; Henquin, J.C. Glucose controls cytosolic Ca2+ and insulin secretion in mouse islets lacking adenosine triphosphate-sensitive $\mathrm{K}+$ channels owing to a knockout of the pore-forming subunit Kir6.2. Endocrinology 2009, 150, 33-45. [CrossRef]

128. Yang, Y.Y.; Long, R.K.; Ferrara, C.T.; Gitelman, S.E.; German, M.S.; Yang, S.B. A new familial form of a late-onset, persistent hyperinsulinemic hypoglycemia of infancy caused by a novel mutation in KCNJ11. Channels (AustinTex.) 2017, 11, 636-647. [CrossRef]

129. Nenquin, M.; Szollosi, A.; Aguilar-Bryan, L.; Bryan, J.; Henquin, J.C. Both triggering and amplifying pathways contribute to fuel-induced insulin secretion in the absence of sulfonylurea receptor-1 in pancreatic beta-cells. J. Biol. Chem. 2004, 279, 32316-32324. [CrossRef]

130. Seghers, V.; Nakazaki, M.; DeMayo, F.; Aguilar-Bryan, L.; Bryan, J. Sur1 knockout mice. A model for K(ATP) channel-independent regulation of insulin secretion. J. Biol. Chem. 2000, 275, 9270-9277. [CrossRef]

131. Nakazaki, M.; Crane, A.; Hu, M.; Seghers, V.; Ullrich, S.; Aguilar-Bryan, L.; Bryan, J. cAMP-activated protein kinase-independent potentiation of insulin secretion by cAMP is impaired in SUR1 null islets. Diabetes 2002, 51, 3440-3449. [CrossRef]

132. Kikuta, T.; Ohara-Imaizumi, M.; Nakazaki, M.; Nishiwaki, C.; Nakamichi, Y.; Tei, C.; Aguilar-Bryan, L.; Bryan, J.; Nagamatsu, S. Docking and fusion of insulin secretory granules in SUR1 knock out mouse beta-cells observed by total internal reflection fluorescence microscopy. Febs Lett. 2005, 579, 1602-1606. [CrossRef] [PubMed]

133. Li, N.; Wu, J.X.; Ding, D.; Cheng, J.; Gao, N.; Chen, L. Structure of a Pancreatic ATP-Sensitive Potassium Channel. Cell 2017, 168, 101-110.e110. [CrossRef] [PubMed]

134. Martin, G.M.; Yoshioka, C.; Rex, E.A.; Fay, J.F.; Xie, Q.; Whorton, M.R.; Chen, J.Z.; Shyng, S.L. Cryo-EM structure of the ATP-sensitive potassium channel illuminates mechanisms of assembly and gating. eLife 2017, 6. [CrossRef] [PubMed]

135. Mikhailov, M.V.; Campbell, J.D.; de Wet, H.; Shimomura, K.; Zadek, B.; Collins, R.F.; Sansom, M.S.; Ford, R.C.; Ashcroft, F.M. 3-D structural and functional characterization of the purified KATP channel complex Kir6.2-SUR1. Embo J. 2005, 24, $4166-4175$. [CrossRef]

136. Nichols, C.G. KATP channels as molecular sensors of cellular metabolism. Nature 2006, 440, 470-476. [CrossRef]

137. Yang, H.Q.; Martinez-Ortiz, W.; Hwang, J.; Fan, X.; Cardozo, T.J.; Coetzee, W.A. Palmitoylation of the K(ATP) channel Kir6.2 subunit promotes channel opening by regulating PIP(2) sensitivity. Proc. Natl. Acad. Sci. USA 2020, 117, 10593-10602. [CrossRef]

138. Shyng, S.; Ferrigni, T.; Nichols, C.G. Regulation of KATP channel activity by diazoxide and MgADP. Distinct functions of the two nucleotide binding folds of the sulfonylurea receptor. J. Gen. Physiol 1997, 110, 643-654. [CrossRef] 
139. Vedovato, N.; Rorsman, O.; Hennis, K.; Ashcroft, F.M.; Proks, P. Role of the C-terminus of SUR in the differential regulation of $\beta$-cell and cardiac K(ATP) channels by MgADP and metabolism. J. Physiol. 2018, 596, 6205-6217. [CrossRef]

140. Shyng, S.L.; Nichols, C.G. Membrane phospholipid control of nucleotide sensitivity of KATP channels. Science 1998, 282, 1138-1141. [CrossRef]

141. Baukrowitz, T.; Schulte, U.; Oliver, D.; Herlitze, S.; Krauter, T.; Tucker, S.J.; Ruppersberg, J.P.; Fakler, B. PIP2 and PIP as determinants for ATP inhibition of KATP channels. Science 1998, 282, 1141-1144. [CrossRef]

142. Lin, Y.F.; Jan, Y.N.; Jan, L.Y. Regulation of ATP-sensitive potassium channel function by protein kinase A-mediated phosphorylation in transfected HEK293 cells. Embo J. 2000, 19, 942-955. [CrossRef] [PubMed]

143. Béguin, P.; Nagashima, K.; Nishimura, M.; Gonoi, T.; Seino, S. PKA-mediated phosphorylation of the human K(ATP) channel: Separate roles of Kir6.2 and SUR1 subunit phosphorylation. Embo J. 1999, 18, 4722-4732. [CrossRef]

144. Kline, C.F.; Wright, P.J.; Koval, O.M.; Zmuda, E.J.; Johnson, B.L.; Anderson, M.E.; Hai, T.; Hund, T.J.; Mohler, P.J. $\beta I V-S p e c t r i n$ and CaMKII facilitate Kir6.2 regulation in pancreatic beta cells. Proc. Natl. Acad. Sci. USA 2013, 110, 17576-17581. [CrossRef] [PubMed]

145. Ashcroft, F.M.; Harrison, D.E.; Ashcroft, S.J. Glucose induces closure of single potassium channels in isolated rat pancreatic beta-cells. Nature 1984, 312, 446-448. [CrossRef] [PubMed]

146. Yasui, S.; Mawatari, K.; Morizumi, R.; Furukawa, H.; Shimohata, T.; Harada, N.; Takahashi, A.; Nakaya, Y. Hydrogen peroxide inhibits insulin-induced ATP-sensitive potassium channel activation independent of insulin signaling pathway in cultured vascular smooth muscle cells. J. Med. Investig. JMI 2012, 59, 36-44. [CrossRef]

147. Finol-Urdaneta, R.K.; Remedi, M.S.; Raasch, W.; Becker, S.; Clark, R.B.; Strüver, N.; Pavlov, E.; Nichols, C.G.; French, R.J.; Terlau, H. Block of Kv1.7 potassium currents increases glucose-stimulated insulin secretion. Embo Mol. Med. 2012, 4, 424-434. [CrossRef] [PubMed]

148. MacDonald, P.E.; Salapatek, A.M.; Wheeler, M.B. Temperature and redox state dependence of native Kv2.1 currents in rat pancreatic beta-cells. J. Physiol. 2003, 546, 647-653. [CrossRef]

149. Mittal, M.; Gu, X.Q.; Pak, O.; Pamenter, M.E.; Haag, D.; Fuchs, D.B.; Schermuly, R.T.; Ghofrani, H.A.; Brandes, R.P.; Seeger, W.; et al. Hypoxia induces Kv channel current inhibition by increased NADPH oxidase-derived reactive oxygen species. Free Radic. Biol. Med. 2012, 52, 1033-1042. [CrossRef]

150. Grupe, M.; Myers, G.; Penner, R.; Fleig, A. Activation of store-operated I(CRAC) by hydrogen peroxide. Cell Calcium 2010, 48, 1-9. [CrossRef]

151. Kashio, M.; Tominaga, M. Redox Signal-mediated Enhancement of the Temperature Sensitivity of Transient Receptor Potential Melastatin 2 (TRPM2) Elevates Glucose-induced Insulin Secretion from Pancreatic Islets. J. Biol. Chem. 2015, 290, 12435-12442. [CrossRef]

152. Llanos, P.; Contreras-Ferrat, A.; Barrientos, G.; Valencia, M.; Mears, D.; Hidalgo, C. Glucose-Dependent Insulin Secretion in Pancreatic $\beta$-Cell Islets from Male Rats Requires Ca2+ Release via ROS-Stimulated Ryanodine Receptors. PLoS ONE 2015, 10, e0129238. [CrossRef] [PubMed]

153. Hara, Y.; Wakamori, M.; Ishii, M.; Maeno, E.; Nishida, M.; Yoshida, T.; Yamada, H.; Shimizu, S.; Mori, E.; Kudoh, J.; et al. LTRPC2 Ca2+-permeable channel activated by changes in redox status confers susceptibility to cell death. Mol. Cell 2002, 9, 163-173. [CrossRef]

154. Yosida, M.; Dezaki, K.; Uchida, K.; Kodera, S.; Lam, N.V.; Ito, K.; Rita, R.S.; Yamada, H.; Shimomura, K.; Ishikawa, S.E.; et al. Involvement of cAMP/EPAC/TRPM2 activation in glucose- and incretin-induced insulin secretion. Diabetes 2014, 63, 3394-3403. [CrossRef] [PubMed]

155. Xiao, H.; Jedrychowski, M.P.; Schweppe, D.K.; Huttlin, E.L.; Yu, Q.; Heppner, D.E.; Li, J.; Long, J.; Mills, E.L.; Szpyt, J.; et al. A Quantitative Tissue-Specific Landscape of Protein Redox Regulation during Aging. Cell 2020, 180, 968-983.e924. [CrossRef]

156. Paulsen, C.E.; Carroll, K.S. Cysteine-mediated redox signaling: Chemistry, biology, and tools for discovery. Chem. Rev. 2013, 113, 4633-4679. [CrossRef]

157. Huang, Y.; Roth, B.; Lü, W.; Du, J. Ligand recognition and gating mechanism through three ligand-binding sites of human TRPM2 channel. eLife 2019, 8. [CrossRef]

158. Kahancová, A.; Sklenář, F.; Ježek, P.; Dlasková, A. Regulation of glucose-stimulated insulin secretion by ATPase Inhibitory Factor 1 (IF1). Febs Lett. 2018, 592, 999-1009. [CrossRef]

159. Kahancová, A.; Sklenář, F.; Ježek, P.; Dlasková, A. Overexpression of native IF1 downregulates glucose-stimulated insulin secretion by pancreatic INS-1E cells. Sci. Rep. 2020, 10, 1551. [CrossRef]

160. Gu, J.; Zhang, L.; Zong, S.; Guo, R.; Liu, T.; Yi, J.; Wang, P.; Zhuo, W.; Yang, M. Cryo-EM structure of the mammalian ATP synthase tetramer bound with inhibitory protein IF1. Science 2019, 364, 1068-1075. [CrossRef]

161. Gledhill, J.R.; Montgomery, M.G.; Leslie, A.G.; Walker, J.E. How the regulatory protein, IF(1), inhibits F(1)-ATPase from bovine mitochondria. Proc. Natl. Acad. Sci. USA 2007, 104, 15671-15676. [CrossRef]

162. Esparza-Moltó, P.B.; Cuezva, J.M. Reprogramming Oxidative Phosphorylation in Cancer: A Role for RNA-Binding Proteins. Antioxid. Redox Signal. 2020. [CrossRef]

163. Shen, L.; Zhi, L.; Hu, W.; Wu, M.X. IEX-1 targets mitochondrial F1Fo-ATPase inhibitor for degradation. Cell Death Differ. 2009, 16, 603-612. [CrossRef] [PubMed] 
164. García-Aguilar, A.; Cuezva, J.M. A Review of the Inhibition of the Mitochondrial ATP Synthase by IF1 in vivo: Reprogramming Energy Metabolism and Inducing Mitohormesis. Front. Physiol. 2018, 9, 1322. [CrossRef] [PubMed]

165. Dlaskova, A.; Spacek, T.; Engstova, H.; Spackova, J.; Schrofel, A.; Holendova, B.; Smolkova, K.; Plecita-Hlavata, L.; Jezek, P. Mitochondrial cristae narrowing upon higher 2-oxoglutarate load. Biochim. Et Biophys. Acta. Bioenerg. 2019, 1860, $659-678$. [CrossRef] [PubMed]

166. Georgiadou, E.; Haythorne, E.; Dickerson, M.T.; Lopez-Noriega, L.; Pullen, T.J.; da Silva Xavier, G.; Davis, S.P.X.; MartinezSanchez, A.; Semplici, F.; Rizzuto, R.; et al. The pore-forming subunit MCU of the mitochondrial Ca(2+) uniporter is required for normal glucose-stimulated insulin secretion in vitro and in vivo in mice. Diabetologia 2020, 63, 1368-1381. [CrossRef]

167. McCormack, J.G.; Halestrap, A.P.; Denton, R.M. Role of calcium ions in regulation of mammalian intramitochondrial metabolism. Physiol. Rev. 1990, 70, 391-425. [CrossRef]

168. Drews, G.; Bauer, C.; Edalat, A.; Düfer, M.; Krippeit-Drews, P. Evidence against a Ca(2+)-induced potentiation of dehydrogenase activity in pancreatic beta-cells. Pflug. Arch. Eur. J. Physiol. 2015, 467, 2389-2397. [CrossRef]

169. Rutter, G.A.; Pralong, W.F.; Wollheim, C.B. Regulation of mitochondrial glycerol-phosphate dehydrogenase by Ca2+ within electropermeabilized insulin-secreting cells (INS-1). Biochim. Et Biophys. Acta 1992, 1175, 107-113. [CrossRef]

170. Alam, M.R.; Groschner, L.N.; Parichatikanond, W.; Kuo, L.; Bondarenko, A.I.; Rost, R.; Waldeck-Weiermair, M.; Malli, R.; Graier, W.F. Mitochondrial Ca2+ uptake 1 (MICU1) and mitochondrial ca2+ uniporter (MCU) contribute to metabolism-secretion coupling in clonal pancreatic $\beta$-cells. J. Biol. Chem. 2012, 287, 34445-34454. [CrossRef]

171. McKenna, J.P.; Ha, J.; Merrins, M.J.; Satin, L.S.; Sherman, A.; Bertram, R. Ca2+ Effects on ATP Production and Consumption Have Regulatory Roles on Oscillatory Islet Activity. Biophys J. 2016, 110, 733-742. [CrossRef]

172. Tsuboi, T.; da Silva Xavier, G.; Holz, G.G.; Jouaville, L.S.; Thomas, A.P.; Rutter, G.A. Glucagon-like peptide-1 mobilizes intracellular Ca2+ and stimulates mitochondrial ATP synthesis in pancreatic MIN6 beta-cells. Biochem. J. 2003, 369, 287-299. [CrossRef] [PubMed]

173. Hodson, D.J.; Tarasov, A.I.; Gimeno Brias, S.; Mitchell, R.K.; Johnston, N.R.; Haghollahi, S.; Cane, M.C.; Bugliani, M.; Marchetti, P.; Bosco, D.; et al. Incretin-modulated beta cell energetics in intact islets of Langerhans. Mol. Endocrinol. (Baltim. Md.) 2014, 28, 860-871. [CrossRef] [PubMed]

174. De Stefani, D.; Raffaello, A.; Teardo, E.; Szabò, I.; Rizzuto, R. A forty-kilodalton protein of the inner membrane is the mitochondrial calcium uniporter. Nature 2011, 476, 336-340. [CrossRef] [PubMed]

175. De Marchi, U.; Galindo, A.N.; Thevenet, J.; Hermant, A.; Bermont, F.; Lassueur, S.; Domingo, J.S.; Kussmann, M.; Dayon, L.; Wiederkehr, A. Mitochondrial lysine deacetylation promotes energy metabolism and calcium signaling in insulin-secreting cells. Faseb J. Off. Publ. Fed. Am. Soc. Exp. Biol. 2019, 33, 4660-4674. [CrossRef]

176. Quan, X.; Nguyen, T.T.; Choi, S.K.; Xu, S.; Das, R.; Cha, S.K.; Kim, N.; Han, J.; Wiederkehr, A.; Wollheim, C.B.; et al. Essential role of mitochondrial $\mathrm{Ca} 2+$ uniporter in the generation of mitochondrial $\mathrm{pH}$ gradient and metabolism-secretion coupling in insulin-releasing cells. J. Biol. Chem. 2015, 290, 4086-4096. [CrossRef]

177. Kennedy, E.D.; Rizzuto, R.; Theler, J.M.; Pralong, W.F.; Bastianutto, C.; Pozzan, T.; Wollheim, C.B. Glucose-stimulated insulin secretion correlates with changes in mitochondrial and cytosolic Ca2+ in aequorin-expressing INS-1 cells. J. Clin. Investig. 1996, 98, 2524-2538. [CrossRef]

178. Tarasov, A.I.; Semplici, F.; Ravier, M.A.; Bellomo, E.A.; Pullen, T.J.; Gilon, P.; Sekler, I.; Rizzuto, R.; Rutter, G.A. The mitochondrial $\mathrm{Ca} 2+$ uniporter MCU is essential for glucose-induced ATP increases in pancreatic $\beta$-cells. PLoS ONE 2012, 7, e39722. [CrossRef]

179. Wiederkehr, A.; Szanda, G.; Akhmedov, D.; Mataki, C.; Heizmann, C.W.; Schoonjans, K.; Pozzan, T.; Spät, A.; Wollheim, C.B. Mitochondrial matrix calcium is an activating signal for hormone secretion. Cell Metab. 2011, 13, 601-611. [CrossRef]

180. Müller, T.D.; Finan, B.; Bloom, S.R.; D’Alessio, D.; Drucker, D.J.; Flatt, P.R.; Fritsche, A.; Gribble, F.; Grill, H.J.; Habener, J.F.; et al. Glucagon-like peptide 1 (GLP-1). Mol. Metab. 2019, 30, 72-130. [CrossRef]

181. Furman, B.; Ong, W.K.; Pyne, N.J. Cyclic AMP signaling in pancreatic islets. Adv. Exp. Med. Biol. 2010, 654, 281-304. [CrossRef]

182. Lefkimmiatis, K.; Zaccolo, M. cAMP signaling in subcellular compartments. Pharmacol. Ther. 2014, 143, 295-304. [CrossRef] [PubMed]

183. Berridge, M.J. The Inositol Trisphosphate/Calcium Signaling Pathway in Health and Disease. Physiol. Rev. 2016, 96, 1261-1296. [CrossRef] [PubMed]

184. Husted, A.S.; Trauelsen, M.; Rudenko, O.; Hjorth, S.A.; Schwartz, T.W. GPCR-Mediated Signaling of Metabolites. Cell Metab. 2017, 25, 777-796. [CrossRef] [PubMed]

185. Salloum, G.; Jaafar, L.; El-Sibai, M. Rho A and Rac1: Antagonists moving forward. Tissue Cell 2020, 65, 101364. [CrossRef] [PubMed]

186. Dalle, S.; Ravier, M.A.; Bertrand, G. Emerging roles for $\beta$-arrestin- 1 in the control of the pancreatic $\beta$-cell function and mass: New therapeutic strategies and consequences for drug screening. Cell. Signal. 2011, 23, 522-528. [CrossRef]

187. Chen, Y.; Cann, M.J.; Litvin, T.N.; Iourgenko, V.; Sinclair, M.L.; Levin, L.R.; Buck, J. Soluble adenylyl cyclase as an evolutionarily conserved bicarbonate sensor. Science 2000, 289, 625-628. [CrossRef]

188. Taylor, S.S.; Ilouz, R.; Zhang, P.; Kornev, A.P. Assembly of allosteric macromolecular switches: Lessons from PKA. Nat. Rev. Mol. Cell Biol. 2012, 13, 646-658. [CrossRef]

189. Zhang, F.; Zhang, L.; Qi, Y.; Xu, H. Mitochondrial cAMP signaling. Cell. Mol. Life Sci. Cmls 2016, 73, 4577-4590. [CrossRef] 
190. Ould Amer, Y.; Hebert-Chatelain, E. Mitochondrial cAMP-PKA signaling: What do we really know? Biochim. Et Biophys. Acta. Bioenerg. 2018, 1859, 868-877. [CrossRef]

191. Härndahl, L.; Jing, X.J.; Ivarsson, R.; Degerman, E.; Ahrén, B.; Manganiello, V.C.; Renström, E.; Holst, L.S. Important role of phosphodiesterase $3 \mathrm{~B}$ for the stimulatory action of cAMP on pancreatic beta-cell exocytosis and release of insulin. J. Biol. Chem. 2002, 277, 37446-37455. [CrossRef]

192. Bünemann, M.; Gerhardstein, B.L.; Gao, T.; Hosey, M.M. Functional regulation of L-type calcium channels via protein kinase A-mediated phosphorylation of the beta(2) subunit. J. Biol. Chem. 1999, 274, 33851-33854. [CrossRef] [PubMed]

193. MacDonald, P.E.; Wang, X.; Xia, F.; El-kholy, W.; Targonsky, E.D.; Tsushima, R.G.; Wheeler, M.B. Antagonism of rat beta-cell voltage-dependent $\mathrm{K}+$ currents by exendin 4 requires dual activation of the cAMP/protein kinase A and phosphatidylinositol 3-kinase signaling pathways. J. Biol. Chem. 2003, 278, 52446-52453. [CrossRef] [PubMed]

194. Song, W.J.; Seshadri, M.; Ashraf, U.; Mdluli, T.; Mondal, P.; Keil, M.; Azevedo, M.; Kirschner, L.S.; Stratakis, C.A.; Hussain, M.A. Snapin mediates incretin action and augments glucose-dependent insulin secretion. Cell Metab. 2011, 13, 308-319. [CrossRef] [PubMed]

195. Somanath, S.; Partridge, C.J.; Marshall, C.; Rowe, T.; Turner, M.D. Snapin mediates insulin secretory granule docking, but not trans-SNARE complex formation. Biochem. Biophys. Res. Commun. 2016, 473, 403-407. [CrossRef] [PubMed]

196. Holz, G.G. Epac: A new cAMP-binding protein in support of glucagon-like peptide-1 receptor-mediated signal transduction in the pancreatic beta-cell. Diabetes 2004, 53, 5-13. [CrossRef]

197. Kang, G.; Leech, C.A.; Chepurny, O.G.; Coetzee, W.A.; Holz, G.G. Role of the cAMP sensor Epac as a determinant of KATP channel ATP sensitivity in human pancreatic beta-cells and rat INS-1 cells. J. Physiol. 2008, 586, 1307-1319. [CrossRef]

198. de Rooij, J.; Zwartkruis, F.J.; Verheijen, M.H.; Cool, R.H.; Nijman, S.M.; Wittinghofer, A.; Bos, J.L. Epac is a Rap1 guaninenucleotide-exchange factor directly activated by cyclic AMP. Nature 1998, 396, 474-477. [CrossRef]

199. Gloerich, M.; Bos, J.L. Epac: Defining a new mechanism for cAMP action. Annu. Rev. Pharmacol. Toxicol. 2010, 50, 355-375. [CrossRef]

200. Holz, G.G.; Leech, C.A.; Heller, R.S.; Castonguay, M.; Habener, J.F. cAMP-dependent mobilization of intracellular Ca2+ stores by activation of ryanodine receptors in pancreatic beta-cells. A Ca2+ signaling system stimulated by the insulinotropic hormone glucagon-like peptide-1-(7-37). J. Biol. Chem. 1999, 274, 14147-14156. [CrossRef]

201. Gilon, P.; Chae, H.Y.; Rutter, G.A.; Ravier, M.A. Calcium signaling in pancreatic $\beta$-cells in health and in Type 2 diabetes. Cell Calcium 2014, 56, 340-361. [CrossRef]

202. Kang, G.; Chepurny, O.G.; Holz, G.G. cAMP-regulated guanine nucleotide exchange factor II (Epac2) mediates Ca2+-induced Ca2+ release in INS-1 pancreatic beta-cells. J. Physiol. 2001, 536, 375-385. [CrossRef] [PubMed]

203. Ozaki, N.; Shibasaki, T.; Kashima, Y.; Miki, T.; Takahashi, K.; Ueno, H.; Sunaga, Y.; Yano, H.; Matsuura, Y.; Iwanaga, T.; et al. cAMP-GEFII is a direct target of cAMP in regulated exocytosis. Nat. Cell Biol. 2000, 2, 805-811. [CrossRef] [PubMed]

204. Kashima, Y.; Miki, T.; Shibasaki, T.; Ozaki, N.; Miyazaki, M.; Yano, H.; Seino, S. Critical role of cAMP-GEFII-Rim2 complex in incretin-potentiated insulin secretion. J. Biol. Chem. 2001, 276, 46046-46053. [CrossRef]

205. Yasuda, T.; Shibasaki, T.; Minami, K.; Takahashi, H.; Mizoguchi, A.; Uriu, Y.; Numata, T.; Mori, Y.; Miyazaki, J.; Miki, T.; et al. Rim2alpha determines docking and priming states in insulin granule exocytosis. Cell Metab. 2010, 12, 117-129. [CrossRef] [PubMed]

206. Zhao, X.; León, I.R.; Bak, S.; Mogensen, M.; Wrzesinski, K.; Højlund, K.; Jensen, O.N. Phosphoproteome analysis of functional mitochondria isolated from resting human muscle reveals extensive phosphorylation of inner membrane protein complexes and enzymes. Mol. Cell. Proteom. Mcp 2011, 10, M110.000299. [CrossRef] [PubMed]

207. Grimsrud, P.A.; Carson, J.J.; Hebert, A.S.; Hubler, S.L.; Niemi, N.M.; Bailey, D.J.; Jochem, A.; Stapleton, D.S.; Keller, M.P.; Westphall, M.S.; et al. A quantitative map of the liver mitochondrial phosphoproteome reveals posttranslational control of ketogenesis. Cell Metab. 2012, 16, 672-683. [CrossRef]

208. De Rasmo, D.; Micelli, L.; Santeramo, A.; Signorile, A.; Lattanzio, P.; Papa, S. cAMP regulates the functional activity, coupling efficiency and structural organization of mammalian FOF1 ATP synthase. Biochim. Et Biophys. Acta 2016, 1857, 350-358. [CrossRef]

209. Acin-Perez, R.; Russwurm, M.; Günnewig, K.; Gertz, M.; Zoidl, G.; Ramos, L.; Buck, J.; Levin, L.R.; Rassow, J.; Manfredi, G.; et al. A phosphodiesterase 2A isoform localized to mitochondria regulates respiration. J. Biol. Chem. 2011, 286, 30423-30432. [CrossRef]

210. Zhang, F.; Qi, Y.; Zhou, K.; Zhang, G.; Linask, K.; Xu, H. The cAMP phosphodiesterase Prune localizes to the mitochondrial matrix and promotes mtDNA replication by stabilizing TFAM. Embo Rep. 2015, 16, 520-527. [CrossRef]

211. García-Bermúdez, J.; Sánchez-Aragó, M.; Soldevilla, B.; Del Arco, A.; Nuevo-Tapioles, C.; Cuezva, J.M. PKA Phosphorylates the ATPase Inhibitory Factor 1 and Inactivates Its Capacity to Bind and Inhibit the Mitochondrial H(+)-ATP Synthase. Cell Rep. 2015, 12, 2143-2155. [CrossRef]

212. DiPilato, L.M.; Cheng, X.; Zhang, J. Fluorescent indicators of cAMP and Epac activation reveal differential dynamics of cAMP signaling within discrete subcellular compartments. Proc. Natl. Acad. Sci. USA 2004, 101, 16513-16518. [CrossRef] [PubMed]

213. Di Benedetto, G.; Scalzotto, E.; Mongillo, M.; Pozzan, T. Mitochondrial $\mathrm{Ca}^{2+}$ uptake induces cyclic AMP generation in the matrix and modulates organelle ATP levels. Cell Metab. 2013, 17, 965-975. [CrossRef] [PubMed]

214. Lefkimmiatis, K.; Leronni, D.; Hofer, A.M. The inner and outer compartments of mitochondria are sites of distinct cAMP/PKA signaling dynamics. J. Cell Biol. 2013, 202, 453-462. [CrossRef] [PubMed] 
215. Agnes, R.S.; Jernigan, F.; Shell, J.R.; Sharma, V.; Lawrence, D.S. Suborganelle sensing of mitochondrial cAMP-dependent protein kinase activity. J. Am. Chem. Soc. 2010, 132, 6075-6080. [CrossRef]

216. Srinivasan, S.; Spear, J.; Chandran, K.; Joseph, J.; Kalyanaraman, B.; Avadhani, N.G. Oxidative stress induced mitochondrial protein kinase A mediates cytochrome c oxidase dysfunction. PLoS ONE 2013, 8, e77129. [CrossRef]

217. Rosca, M.; Minkler, P.; Hoppel, C.L. Cardiac mitochondria in heart failure: Normal cardiolipin profile and increased threonine phosphorylation of complex IV. Biochim. Et Biophys. Acta 2011, 1807, 1373-1382. [CrossRef]

218. Parkkila, A.K.; Scarim, A.L.; Parkkila, S.; Waheed, A.; Corbett, J.A.; Sly, W.S. Expression of carbonic anhydrase V in pancreatic beta cells suggests role for mitochondrial carbonic anhydrase in insulin secretion. J. Biol. Chem. 1998, 273, 24620-24623. [CrossRef]

219. Shigeto, M.; Ramracheya, R.; Tarasov, A.I.; Cha, C.Y.; Chibalina, M.V.; Hastoy, B.; Philippaert, K.; Reinbothe, T.; Rorsman, N.; Salehi, A.; et al. GLP-1 stimulates insulin secretion by PKC-dependent TRPM4 and TRPM5 activation. J. Clin. Investig. 2015, 125, 4714-4728. [CrossRef]

220. Barker, C.J.; Berggren, P.O. New horizons in cellular regulation by inositol polyphosphates: Insights from the pancreatic $\beta$-cell. Pharm. Rev. 2013, 65, 641-669. [CrossRef]

221. Warwar, N.; Efendic, S.; Ostenson, C.G.; Haber, E.P.; Cerasi, E.; Nesher, R. Dynamics of glucose-induced localization of PKC isoenzymes in pancreatic beta-cells: Diabetes-related changes in the GK rat. Diabetes 2006, 55, 590-599. [CrossRef]

222. Seed Ahmed, M.; Pelletier, J.; Leumann, H.; Gu, H.F.; Östenson, C.G. Expression of Protein Kinase C Isoforms in Pancreatic Islets and Liver of Male Goto-Kakizaki Rats, a Model of Type 2 Diabetes. PLoS ONE 2015, 10, e0135781. [CrossRef] [PubMed]

223. Wuttke, A.; Yu, Q.; Tengholm, A. Autocrine Signaling Underlies Fast Repetitive Plasma Membrane Translocation of Conventional and Novel Protein Kinase C Isoforms in $\beta$ Cells. J. Biol. Chem. 2016, 291, 14986-14995. [CrossRef]

224. Hashimoto, T.; Mogami, H.; Tsuriya, D.; Morita, H.; Sasaki, S.; Kumada, T.; Suzuki, Y.; Urano, T.; Oki, Y.; Suda, T. G-proteincoupled receptor 40 agonist GW9508 potentiates glucose-stimulated insulin secretion through activation of protein kinase C $\alpha$ and $\varepsilon$ in INS-1 cells. PLoS ONE 2019, 14, e0222179. [CrossRef] [PubMed]

225. Newton, A.C. Protein kinase C: Perfectly balanced. Crit. Rev. Biochem. Mol. Biol. 2018, 53, 208-230. [CrossRef] [PubMed]

226. Gallegos, L.L.; Kunkel, M.T.; Newton, A.C. Targeting protein kinase C activity reporter to discrete intracellular regions reveals spatiotemporal differences in agonist-dependent signaling. J. Biol. Chem. 2006, 281, 30947-30956. [CrossRef] [PubMed]

227. Santo-Domingo, J.; Chareyron, I.; Dayon, L.; Núñez Galindo, A.; Cominetti, O.; Pilar Giner Giménez, M.; De Marchi, U.; Canto, C.; Kussmann, M.; Wiederkehr, A. Coordinated activation of mitochondrial respiration and exocytosis mediated by PKC signaling in pancreatic $\beta$ cells. Faseb J. Off. Publ. Fed. Am. Soc. Exp. Biol. 2017, 31, 1028-1045. [CrossRef]

228. Antico Arciuch, V.G.; Alippe, Y.; Carreras, M.C.; Poderoso, J.J. Mitochondrial kinases in cell signaling: Facts and perspectives. Adv. Drug Deliv. Rev. 2009, 61, 1234-1249. [CrossRef]

229. Straub, S.G.; Shanmugam, G.; Sharp, G.W. Stimulation of insulin release by glucose is associated with an increase in the number of docked granules in the beta-cells of rat pancreatic islets. Diabetes 2004, 53, 3179-3183. [CrossRef]

230. Vakilian, M.; Tahamtani, Y.; Ghaedi, K. A review on insulin trafficking and exocytosis. Gene 2019, 706, 52-61. [CrossRef]

231. Hutton, J.C.; Penn, E.J.; Peshavaria, M. Low-molecular-weight constituents of isolated insulin-secretory granules. Bivalent cations, adenine nucleotides and inorganic phosphate. Biochem. J. 1983, 210, 297-305. [CrossRef]

232. Mitchell, K.J.; Lai, F.A.; Rutter, G.A. Ryanodine receptor type I and nicotinic acid adenine dinucleotide phosphate receptors mediate Ca2+ release from insulin-containing vesicles in living pancreatic beta-cells (MIN6). J. Biol. Chem. 2003, 278, 11057-11064. [CrossRef] [PubMed]

233. Itoh, N.; Okamoto, H. Translational control of proinsulin synthesis by glucose. Nature 1980, 283, 100-102. [CrossRef] [PubMed]

234. Dodson, G.; Steiner, D. The role of assembly in insulin's biosynthesis. Curr. Opin. Struct. Biol. 1998, 8, 189-194. [CrossRef]

235. Orci, L.; Halban, P.; Perrelet, A.; Amherdt, M.; Ravazzola, M.; Anderson, R.G. pH-independent and -dependent cleavage of proinsulin in the same secretory vesicle. J. Cell Biol. 1994, 126, 1149-1156. [CrossRef] [PubMed]

236. Li, Y.V. Zinc and insulin in pancreatic beta-cells. Endocrine 2014, 45, 178-189. [CrossRef]

237. Trogden, K.P.; Zhu, X.; Lee, J.S.; Wright, C.V.E.; Gu, G.; Kaverina, I. Regulation of Glucose-Dependent Golgi-Derived Microtubules by cAMP/EPAC2 Promotes Secretory Vesicle Biogenesis in Pancreatic $\beta$ Cells. Curr. Biol. 2019, 29, 2339-2350.e2335. [CrossRef]

238. Li, M.; Du, W.; Zhou, M.; Zheng, L.; Song, E.; Hou, J. Proteomic analysis of insulin secretory granules in INS-1 cells by protein correlation profiling. Biophys. Rep. 2018, 4, 329-338. [CrossRef]

239. Davidson, H.W.; Wenzlau, J.M.; O’Brien, R.M. Zinc transporter 8 (ZnT8) and $\beta$ cell function. Trends Endocrinol. Metab. Tem 2014, 25, 415-424. [CrossRef]

240. Geng, X.; Li, L.; Watkins, S.; Robbins, P.D.; Drain, P. The insulin secretory granule is the major site of K(ATP) channels of the endocrine pancreas. Diabetes 2003, 52,767-776. [CrossRef]

241. Geng, X.; Lou, H.; Wang, J.; Li, L.; Swanson, A.L.; Sun, M.; Beers-Stolz, D.; Watkins, S.; Perez, R.G.; Drain, P. $\alpha$-Synuclein binds the K(ATP) channel at insulin-secretory granules and inhibits insulin secretion. Am. J. Physiol. Endocrinol. Metab. 2011, 300, E276-E286. [CrossRef]

242. Colsoul, B.; Nilius, B.; Vennekens, R. Transient receptor potential (TRP) cation channels in diabetes. Curr. Top. Med. Chem. 2013, 13, 258-269. [CrossRef]

243. Mitchell, K.J.; Pinton, P.; Varadi, A.; Tacchetti, C.; Ainscow, E.K.; Pozzan, T.; Rizzuto, R.; Rutter, G.A. Dense core secretory vesicles revealed as a dynamic $\mathrm{Ca}(2+)$ store in neuroendocrine cells with a vesicle-associated membrane protein aequorin chimaera. J. Cell Biol. 2001, 155, 41-51. [CrossRef] 
244. Blondel, O.; Moody, M.M.; Depaoli, A.M.; Sharp, A.H.; Ross, C.A.; Swift, H.; Bell, G.I. Localization of inositol trisphosphate receptor subtype 3 to insulin and somatostatin secretory granules and regulation of expression in islets and insulinoma cells. Proc. Natl. Acad. Sci. USA 1994, 91, 7777-7781. [CrossRef]

245. Dai, F.F.; Bhattacharjee, A.; Liu, Y.; Batchuluun, B.; Zhang, M.; Wang, X.S.; Huang, X.; Luu, L.; Zhu, D.; Gaisano, H.; et al. A Novel GLP1 Receptor Interacting Protein ATP6ap2 Regulates Insulin Secretion in Pancreatic Beta Cells. J. Biol. Chem. 2015, 290, 25045-25061. [CrossRef]

246. Boland, B.B.; Rhodes, C.J.; Grimsby, J.S. The dynamic plasticity of insulin production in $\beta$-cells. Mol. Metab. 2017, 6, 958-973. [CrossRef]

247. Song, S.H.; McIntyre, S.S.; Shah, H.; Veldhuis, J.D.; Hayes, P.C.; Butler, P.C. Direct measurement of pulsatile insulin secretion from the portal vein in human subjects. J. Clin. Endocrinol. Metab. 2000, 85, 4491-4499. [CrossRef] [PubMed]

248. Kasai, K.; Fujita, T.; Gomi, H.; Izumi, T. Docking is not a prerequisite but a temporal constraint for fusion of secretory granules. Traffic (Cph. Den.) 2008, 9, 1191-1203. [CrossRef]

249. Lai, Y.; Choi, U.B.; Leitz, J.; Rhee, H.J.; Lee, C.; Altas, B.; Zhao, M.; Pfuetzner, R.A.; Wang, A.L.; Brose, N.; et al. Molecular Mechanisms of Synaptic Vesicle Priming by Munc13 and Munc18. Neuron 2017, 95, 591-607.e510. [CrossRef]

250. Rizo, J.; Xu, J. The Synaptic Vesicle Release Machinery. Annu. Rev. Biophys. 2015, 44, 339-367. [CrossRef] [PubMed]

251. Wang, S.; Choi, U.B.; Gong, J.; Yang, X.; Li, Y.; Wang, A.L.; Yang, X.; Brunger, A.T.; Ma, C. Conformational change of syntaxin linker region induced by Munc13s initiates SNARE complex formation in synaptic exocytosis. Embo J. 2017, 36, 816-829. [CrossRef] [PubMed]

252. Huang, C.; Walker, E.M.; Dadi, P.K.; Hu, R.; Xu, Y.; Zhang, W.; Sanavia, T.; Mun, J.; Liu, J.; Nair, G.G.; et al. Synaptotagmin 4 Regulates Pancreatic $\beta$ Cell Maturation by Modulating the $\mathrm{Ca}(2+)$ Sensitivity of Insulin Secretion Vesicles. Dev. Cell 2018, 45, 347-361.e345. [CrossRef]

253. Maechler, P.; Wollheim, C.B. Mitochondrial glutamate acts as a messenger in glucose-induced insulin exocytosis. Nature 1999, 402, 685-689. [CrossRef] [PubMed]

254. Нøу, M.; Maechler, P.; Efanov, A.M.; Wollheim, C.B.; Berggren, P.O.; Gromada, J. Increase in cellular glutamate levels stimulates exocytosis in pancreatic beta-cells. Febs Lett. 2002, 531, 199-203. [CrossRef]

255. Casimir, M.; Lasorsa, F.M.; Rubi, B.; Caille, D.; Palmieri, F.; Meda, P.; Maechler, P. Mitochondrial glutamate carrier GC1 as a newly identified player in the control of glucose-stimulated insulin secretion. J. Biol. Chem. 2009, 284, 25004-25014. [CrossRef]

256. MacDonald, M.J.; Fahien, L.A. Glutamate is not a messenger in insulin secretion. J. Biol. Chem. 2000, 275, 34025-34027. [CrossRef] [PubMed]

257. Bertrand, G.; Ishiyama, N.; Nenquin, M.; Ravier, M.A.; Henquin, J.C. The elevation of glutamate content and the amplification of insulin secretion in glucose-stimulated pancreatic islets are not causally related. J. Biol. Chem. 2002, 277, 32883-32891. [CrossRef]

258. Gheni, G.; Ogura, M.; Iwasaki, M.; Yokoi, N.; Minami, K.; Nakayama, Y.; Harada, K.; Hastoy, B.; Wu, X.; Takahashi, H.; et al. Glutamate acts as a key signal linking glucose metabolism to incretin/cAMP action to amplify insulin secretion. Cell Rep. 2014, 9, 661-673. [CrossRef]

259. Aspinwall, C.A.; Brooks, S.A.; Kennedy, R.T.; Lakey, J.R. Effects of intravesicular H+ and extracellular H+ and Zn2+ on insulin secretion in pancreatic beta cells. J. Biol. Chem. 1997, 272, 31308-31314. [CrossRef] [PubMed]

260. Gammelsaeter, R.; Coppola, T.; Marcaggi, P.; Storm-Mathisen, J.; Chaudhry, F.A.; Attwell, D.; Regazzi, R.; Gundersen, V. A role for glutamate transporters in the regulation of insulin secretion. PLoS ONE 2011, 6, e22960. [CrossRef] [PubMed]

261. Hashim, M.; Yokoi, N.; Takahashi, H.; Gheni, G.; Okechi, O.S.; Hayami, T.; Murao, N.; Hidaka, S.; Minami, K.; Mizoguchi, A.; et al. Inhibition of SNAT5 Induces Incretin-Responsive State From Incretin-Unresponsive State in Pancreatic $\beta$-Cells: Study of $\beta$-Cell Spheroid Clusters as a Model. Diabetes 2018, 67, 1795-1806. [CrossRef]

262. Elrick, H.; Stimmler, L.; Hlad, C.J., Jr.; Arai, Y. Plasma insulin response to oral and intravenous glucose administration. J. Clin. Endocrinol. Metab. 1964, 24, 1076-1082. [CrossRef] [PubMed]

263. Ebert, R.; Unger, H.; Creutzfeldt, W. Preservation of incretin activity after removal of gastric inhibitory polypeptide (GIP) from rat gut extracts by immunoadsorption. Diabetologia 1983, 24, 449-454. [CrossRef] [PubMed]

264. Scrocchi, L.A.; Brown, T.J.; MaClusky, N.; Brubaker, P.L.; Auerbach, A.B.; Joyner, A.L.; Drucker, D.J. Glucose intolerance but normal satiety in mice with a null mutation in the glucagon-like peptide 1 receptor gene. Nat. Med. 1996, 2, 1254-1258. [CrossRef] [PubMed]

265. Scrocchi, L.A.; Marshall, B.A.; Cook, S.M.; Brubaker, P.L.; Drucker, D.J. Identification of glucagon-like peptide 1 (GLP-1) actions essential for glucose homeostasis in mice with disruption of GLP-1 receptor signaling. Diabetes 1998, 47, 632-639. [CrossRef] [PubMed]

266. Moon, M.J.; Park, S.; Kim, D.K.; Cho, E.B.; Hwang, J.I.; Vaudry, H.; Seong, J.Y. Structural and molecular conservation of glucagon-like Peptide-1 and its receptor confers selective ligand-receptor interaction. Front. Endocrinol. 2012, 3, 141. [CrossRef] [PubMed]

267. Kuhre, R.E.; Wewer Albrechtsen, N.J.; Hartmann, B.; Deacon, C.F.; Holst, J.J. Measurement of the incretin hormones: Glucagon-like peptide-1 and glucose-dependent insulinotropic peptide. J. Diabetes Its Complicat. 2015, 29, 445-450. [CrossRef]

268. Teraoku, H.; Lenzen, S. Dynamics of Insulin Secretion from EndoC- $\beta$ H1 $\beta$-Cell Pseudoislets in Response to Glucose and Other Nutrient and Nonnutrient Secretagogues. J. Diabetes Res. 2017, 2017, 2309630. [CrossRef] 
269. Graaf, C.d.; Donnelly, D.; Wootten, D.; Lau, J.; Sexton, P.M.; Miller, L.J.; Ahn, J.-M.; Liao, J.; Fletcher, M.M.; Yang, D.; et al. Glucagon-Like Peptide-1 and Its Class B G Protein-Coupled Receptors: A Long March to Therapeutic Successes. Pharmacol. Rev. 2016, 68, 954-1013. [CrossRef]

270. Moran, B.M.; Abdel-Wahab, Y.H.; Flatt, P.R.; McKillop, A.M. Activation of GPR119 by fatty acid agonists augments insulin release from clonal $\beta$-cells and isolated pancreatic islets and improves glucose tolerance in mice. Biol. Chem. 2014, 395, 453-464 [CrossRef] [PubMed]

271. Drucker, D.J.; Philippe, J.; Mojsov, S.; Chick, W.L.; Habener, J.F. Glucagon-like peptide I stimulates insulin gene expression and increases cyclic AMP levels in a rat islet cell line. Proc. Natl. Acad. Sci. USA 1987, 84, 3434-3438. [CrossRef]

272. Weir, G.C.; Mojsov, S.; Hendrick, G.K.; Habener, J.F. Glucagonlike peptide I (7-37) actions on endocrine pancreas. Diabetes 1989, 38, 338-342. [CrossRef] [PubMed]

273. Hjøllund, K.R.; Deacon, C.F.; Holst, J.J. Dipeptidyl peptidase-4 inhibition increases portal concentrations of intact glucagon-like peptide-1 (GLP-1) to a greater extent than peripheral concentrations in anaesthetised pigs. Diabetologia 2011, 54, 2206-2208. [CrossRef] [PubMed]

274. Herrmann, C.; Göke, R.; Richter, G.; Fehmann, H.C.; Arnold, R.; Göke, B. Glucagon-like peptide-1 and glucose-dependent insulin-releasing polypeptide plasma levels in response to nutrients. Digestion 1995, 56, 117-126. [CrossRef] [PubMed]

275. Wootten, D.; Reynolds, C.A.; Smith, K.J.; Mobarec, J.C.; Koole, C.; Savage, E.E.; Pabreja, K.; Simms, J.; Sridhar, R.; Furness, S.G.B.; et al. The Extracellular Surface of the GLP-1 Receptor Is a Molecular Trigger for Biased Agonism. Cell 2016, 165, 1632-1643. [CrossRef]

276. Sonoda, N.; Imamura, T.; Yoshizaki, T.; Babendure, J.L.; Lu, J.C.; Olefsky, J.M. Beta-Arrestin-1 mediates glucagon-like peptide-1 signaling to insulin secretion in cultured pancreatic beta cells. Proc. Natl. Acad. Sci. USA 2008, 105, 6614-6619. [CrossRef]

277. Montrose-Rafizadeh, C.; Avdonin, P.; Garant, M.J.; Rodgers, B.D.; Kole, S.; Yang, H.; Levine, M.A.; Schwindinger, W.; Bernier, M. Pancreatic glucagon-like peptide-1 receptor couples to multiple $\mathrm{G}$ proteins and activates mitogen-activated protein kinase pathways in Chinese hamster ovary cells. Endocrinology 1999, 140, 1132-1140. [CrossRef]

278. Light, P.E.; Manning Fox, J.E.; Riedel, M.J.; Wheeler, M.B. Glucagon-like peptide-1 inhibits pancreatic ATP-sensitive potassium channels via a protein kinase A- and ADP-dependent mechanism. Mol. Endocrinol. (Baltim. Md.) 2002, 16, 2135-2144. [CrossRef]

279. Kang, G.; Joseph, J.W.; Chepurny, O.G.; Monaco, M.; Wheeler, M.B.; Bos, J.L.; Schwede, F.; Genieser, H.G.; Holz, G.G. Epacselective cAMP analog 8-pCPT-2'-O-Me-cAMP as a stimulus for Ca2+-induced Ca2+ release and exocytosis in pancreatic beta-cells. J. Biol. Chem. 2003, 278, 8279-8285. [CrossRef]

280. Thompson, A.; Kanamarlapudi, V. Agonist-induced internalisation of the glucagon-like peptide- 1 receptor is mediated by the Gaq pathway. Biochem. Pharm. 2015, 93, 72-84. [CrossRef]

281. MacDonald, P.E.; Salapatek, A.M.; Wheeler, M.B. Glucagon-like peptide-1 receptor activation antagonizes voltage-dependent repolarizing $\mathrm{K}(+)$ currents in beta-cells: A possible glucose-dependent insulinotropic mechanism. Diabetes 2002, 51 (Suppl. 3), S443-S447. [CrossRef]

282. Vierra, N.C.; Dickerson, M.T.; Philipson, L.H.; Jacobson, D.A. Simultaneous Real-Time Measurement of the $\beta$-Cell Membrane Potential and $\mathrm{Ca}(2+)$ Influx to Assess the Role of Potassium Channels on $\beta$-Cell Function. Methods Mol. Biol. (CliftonN.J.) 2018, 1684, 73-84. [CrossRef]

283. Fernandez, J.; Valdeolmillos, M. Glucose-dependent stimulatory effect of glucagon-like peptide 1(7-36) amide on the electrical activity of pancreatic beta-cells recorded in vivo. Diabetes 1999, 48, 754-757. [CrossRef] [PubMed]

284. Fernandez, J.; Valdeolmillos, M. Synchronous glucose-dependent $[\mathrm{Ca}(2+)](\mathrm{i})$ oscillations in mouse pancreatic islets of Langerhans recorded in vivo. Febs Lett. 2000, 477, 33-36. [CrossRef] [PubMed]

285. Liu, T.; Li, H.; Gounko, N.V.; Zhou, Z.; Xu, A.; Hong, W.; Han, W. Detection of insulin granule exocytosis by an electrophysiology method with high temporal resolution reveals enlarged insulin granule pool in BIG3-knockout mice. Am. J. Physiol. Endocrinol. Metab. 2014, 307, E611-E618. [CrossRef] [PubMed]

286. Dupre, J.; Ross, S.A.; Watson, D.; Brown, J.C. Stimulation of insulin secretion by gastric inhibitory polypeptide in man. J. Clin. Endocrinol. Metab. 1973, 37, 826-828. [CrossRef]

287. Hinke, S.A.; Pauly, R.P.; Ehses, J.; Kerridge, P.; Demuth, H.U.; McIntosh, C.H.; Pederson, R.A. Role of glucose in chronic desensitization of isolated rat islets and mouse insulinoma (betaTC-3) cells to glucose-dependent insulinotropic polypeptide. J. Endocrinol. 2000, 165, 281-291. [CrossRef] [PubMed]

288. Ehses, J.A.; Pelech, S.L.; Pederson, R.A.; McIntosh, C.H. Glucose-dependent insulinotropic polypeptide activates the RafMek1/2-ERK1/2 module via a cyclic AMP/cAMP-dependent protein kinase/Rap1-mediated pathway. J. Biol. Chem. 2002, 277, 37088-37097. [CrossRef]

289. McIntosh, C.H.; Widenmaier, S.; Kim, S.J. Glucose-dependent insulinotropic polypeptide signaling in pancreatic $\beta$-cells and adipocytes. J. Diabetes Investig. 2012, 3, 96-106. [CrossRef]

290. Jitrapakdee, S.; Wutthisathapornchai, A.; Wallace, J.C.; MacDonald, M.J. Regulation of insulin secretion: Role of mitochondrial signalling. Diabetologia 2010, 53, 1019-1032. [CrossRef]

291. Joseph, J.W.; Jensen, M.V.; Ilkayeva, O.; Palmieri, F.; Alárcon, C.; Rhodes, C.J.; Newgard, C.B. The Mitochondrial Citrate/Isocitrate Carrier Plays a Regulatory Role in Glucose-stimulated Insulin Secretion. J. Biol. Chem. 2006, 281, 35624-35632. [CrossRef] 
292. Odegaard, M.L.; Joseph, J.W.; Jensen, M.V.; Lu, D.; Ilkayeva, O.; Ronnebaum, S.M.; Becker, T.C.; Newgard, C.B. The Mitochondrial 2-Oxoglutarate Carrier Is Part of a Metabolic Pathway That Mediates Glucose- and Glutamine-stimulated Insulin Secretion. J. Biol. Chem. 2010, 285, 16530-16537. [CrossRef] [PubMed]

293. Ronnebaum, S.M.; Ilkayeva, O.; Burgess, S.C.; Joseph, J.W.; Lu, D.; Stevens, R.D.; Becker, T.C.; Sherry, A.D.; Newgard, C.B.; Jensen, M.V. A Pyruvate Cycling Pathway Involving Cytosolic NADP-dependent Isocitrate Dehydrogenase Regulates Glucosestimulated Insulin Secretion. J. Biol. Chem. 2006, 281, 30593-30602. [CrossRef] [PubMed]

294. Lu, D.; Mulder, H.; Zhao, P.; Burgess, S.C.; Jensen, M.V.; Kamzolova, S.; Newgard, C.B.; Sherry, A.D. 13C NMR isotopomer analysis reveals a connection between pyruvate cycling and glucose-stimulated insulin secretion (GSIS). Proc. Natl. Acad. Sci. USA 2002, 99, 2708-2713. [CrossRef] [PubMed]

295. Guay, C.; Madiraju, S.R.; Aumais, A.; Joly, E.; Prentki, M. A role for ATP-citrate lyase, malic enzyme, and pyruvate/citrate cycling in glucose-induced insulin secretion. J. Biol. Chem. 2007, 282, 35657-35665. [CrossRef]

296. Pongratz, R.L.; Kibbey, R.G.; Shulman, G.I.; Cline, G.W. Cytosolic and mitochondrial malic enzyme isoforms differentially control insulin secretion. J. Biol. Chem. 2007, 282, 200-207. [CrossRef]

297. Farfari, S.; Schulz, V.; Corkey, B.; Prentki, M. Glucose-regulated anaplerosis and cataplerosis in pancreatic beta-cells: Possible implication of a pyruvate/citrate shuttle in insulin secretion. Diabetes 2000, 49, 718-726. [CrossRef]

298. El Azzouny, M.; Longacre, M.J.; Ansari, I.H.; Kennedy, R.T.; Burant, C.F.; MacDonald, M.J. Knockdown of ATP citrate lyase in pancreatic beta cells does not inhibit insulin secretion or glucose flux and implicates the acetoacetate pathway in insulin secretion. Mol. Metab. 2016, 5, 980-987. [CrossRef]

299. Chen, W.W.; Freinkman, E.; Wang, T.; Birsoy, K.; Sabatini, D.M. Absolute Quantification of Matrix Metabolites Reveals the Dynamics of Mitochondrial Metabolism. Cell 2016, 166, 1324-1337.e1311. [CrossRef]

300. Rydström, J. Mitochondrial NADPH, transhydrogenase and disease. Biochim. Et Biophys. Acta 2006, 1757, 721-726. [CrossRef]

301. Santos, L.R.B.; Muller, C.; de Souza, A.H.; Takahashi, H.K.; Spégel, P.; Sweet, I.R.; Chae, H.; Mulder, H.; Jonas, J.-C. NNT reverse mode of operation mediates glucose control of mitochondrial NADPH and glutathione redox state in mouse pancreatic $\beta$-cells. Mol. Metab. 2017, 6, 535-547. [CrossRef]

302. Freeman, H.C.; Hugill, A.; Dear, N.T.; Ashcroft, F.M.; Cox, R.D. Deletion of nicotinamide nucleotide transhydrogenase: A new quantitive trait locus accounting for glucose intolerance in C57BL/6J mice. Diabetes 2006, 55, 2153-2156. [CrossRef]

303. Toye, A.A.; Lippiat, J.D.; Proks, P.; Shimomura, K.; Bentley, L.; Hugill, A.; Mijat, V.; Goldsworthy, M.; Moir, L.; Haynes, A.; et al. A genetic and physiological study of impaired glucose homeostasis control in C57BL/6J mice. Diabetologia 2005, 48, 675-686. [CrossRef] [PubMed]

304. Freeman, H.; Shimomura, K.; Cox, R.D.; Ashcroft, F.M. Nicotinamide nucleotide transhydrogenase: A link between insulin secretion, glucose metabolism and oxidative stress. Biochem. Soc. Trans. 2006, 34, 806-810. [CrossRef] [PubMed]

305. Wong, N.; Blair, A.R.; Morahan, G.; Andrikopoulos, S. The deletion variant of nicotinamide nucleotide transhydrogenase (Nnt) does not affect insulin secretion or glucose tolerance. Endocrinology 2010, 151, 96-102. [CrossRef] [PubMed]

306. Hasan, N.M.; Longacre, M.J.; Stoker, S.W.; Kendrick, M.A.; MacDonald, M.J. Mitochondrial malic enzyme 3 is important for insulin secretion in pancreatic $\beta$-cells. Mol. Endocrinol. (Baltim. Md.) 2015, 29, 396-410. [CrossRef] [PubMed]

307. Spégel, P.; Mulder, H. Metabolomics Analysis of Nutrient Metabolism in $\beta$-Cells. J. Mol. Biol. 2020, 432, 1429-1445. [CrossRef]

308. El-Azzouny, M.; Evans, C.R.; Treutelaar, M.K.; Kennedy, R.T.; Burant, C.F. Increased glucose metabolism and glycerolipid formation by fatty acids and GPR40 receptor signaling underlies the fatty acid potentiation of insulin secretion. J. Biol. Chem. 2014, 289, 13575-13588. [CrossRef]

309. Ježek, J.; Dlasková, A.; Zelenka, J.; Jabůrek, M.; Ježek, P. H2O2-Activated Mitochondrial Phospholipase iPLA2 $\gamma$ Prevents Lipotoxic Oxidative Stress in Synergy with UCP2, Amplifies Signaling via G-Protein-Coupled Receptor GPR40, and Regulates Insulin Secretion in Pancreatic $\beta$-Cells. Antioxid. Redox Signal. 2015, 23, 958-972. [CrossRef]

310. Bränström, R.; Aspinwall, C.A.; Välimäki, S.; Ostensson, C.G.; Tibell, A.; Eckhard, M.; Brandhorst, H.; Corkey, B.E.; Berggren, P.O.; Larsson, O. Long-chain CoA esters activate human pancreatic beta-cell KATP channels: Potential role in Type 2 diabetes. Diabetologia 2004, 47, 277-283. [CrossRef]

311. Joseph, J.W.; Odegaard, M.L.; Ronnebaum, S.M.; Burgess, S.C.; Muehlbauer, J.; Sherry, A.D.; Newgard, C.B. Normal flux through ATP-citrate lyase or fatty acid synthase is not required for glucose-stimulated insulin secretion. J. Biol. Chem. 2007, 282, 31592-31600. [CrossRef]

312. Bender, K.; Maechler, P.; McClenaghan, N.H.; Flatt, P.R.; Newsholme, P. Overexpression of the malate-aspartate NADH shuttle member Aralar1 in the clonal beta-cell line BRIN-BD11 enhances amino-acid-stimulated insulin secretion and cell metabolism. Clin. Sci. 2009, 117, 321-330. [CrossRef] [PubMed]

313. Rubi, B.; del Arco, A.; Bartley, C.; Satrustegui, J.; Maechler, P. The malate-aspartate NADH shuttle member Aralar1 determines glucose metabolic fate, mitochondrial activity, and insulin secretion in beta cells. J. Biol. Chem. 2004, 279, 55659-55666. [CrossRef]

314. Newman, J.C.; Verdin, E. $\beta$-Hydroxybutyrate: A Signaling Metabolite. Annu. Rev. Nutr. 2017, 37, 51-76. [CrossRef] [PubMed]

315. Mitok, K.A.; Freiberger, E.C.; Schueler, K.L.; Rabaglia, M.E.; Stapleton, D.S.; Kwiecien, N.W.; Malec, P.A.; Hebert, A.S.; Broman, A.T.; Kennedy, R.T.; et al. Islet proteomics reveals genetic variation in dopamine production resulting in altered insulin secretion. J. Biol. Chem. 2018, 293, 5860-5877. [CrossRef] [PubMed] 
316. Abulizi, A.; Cardone, R.L.; Stark, R.; Lewandowski, S.L.; Zhao, X.; Hillion, J.; Ma, L.; Sehgal, R.; Alves, T.C.; Thomas, C.; et al. Multi-Tissue Acceleration of the Mitochondrial Phosphoenolpyruvate Cycle Improves Whole-Body Metabolic Health. Cell Metab. 2020, 32, 751-766.e711. [CrossRef]

317. Ashcroft, F.M.; Ashcroft, S.J.; Harrison, D.E. Effects of 2-ketoisocaproate on insulin release and single potassium channel activity in dispersed rat pancreatic beta-cells. J. Physiol. 1987, 385, 517-529. [CrossRef]

318. Panten, U.; Früh, E.; Reckers, K.; Rustenbeck, I. Acute metabolic amplification of insulin secretion in mouse islets: Role of cytosolic acetyl-CoA. Metab. Clin. Exp. 2016, 65, 1225-1229. [CrossRef]

319. Panten, U.; Willenborg, M.; Schumacher, K.; Hamada, A.; Ghaly, H.; Rustenbeck, I. Acute metabolic amplification of insulin secretion in mouse islets is mediated by mitochondrial export of metabolites, but not by mitochondrial energy generation. Metab. Clin. Exp. 2013, 62, 1375-1386. [CrossRef]

320. McClenaghan, N.H.; Flatt, P.R. Glucose and non-glucidic nutrients exert permissive effects on 2-keto acid regulation of pancreatic beta-cell function. Biochim. Et Biophys. Acta 1999, 1426, 110-118. [CrossRef]

321. Heissig, H.; Urban, K.A.; Hastedt, K.; Zünkler, B.J.; Panten, U. Mechanism of the insulin-releasing action of alpha-ketoisocaproate and related alpha-keto acid anions. Mol. Pharmacol. 2005, 68, 1097-1105. [CrossRef]

322. Gurgul-Convey, E.; Kaminski, M.T.; Lenzen, S. Physiological characterization of the human EndoC- $\beta \mathrm{H} 1 \beta$-cell line. Biochem. Biophys. Res. Commun. 2015, 464, 13-19. [CrossRef] [PubMed]

323. Bunik, V.I. Redox-Driven Signaling: 2-Oxo Acid Dehydrogenase Complexes as Sensors and Transmitters of Metabolic Imbalance. Antioxid. Redox Signal. 2019, 30, 1911-1947. [CrossRef] [PubMed]

324. Zhang, J.; Frerman, F.E.; Kim, J.J. Structure of electron transfer flavoprotein-ubiquinone oxidoreductase and electron transfer to the mitochondrial ubiquinone pool. Proc. Natl. Acad. Sci. USA 2006, 103, 16212-16217. [CrossRef] [PubMed]

325. Watmough, N.J.; Frerman, F.E. The electron transfer flavoprotein: Ubiquinone oxidoreductases. Biochim. Et Biophys. Acta 2010, 1797, 1910-1916. [CrossRef] [PubMed]

326. Husen, P.; Nielsen, C.; Martino, C.F.; Solov'yov, I.A. Molecular Oxygen Binding in the Mitochondrial Electron Transfer Flavoprotein. J. Chem. Inf. Modeling 2019, 59, 4868-4879. [CrossRef] [PubMed]

327. Brand, M.D. Mitochondrial generation of superoxide and hydrogen peroxide as the source of mitochondrial redox signaling. Free Radic. Biol. Med. 2016, 100, 14-31. [CrossRef]

328. Hull, J.; Hindy, M.E.; Kehoe, P.G.; Chalmers, K.; Love, S.; Conway, M.E. Distribution of the branched chain aminotransferase proteins in the human brain and their role in glutamate regulation. J. Neurochem. 2012, 123, 997-1009. [CrossRef]

329. Gao, Z.; Young, R.A.; Li, G.; Najafi, H.; Buettger, C.; Sukumvanich, S.S.; Wong, R.K.; Wolf, B.A.; Matschinsky, F.M. Distinguishing features of leucine and alpha-ketoisocaproate sensing in pancreatic beta-cells. Endocrinology 2003, 144, 1949-1957. [CrossRef]

330. Cheng, Q.; Beltran, V.D.; Chan, S.M.; Brown, J.R.; Bevington, A.; Herbert, T.P. System-L amino acid transporters play a key role in pancreatic $\beta$-cell signalling and function. J. Mol. Endocrinol. 2016, 56, 175-187. [CrossRef]

331. Giroix, M.H.; Saulnier, C.; Portha, B. Decreased pancreatic islet response to L-leucine in the spontaneously diabetic GK rat: Enzymatic, metabolic and secretory data. Diabetologia 1999, 42, 965-977. [CrossRef]

332. Denton, R.M. Regulation of mitochondrial dehydrogenases by calcium ions. Biochim. Et Biophys. Acta 2009, 1787, 1309-1316. [CrossRef]

333. Ævarsson, A.; Chuang, J.L.; Wynn, R.M.; Turley, S.; Chuang, D.T.; Hol, W.G. Crystal structure of human branched-chain alphaketoacid dehydrogenase and the molecular basis of multienzyme complex deficiency in maple syrup urine disease. Structure 2000, 8, 277-291. [CrossRef]

334. Noguchi, S.; Kondo, Y.; Ito, R.; Katayama, T.; Kazama, S.; Kadota, Y.; Kitaura, Y.; Harris, R.A.; Shimomura, Y. Ca(2+)-dependent inhibition of branched-chain $\alpha$-ketoacid dehydrogenase kinase by thiamine pyrophosphate. Biochem. Biophys. Res. Commun. 2018, 504, 916-920. [CrossRef] [PubMed]

335. Manders, R.J.; Little, J.P.; Forbes, S.C.; Candow, D.G. Insulinotropic and muscle protein synthetic effects of branched-chain amino acids: Potential therapy for type 2 diabetes and sarcopenia. Nutrients 2012, 4, 1664-1678. [CrossRef] [PubMed]

336. Yang, J.; Chi, Y.; Burkhardt, B.R.; Guan, Y.; Wolf, B.A. Leucine metabolism in regulation of insulin secretion from pancreatic beta cells. Nutr. Rev. 2010, 68, 270-279. [CrossRef]

337. Stein, D.T.; Stevenson, B.E.; Chester, M.W.; Basit, M.; Daniels, M.B.; Turley, S.D.; McGarry, J.D. The insulinotropic potency of fatty acids is influenced profoundly by their chain length and degree of saturation. J. Clin. Investig. 1997, 100, 398-403. [CrossRef] [PubMed]

338. Nyrén, R.; Chang, C.L.; Lindström, P.; Barmina, A.; Vorrsjö, E.; Ali, Y.; Juntti-Berggren, L.; Bensadoun, A.; Young, S.G.; Olivecrona, T.; et al. Localization of lipoprotein lipase and GPIHBP1 in mouse pancreas: Effects of diet and leptin deficiency. BMC Physiol. 2012, 12, 14. [CrossRef]

339. Cen, J.; Sargsyan, E.; Bergsten, P. Fatty acids stimulate insulin secretion from human pancreatic islets at fasting glucose concentrations via mitochondria-dependent and -independent mechanisms. Nutr. Metab. 2016, 13, 59. [CrossRef] [PubMed]

340. Fernandez, J.; Valdeolmillos, M. Increased levels of free fatty acids in fasted mice stimulate in vivo beta-cell electrical activity. Diabetes 1998, 47, 1707-1712. [CrossRef]

341. Frayn, K.N. Metabolic Regulation: A Human Perspective; John Wiley \& Sons: Hoboken, NJ, USA, 2009.

342. Ee, L.C.; Zheng, S.; Yao, L.; Tso, P. Lymphatic absorption of fatty acids and cholesterol in the neonatal rat. Am. J. Physiol. Gastrointest. Liver Physiol. 2000, 279, G325-G331. [CrossRef] 
343. Nauli, A.M.; Nassir, F.; Zheng, S.; Yang, Q.; Lo, C.M.; Vonlehmden, S.B.; Lee, D.; Jandacek, R.J.; Abumrad, N.A.; Tso, P. CD36 is important for chylomicron formation and secretion and may mediate cholesterol uptake in the proximal intestine. Gastroenterology 2006, 131, 1197-1207. [CrossRef]

344. Moss, C.E.; Glass, L.L.; Diakogiannaki, E.; Pais, R.; Lenaghan, C.; Smith, D.M.; Wedin, M.; Bohlooly, Y.M.; Gribble, F.M.; Reimann, F. Lipid derivatives activate GPR119 and trigger GLP-1 secretion in primary murine L-cells. Peptides 2016, 77, 16-20. [CrossRef] [PubMed]

345. Itoh, K.; Moriguchi, R.; Yamada, Y.; Fujita, M.; Yamato, T.; Oumi, M.; Holst, J.J.; Seino, Y. High saturated fatty acid intake induces insulin secretion by elevating gastric inhibitory polypeptide levels in healthy individuals. Nutr. Res. 2014, 34, 653-660. [CrossRef] [PubMed]

346. Winzell, M.S.; Ström, K.; Holm, C.; Ahrén, B. Glucose-stimulated insulin secretion correlates with beta-cell lipolysis. Nutr. Metab. Cardiovasc. Dis. Nmcd 2006, 16 (Suppl. 1), S11-S16. [CrossRef]

347. Cruz, W.S.; Kwon, G.; Marshall, C.A.; McDaniel, M.L.; Semenkovich, C.F. Glucose and insulin stimulate heparin-releasable lipoprotein lipase activity in mouse islets and INS-1 cells. A potential link between insulin resistance and beta-cell dysfunction. J. Biol. Chem. 2001, 276, 12162-12168. [CrossRef] [PubMed]

348. Marshall, B.A.; Tordjman, K.; Host, H.H.; Ensor, N.J.; Kwon, G.; Marshall, C.A.; Coleman, T.; McDaniel, M.L.; Semenkovich, C.F. Relative hypoglycemia and hyperinsulinemia in mice with heterozygous lipoprotein lipase (LPL) deficiency. Islet LPL regulates insulin secretion. J. Biol. Chem. 1999, 274, 27426-27432. [CrossRef] [PubMed]

349. Peyot, M.L.; Guay, C.; Latour, M.G.; Lamontagne, J.; Lussier, R.; Pineda, M.; Ruderman, N.B.; Haemmerle, G.; Zechner, R.; Joly, E.; et al. Adipose triglyceride lipase is implicated in fuel- and non-fuel-stimulated insulin secretion. J. Biol. Chem. 2009, 284, 16848-16859. [CrossRef]

350. Fujiwara, K.; Maekawa, F.; Yada, T. Oleic acid interacts with GPR40 to induce Ca2+ signaling in rat islet beta-cells: Mediation by PLC and L-type Ca2+ channel and link to insulin release. Am. J. Physiol. Endocrinol. Metab. 2005, 289, E670-E677. [CrossRef]

351. Khan, S.; Kowluru, A. CD36 mediates lipid accumulation in pancreatic beta cells under the duress of glucolipotoxic conditions: Novel roles of lysine deacetylases. Biochem. Biophys. Res. Commun. 2018, 495, 2221-2226. [CrossRef]

352. Veprik, A.; Laufer, D.; Weiss, S.; Rubins, N.; Walker, M.D. GPR41 modulates insulin secretion and gene expression in pancreatic $\beta$-cells and modifies metabolic homeostasis in fed and fasting states. Faseb J. Off. Publ. Fed. Am. Soc. Exp. Biol. 2016, 30, 3860-3869. [CrossRef]

353. Pujol, J.B.; Christinat, N.; Ratinaud, Y.; Savoia, C.; Mitchell, S.E.; Dioum, E.H.M. Coordination of GPR40 and Ketogenesis Signaling by Medium Chain Fatty Acids Regulates Beta Cell Function. Nutrients 2018, 10, 473. [CrossRef] [PubMed]

354. Moran, B.M.; Abdel-Wahab, Y.H.; Flatt, P.R.; McKillop, A.M. Evaluation of the insulin-releasing and glucose-lowering effects of GPR120 activation in pancreatic $\beta$-cells. DiabetesObes. Metab. 2014, 16, 1128-1139. [CrossRef]

355. Hauge, M.; Vestmar, M.A.; Husted, A.S.; Ekberg, J.P.; Wright, M.J.; Di Salvo, J.; Weinglass, A.B.; Engelstoft, M.S.; Madsen, A.N.; Luckmann, M.; et al. GPR40 (FFAR1)-Combined Gs and Gq signaling in vitro is associated with robust incretin secretagogue action ex vivo and in vivo. Mol. Metab. 2015, 4, 3-14. [CrossRef] [PubMed]

356. Mancini, A.D.; Bertrand, G.; Vivot, K.; Carpentier, É.; Tremblay, C.; Ghislain, J.; Bouvier, M.; Poitout, V. $\beta$-Arrestin Recruitment and Biased Agonism at Free Fatty Acid Receptor 1. J. Biol. Chem. 2015, 290, 21131-21140. [CrossRef] [PubMed]

357. Graciano, M.F.; Valle, M.M.; Curi, R.; Carpinelli, A.R. Evidence for the involvement of GPR40 and NADPH oxidase in palmitic acid-induced superoxide production and insulin secretion. Islets 2013, 5, 139-148. [CrossRef] [PubMed]

358. Sabrautzki, S.; Kaiser, G.; Przemeck, G.K.H.; Gerst, F.; Lorza-Gil, E.; Panse, M.; Sartorius, T.; Hoene, M.; Marschall, S.; Haring, H.U.; et al. Point mutation of Ffar1 abrogates fatty acid-dependent insulin secretion, but protects against HFD-induced glucose intolerance. Mol. Metab. 2017, 6, 1304-1312. [CrossRef]

359. Yamada, H.; Yoshida, M.; Ito, K.; Dezaki, K.; Yada, T.; Ishikawa, S.E.; Kakei, M. Potentiation of Glucose-stimulated Insulin Secretion by the GPR40-PLC-TRPC Pathway in Pancreatic $\beta$-Cells. Sci. Rep. 2016, 6, 25912. [CrossRef]

360. Qian, J.; Gu, Y.; Wu, C.; Yu, F.; Chen, Y.; Zhu, J.; Yao, X.; Bei, C.; Zhu, Q. Agonist-induced activation of human FFA1 receptor signals to extracellular signal-regulated kinase 1 and 2 through $\mathrm{Gq}$ - and Gi-coupled signaling cascades. Cell. Mol. Biol. Lett. 2017, 22, 13. [CrossRef]

361. Kristinsson, H.; Bergsten, P.; Sargsyan, E. Free fatty acid receptor 1 (FFAR1/GPR40) signaling affects insulin secretion by enhancing mitochondrial respiration during palmitate exposure. Biochim. Et Biophys. Acta 2015, 1853, 3248-3257. [CrossRef]

362. Tomita, T.; Hosoda, K.; Fujikura, J.; Inagaki, N.; Nakao, K. The G-Protein-Coupled Long-Chain Fatty Acid Receptor GPR40 and Glucose Metabolism. Front. Endocrinol. 2014, 5, 152. [CrossRef]

363. Vilas-Boas, E.A.; Karabacz, N.; Marsiglio-Librais, G.N.; Valle, M.M.R.; Nalbach, L.; Ampofo, E.; Morgan, B.; Carpinelli, A.R.; Roma, L.P. Chronic activation of GPR40 does not negatively impact upon BRIN-BD11 pancreatic $\beta$-cell physiology and function. Pharmacol. Rep. 2020, 72, 1725-1737. [CrossRef]

364. Bergeron, V.; Ghislain, J.; Poitout, V. The P21-activated kinase PAK4 is implicated in fatty-acid potentiation of insulin secretion downstream of free fatty acid receptor 1 . Islets 2016, 8, 157-164. [CrossRef]

365. Ferdaoussi, M.; Bergeron, V.; Zarrouki, B.; Kolic, J.; Cantley, J.; Fielitz, J.; Olson, E.N.; Prentki, M.; Biden, T.; MacDonald, P.E.; et al. G protein-coupled receptor (GPR)40-dependent potentiation of insulin secretion in mouse islets is mediated by protein kinase D1. Diabetologia 2012, 55, 2682-2692. [CrossRef] 
366. Ribas, G.S.; Vargas, C.R. Evidence that Oxidative Disbalance and Mitochondrial Dysfunction are Involved in the Pathophysiology of Fatty Acid Oxidation Disorders. Cell. Mol. Neurobiol. 2020. [CrossRef] [PubMed]

367. Nunes Marsiglio-Librais, G.; Aparecida Vilas-Boas, E.; Carlein, C.; Hoffmann, M.D.A.; Roma, L.P.; Carpinelli, A.R. Evidence for NADPH oxidase activation by GPR40 in pancreatic $\beta$-cells. Redox Rep. Commun. Free Radic. Res. 2020, 25, 41-50. [CrossRef]

368. Masiello, P.; Novelli, M.; Bombara, M.; Fierabracci, V.; Vittorini, S.; Prentki, M.; Bergamini, E. The antilipolytic agent 3,5dimethylpyrazole inhibits insulin release in response to both nutrient secretagogues and cyclic adenosine monophosphate agonists in isolated rat islets. Metab. Clin. Exp. 2002, 51, 110-114. [CrossRef] [PubMed]

369. Mulder, H.; Yang, S.; Winzell, M.S.; Holm, C.; Ahrén, B. Inhibition of lipase activity and lipolysis in rat islets reduces insulin secretion. Diabetes 2004, 53, 122-128. [CrossRef]

370. Fex, M.; Haemmerle, G.; Wierup, N.; Dekker-Nitert, M.; Rehn, M.; Ristow, M.; Zechner, R.; Sundler, F.; Holm, C.; Eliasson, L.; et al. A beta cell-specific knockout of hormone-sensitive lipase in mice results in hyperglycaemia and disruption of exocytosis. Diabetologia 2009, 52, 271-280. [CrossRef]

371. Mugabo, Y.; Zhao, S.; Seifried, A.; Gezzar, S.; Al-Mass, A.; Zhang, D.; Lamontagne, J.; Attane, C.; Poursharifi, P.; Iglesias, J.; et al Identification of a mammalian glycerol-3-phosphate phosphatase: Role in metabolism and signaling in pancreatic $\beta$-cells and hepatocytes. Proc. Natl. Acad. Sci. USA 2016, 113, E430-E439. [CrossRef]

372. Zhao, S.; Mugabo, Y.; Iglesias, J.; Xie, L.; Delghingaro-Augusto, V.; Lussier, R.; Peyot, M.L.; Joly, E.; Taib, B.; Davis, M.A.; et al. $\alpha / \beta$-Hydrolase domain-6-accessible monoacylglycerol controls glucose-stimulated insulin secretion. Cell Metab. 2014, 19, 993-1007. [CrossRef]

373. Mugabo, Y.; Zhao, S.; Lamontagne, J.; Al-Mass, A.; Peyot, M.L.; Corkey, B.E.; Joly, E.; Madiraju, S.R.M.; Prentki, M. Metabolic fate of glucose and candidate signaling and excess-fuel detoxification pathways in pancreatic $\beta$-cells. J. Biol. Chem. 2017, 292, 7407-7422. [CrossRef] [PubMed]

374. Guay, C.; Joly, E.; Pepin, E.; Barbeau, A.; Hentsch, L.; Pineda, M.; Madiraju, S.R.; Brunengraber, H.; Prentki, M. A role for cytosolic isocitrate dehydrogenase as a negative regulator of glucose signaling for insulin secretion in pancreatic B-cells. PLoS ONE 2013, 8, e77097. [CrossRef] [PubMed]

375. Jensen, M.D.; Nielsen, S. Insulin dose response analysis of free fatty acid kinetics. Metab. Clin. Exp. 2007, 56, 68-76. [CrossRef] [PubMed]

376. van der Vusse, G.J. Albumin as fatty acid transporter. Drug Metab. Pharmacokinet. 2009, 24, 300-307. [CrossRef]

377. Rossmeisl, M.; Flachs, P.; Brauner, P.; Sponarova, J.; Matejkova, O.; Prazak, T.; Ruzickova, J.; Bardova, K.; Kuda, O.; Kopecky, J. Role of energy charge and AMP-activated protein kinase in adipocytes in the control of body fat stores. Int. J. Obes. Relat. Metab. Disord. J. Int. Assoc. Study Obes. 2004, 28 (Suppl. 4), S38-S44. [CrossRef]

378. Thams, P.; Capito, K. L-arginine stimulation of glucose-induced insulin secretion through membrane depolarization and independent of nitric oxide. Eur. J. Endocrinol. 1999, 140, 87-93. [CrossRef] [PubMed]

379. Pi, M.; Wu, Y.; Lenchik, N.I.; Gerling, I.; Quarles, L.D. GPRC6A Mediates the Effects of 1-Arginine on Insulin Secretion in Mouse Pancreatic Islets. Endocrinology 2012, 153, 4608-4615. [CrossRef]

380. Gooding, J.R.; Jensen, M.V.; Dai, X.; Wenner, B.R.; Lu, D.; Arumugam, R.; Ferdaoussi, M.; MacDonald, P.E.; Newgard, C.B. Adenylosuccinate Is an Insulin Secretagogue Derived from Glucose-Induced Purine Metabolism. Cell Rep. 2015, 13, 157-167. [CrossRef]

381. Ferdaoussi, M.; Dai, X.; Jensen, M.V.; Wang, R.; Peterson, B.S.; Huang, C.; Ilkayeva, O.; Smith, N.; Miller, N.; Hajmrle, C.; et al. Isocitrate-to-SENP1 signaling amplifies insulin secretion and rescues dysfunctional $\beta$ cells. J. Clin. Investig. 2015, 125, 3847-3860. [CrossRef]

382. Stocker, S.; Van Laer, K.; Mijuskovic, A.; Dick, T.P. The Conundrum of Hydrogen Peroxide Signaling and the Emerging Role of Peroxiredoxins as Redox Relay Hubs. Antioxid. Redox Signal. 2018, 28, 558-573. [CrossRef]

383. Rhee, S.G.; Woo, H.A.; Kang, D. The Role of Peroxiredoxins in the Transduction of H2O2 Signals. Antioxid. Redox Signal. 2018, 28, 537-557. [CrossRef] [PubMed]

384. Sobotta, M.C.; Liou, W.; Stocker, S.; Talwar, D.; Oehler, M.; Ruppert, T.; Scharf, A.N.; Dick, T.P. Peroxiredoxin-2 and STAT3 form a redox relay for $\mathrm{H} 2 \mathrm{O} 2$ signaling. Nat. Chem. Biol. 2015, 11, 64-70. [CrossRef] [PubMed]

385. Jarvis, R.M.; Hughes, S.M.; Ledgerwood, E.C. Peroxiredoxin 1 functions as a signal peroxidase to receive, transduce, and transmit peroxide signals in mammalian cells. Free Radic. Biol. Med. 2012, 53, 1522-1530. [CrossRef] [PubMed]

386. Stancill, J.S.; Broniowska, K.A.; Oleson, B.J.; Naatz, A.; Corbett, J.A. Pancreatic beta-cells detoxify H2O2 through the peroxiredoxin/thioredoxin antioxidant system. J. Biol. Chem. 2019, 294, 4843-4853. [CrossRef]

387. Stancill, J.S.; Happ, J.T.; Broniowska, K.A.; Hogg, N.; Corbett, J.A. Peroxiredoxin 1 plays a primary role in protecting pancreatic $\beta$-cells from hydrogen peroxide and peroxynitrite. Am. J. Physiol. Regul. Integr. Comp. Physiol. 2020, 318, R1004-R1013. [CrossRef]

388. Grankvist, K.; Marklund, S.L.; Täljedal, I.B. CuZn-superoxide dismutase, Mn-superoxide dismutase, catalase and glutathione peroxidase in pancreatic islets and other tissues in the mouse. Biochem. J. 1981, 199, 393-398. [CrossRef] [PubMed]

389. Wood, Z.A.; Schröder, E.; Robin Harris, J.; Poole, L.B. Structure, mechanism and regulation of peroxiredoxins. Trends Biochem. Sci. 2003, 28, 32-40. [CrossRef]

390. Li, N.; Stojanovski, S.; Maechler, P. Mitochondrial hormesis in pancreatic $\beta$ cells: Does uncoupling protein 2 play a role? Oxidative Med. Cell. Longev. 2012, 2012, 740849. [CrossRef]

391. Sharma, K. Mitochondrial hormesis and diabetic complications. Diabetes 2015, 64, 663-672. [CrossRef] 
392. García-Martínez, B.I.; Ruiz-Ramos, M.; Pedraza-Chaverri, J.; Santiago-Osorio, E.; Mendoza-Núñez, V.M. Hypoglycemic Effect of Resveratrol: A Systematic Review and Meta-Analysis. Antioxidants 2021, 10, 69. [CrossRef]

393. Bagetta, D.; Maruca, A.; Lupia, A.; Mesiti, F.; Catalano, R.; Romeo, I.; Moraca, F.; Ambrosio, F.A.; Costa, G.; Artese, A.; et al. Mediterranean products as promising source of multi-target agents in the treatment of metabolic syndrome. Eur. J. Med. Chem. 2020, 186, 111903. [CrossRef] [PubMed]

394. Meng, J.M.; Cao, S.Y.; Wei, X.L.; Gan, R.Y.; Wang, Y.F.; Cai, S.X.; Xu, X.Y.; Zhang, P.Z.; Li, H.B. Effects and Mechanisms of Tea for the Prevention and Management of Diabetes Mellitus and Diabetic Complications: An Updated Review. Antioxidants 2019, 8, 170. [CrossRef]

395. Alkhatib, A.; Tsang, C.; Tuomilehto, J. Olive Oil Nutraceuticals in the Prevention and Management of Diabetes: From Molecules to Lifestyle. Int. J. Mol. Sci. 2018, 19, 2024. [CrossRef] [PubMed]

396. Roma, L.P.; Jonas, J.C. Nutrient Metabolism, Subcellular Redox State, and Oxidative Stress in Pancreatic Islets and beta-Cells. J. Mol. Biol. 2019. [CrossRef]

397. Forman, H.J.; Davies, K.J.; Ursini, F. How do nutritional antioxidants really work: Nucleophilic tone and para-hormesis versus free radical scavenging in vivo. Free Radic. Biol. Med. 2014, 66, 24-35. [CrossRef]

398. Ursini, F.; Maiorino, M.; Forman, H.J. Redox homeostasis: The Golden Mean of healthy living. Redox Biol. 2016, 8, 205-215. [CrossRef]

399. Smolková, K.; Mikó, E.; Kovács, T.; Leguina-Ruzzi, A.; Sipos, A.; Bai, P. Nuclear Factor Erythroid 2-Related Factor 2 in Regulating Cancer Metabolism. Antioxid. Redox Signal. 2020, 33, 966-997. [CrossRef]

400. Las, G.; Oliveira, M.F.; Shirihai, O.S. Emerging roles of $\beta$-cell mitochondria in type-2-diabetes. Mol. Asp. Med. 2020, 71, 100843. [CrossRef]

401. Mirabelli, M.; Russo, D.; Brunetti, A. The Role of Diet on Insulin Sensitivity. Nutrients 2020, 12, 3042. [CrossRef]

402. Cremonini, E.; Fraga, C.G.; Oteiza, P.I. (-)-Epicatechin in the control of glucose homeostasis: Involvement of redox-regulated mechanisms. Free Radic. Biol. Med. 2019, 130, 478-488. [CrossRef] 